\title{
The MiniBooNE Detector Technical Design Report
}

May 14, 2001

I. Stancu

University of Alabama, Tuscaloosa, AL 35487

S. Koutsoliotas

Bucknell University, Lewisburg, PA 17837

E. Church, G. J. VanDalen

University of California, Riverside, CA 92521

R. A. Johnson, J. L. Raaf, N. Suwonjandee

University of Cincinnati, Cincinnati, OH 45221

L. Bugel, J. M. Conrad,* B. T. Fleming, J. Formaggio,

M. H. Shaevitz, M. Sorel, E. D. Zimmerman

Columbia University, Nevis Labs, Irvington, NY 10533

D. Smith

Embry Riddle Aeronautical University, Prescott, AZ 86301

C. Bhat, S. J. Brice, B. C. Brown, R. Ford, F. G. Garcia, P. Kasper, T. Kobilarcik. I. Kourbanis, A. Malensek, W. Marsh, P. Martin, F. Mills, C. Moore, P. Nienaber, A. Russell, P. Spentzouris, R. Stefanski, T. Williams

Fermi National Accelerator Laboratory, Batavia, IL 60510

R. Tayloe

Indiana University, Bloomington, IN 47405

J. Boissevain, C. Espinoza, G. T. Garvey, E. Hawker, W. C. Louis,* G. B. Mills, V. Sandberg, B. Sapp, R. Schirato, T. N. Thompson, R. Van de Water, D. H. White

Los Alamos National Laboratory, Los Alamos, NM 87545

R. Imlay, A. Malik, W. Metcalf, M. Sung

Louisiana State University, Baton Rouge, LA 70803

B. P. Roe

University of Michigan, Ann Arbor, MI 48109

A. O. Bazarko, P. D. Meyers, W. Sands, F. C. Shoemaker

Princeton University, Princeton, NJ 08544

* Co-spokespersons: J. M. Conrad and W. C. Louis 


\section{Contents}

1 Executive Summary $\quad 9$

2 Overview of Detector Design $\quad 14$

2.1 Site Description . . . . . . . . . . . . . . . . . . . 14

2.2 Detector Elements . . . . . . . . . . . . . . . . . . 14

2.2 .1 Cylindrical Vault . . . . . . . . . . . . . . . 18

$2.2 .2 \quad$ Spherical Tank . . . . . . . . . . . . . . . . 18

2.2.3 Spherical Tank Internals . . . . . . . . . . . . . . . . 18

2.2.4 Detector Enclosure . . . . . . . . . . . . . . . . . . . . 19

2.3 Electronics and Data Acquisition . . . . . . . . . . . . 19

2.4 Calibration . . . . . . . . . . . . . . . 20

3 BooNE Detector Plant 21

3.1 MiniBooNE Detector Plant Site . . . . . . . . . . . . . . 23

3.1 .1 Location . . . . . . . . . . . . . . . . . . 23

3.1 .2 Soil Analysis . . . . . . . . . . . . . . . 23

3.1 .3 Access . . . . . . . . . . . . . . . 23

3.1.4 Utilities/Communications . . . . . . . . . . . . 24

3.2 Detector Containment Plant . . . . . . . . . . . . . . . . . 24

3.2 .1 Tank Vault . . . . . . . . . . . . . . . . 24

3.2.2 Oil Secondary Containment and Monitoring . . . . . . . . 26

3.2 .3 Tank . . . . . . . . . . . . . . . 26

3.2 .4 Access Portal . . . . . . . . . . . . . . 26

3.3 Support Plant . . . . . . . . . . . . . . . . . . . 30

3.3.1 Tank Access and Electronics Area . . . . . . . . . 30

3.3 .2 Utility Area . . . . . . . . . . . . . . . . . . 32

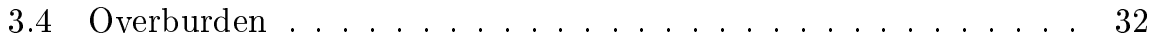

3.5 Additional Details . . . . . . . . . . . . . . 37

3.5.1 Heating/Cooling . . . . . . . . . . . . . 37

3.5 .2 Electrical . . . . . . . . . . . . . . . 37

3.5.3 Oil Plumbing . . . . . . . . . . . . . . . . . . 37

3.5.4 Signal Cables . . . . . . . . . . . . . . . . . 40 
3.5.5 Internal Component Installation . . . . . . . . . . . 40

3.5.6 Oil Transportation and Fill . . . . . . . . . . . . . . 40

4 Phototube Support Structure $\quad 41$

4.1 Design constraints . . . . . . . . . . . . . . . 41

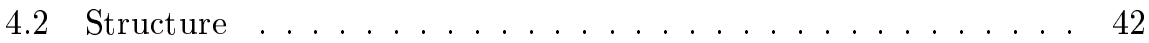

4.3 Surface finishes . . . . . . . . . . . . . . . . 48

4.4 Oil compatibility . . . . . . . . . . . . . . . . 54

4.5 In-tank cable plant . . . . . . . . . . . . . . . . 60

4.6 PMT preparation . . . . . . . . . . . . . . 62

4.7 Installation $\ldots \ldots \ldots \ldots \ldots \ldots \ldots \ldots \ldots \ldots$

5 Calibration Systems $\quad 66$

5.1 Introduction . . . . . . . . . . . . . . . 66

5.2 The Cosmic Ray Muon Calibration System . . . . . . . . . 66

5.3 Muon Tracker . . . . . . . . . . . . . . . . . . 67

5.4 Scintillation Cubes . . . . . . . . . . . . . . . 68

5.5 Laser Calibration System . . . . . . . . . . . . . . 72

6 Phototubes, Electronics, and Data Acquisition $\quad \mathbf{7 5}$

6.1 Introduction . . . . . . . . . . . . . . . . 75

6.2 Phototube Description . . . . . . . . . . . . . . . 75

6.3 Phototube Testing . . . . . . . . . . . . . . . . . . 85

6.4 Electronics Description . . . . . . . . . . . . . . . . 86

6.5 Electronics Upgrade . . . . . . . . . . . . . . . . . . . . 92

6.6 Beam Monitoring . . . . . . . . . . . . . . . . . . . . 95

6.7 DAQ Software . . . . . . . . . . . . . 96

6.8 Electronics, Signal Paths, and Physical Plant . . . . . . . . . 97

6.9 Appendix 6A: Photomultiplier Tube Bases and Preamplifier Elec-

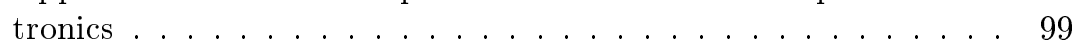

6.10 Appendix 6B: QT-Electronics . . . . . . . . . . . . . . 102

6.11 Appendix 6C: Commands and Data Structures for the QT and Trigger Cards . . . . . . . . . . . . . . . . . . 107 6.11 .1 RCVR Card Introduction . . . . . . . . . . . . 108

6.11 .2 RCVR Card Operation . . . . . . . . . . . . 108

6.11.3 RCVR Card Commands and Data Output Format . . . . 109

6.11 .4 QT Card Commands . . . . . . . . . . . . . . . 111

6.11 .5 Broadcast Card Introduction . . . . . . . . . . . . 114

6.11.6 Broadcast Card Commands and Data Formats . . . . . . 114

6.12 Appendix 6D: Communication Cable Timing Measurements . . . 116

6.12 .1 Introduction . . . . . . . . . . . . . . . 116

6.12 .2 Proposed Solution . . . . . . . . . . . . . . . 117

6.12 .3 Practical Realization . . . . . . . . . . . . . . . . 118

6.12 .4 Notes . . . . . . . . . . . . . . . . . . . 120 
7 Safety Considerations $\quad \mathbf{1 2 5}$

7.1 Fermilab ES\&H Requirements . . . . . . . . . . . . . . . . . . . . . . 125

7.2 Description of Hazards . . . . . . . . . . . . . . . . . 125

7.2 .1 Mechanical Hazards . . . . . . . . . . . . 126

7.2 .2 Electrical Hazards . . . . . . . . . . . . . . 126

7.2 .3 Fire Hazards . . . . . . . . . . . . . . . . . 126

7.2 .4 Laser Hazards . . . . . . . . . . . . . . . . . 126

7.3 Summary . . . . . . . . . . . . . . 126 


\section{List of Tables}

4.1 Layout of PMT's in the main tank. "Spacing" is the horizontal separation of the PMT's (measured from the centers of the globes) and can be compared to the row separation of $54.7 \mathrm{~cm}$. . 50

4.2 Layout of PMT's in the veto. The tubes are mounted in pairs. "Spacing" is the horizontal separation of the PMT's (measured from the centers of the clusters) and can be compared to the row separation of $187.6 \mathrm{~cm}$. . . . . . . . . . . . . . 51

4.3 Location and dimensions of the latitudinal hoops. Only the top hemisphere is shown; the bottom is a mirror image of the top. * The top lat doesn't actually exist: it is subsumed into the polar cap. . . . . . . . . . . . . . . . . . 5 51

4.4 Total weight, in tons, of various PSS components. . . . . . . . 52

5.1 Cube positions are listed in this table. The radius of the spiral is $60 \mathrm{~cm}$, the radius of the optical barrier is the tank radius $(610$ $\mathrm{cm})$ minus the veto $\operatorname{depth}(35 \mathrm{~cm})$. Therefore, $x=60 \sin (\phi)$, $y=610-35-$ depth,$z=60 \cos (\phi) . \ldots \ldots \ldots \ldots$

5.2 Laser bulb positions are listed in this table. $x=r \sin (\phi)$ and $z=r \cos (\phi) \ldots \ldots \ldots \ldots \ldots \ldots \ldots \ldots$ 


\section{List of Figures}

1.1 Sensitivity regions for the MiniBooNE experiment with $1 \times 10^{21}$ protons on target, or approximately two years of running. . . . . 10

1.2 Results of the fit for the case where $\Delta m^{2}=2 \mathrm{eV}^{2}$ and $\sin ^{2} 2 \theta=$ 0.002. The plot shows the $1 \sigma$ and $3 \sigma$ contours from the fit along with the LSND $90 \%$ and $99 \%$ regions. . . . . . . . . . . 11

1.3 A schematic drawing of the MiniBooNE detector. . . . . . . . . 12

2.1 The MiniBooNE site location. . . . . . . . . . . . . . 15

2.2 The MiniBooNE local site plan, showing the location of the 8 GeV Fixed Target Facility and the MiniBooNE Target Facility and Detector. . . . . . . . . . . . . . . 16

2.3 The MiniBooNE detector facility, consisting of the Detector Containment and Support Plant. . . . . . . . . . . . . 17

3.1 The MiniBooNE Detector Plant. Covering structures and earth overburden are not shown. . . . . . . . . . . . . . 22

3.2 The site plan for the MiniBooNE Detector Plant. The service road is shown as planned for the final configuration. FNAL project north is to the right. . . . . . . . . . 23

3.3 The Detector Containment Plant. . . . . . . . . . . . . . . 25

3.4 The detector tank in section. The support legs are not shown in this view. Dimensions are in inches. . . . . . . . . . . . 27

3.5 A perspective view of the detector tank access portal. . . . . . 28

3.6 Details of the detector tank access portal. Dimensions are in inches. 29

3.7 The MiniBooNE Support Plant in plan view showing the access, electronics, and utility areas. . . . . . . . . . . . 31

3.8 Elevation views of the Electronics Area showing the locations of the QT electronics racks, the power supply rack, the DAQ computer rack, the accelerator monitoring rack, and the calibration racks. . . . . . . . . . . . . . . . 33

3.9 A view of the utility area showing the location of the oil overflow tank, oil pumps, oil chiller/heat exchange unit, and other utility components. . . . . . . . . . . . . . . . 34 
3.10 A perspective view of the oil overflow tank. . . . . . . . 35

3.11 A schematic view of the MiniBooNE detector overburden. . . . . 36

3.12 A schematic figure illustrating the electrical service requirements in the MiniBooNE detector plant. . . . . . . . . . . . . 38

3.13 A schematic figure showing the oil plumbing scheme. . . . . . . 39

4.1 Main phototube layout. PMT's are not drawn to scale. There are a few gaps in this map where tubes had not yet been assigned. 43

4.2 Overview of the Phototube Support System. Note: this figure shows an obsolete version of the veto cluster. . . . . . . . . . 44

4.3 Struts with mounting hardware. The boss, welded to the tank wall, is on the left; the clamp for holding a lat section is on the right. . . . . . . . . . . . . . . . .

4.4 Survey of radial deviations of the tank from the best-fit sphere. Each main boss has been surveyed. In this cylindrical projection, the long lines are the edges of the panels forming the tank, the U-shaped lines are where the legs join the tank, and the squashed circle is the access port. . . . . . . . . . . 46

4.5 A typical panel of the optical barrier. . . . . . . . . . . 47

4.6 8-inch photomultiplier in LSND stand. . . . . . . . . . . 48

4.7 Veto PMT cluster. The strut mounts to a boss welded to the tank wall. The PMT's sit in the rings. . . . . . . . . . . . 49

4.8 The top polar cap. . . . . . . . . . . . . . . . . . 49

4.9 Albedo measurements for a variety of candidate white coatings. Also shown: the albedo curve published by Bicron for its paint (solid), and the phototube quantum efficiency in arbitrary units (dashed). . . . . . . . . . . . . . . . . .

4.10 Absorbance spectra of fresh mineral oil from the Witco Corporation (circles) and oil from the LSND storage tank (triangles). . . 55

4.11 Emission spectra of fresh mineral oil from the Witco Corporation (circles) and oil from the LSND storage tank (triangles). The samples have been excited at $280 \mathrm{~nm} . \ldots \ldots$. . . . . . .

4.12 Absorbance spectra for oil in which new samples of PVC-jacketed cable soaked. Top, black and white RG58 began to soak at room temperature on 25 Feb 2000 and the oil absorbance was measured on 3 March. Bottom, the same cables were put into fresh oil on 10 March and the oil absorbance was measured on 17 March. The solid line is the control spectrum for oil in which nothing soaked.

4.13 Absorbance spectra. Top, a sample of cable from LSND (i.e. a piece of cable that was immersed in the LSND tank during the duration of the LSND experiment) began to soak on 27 March 2000 and the oil absorbance was measured on 3 April. Bottom, oil absorbance measurements for oil samples exposed to teflonjacketed cables at $150^{\circ} \mathrm{F} . \ldots \ldots \ldots \ldots \ldots$ 
4.14 Cable feedthrough and mounting panel. . . . . . . . . . . . 61

5.1 Muon tracker .................... 68

5.2 PMT Base Configurations. . . . . . . . . . . . . . . . 69

5.3 Setup used to test a prototype cube with multiclad fiber. . . . . 71

5.4 Pulse height spectrum of through-going cosmic ray muons obtained with a prototype cube with a $1.5 \mathrm{~m}$ multiclad fiber. . . . . 71

5.5 MiniBooNE Calibration Systems. . . . . . . . . . . . . 74

6.1 Photograph of the Hamamatsu R1408(modified) 8 inch diameter, 9-dynode stage photomultiplier tube to be used in the MiniBooNE detector. It is shown in its wire-frame mounting fixture. . 77

6.2 An example of a summary of test results of a typical R1408 photomultiplier. ................... 78

6.3 Schematic diagram of the base used for the R1408 photomultiplier

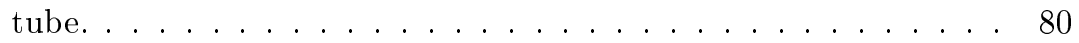

6.4 Schematic diagram of the base used for a 10 dynode stage version of the R5912 photomultiplier tube. . . . . . . . . . . . . 81

6.5 SPICE model of the photomultiplier tube dynode chain and anode circuitry. . . . . . . . . . . . . . . . . . . . . . 82

6.6 The preamplifer and high voltage circuit used with the photomultiplier tubes. .................... 83

6.7 Typical waveform of an anode pulse and its integral. . . . . . . . 84

6.8 Photomultiplier tube teststand schematic. . . . . . . . . . . 85

6.9 Charge and time data capture for a PMT pulse. The upper trace is the anode pulse and the second trace is the integrated charge. The "Discriminator" trace is a digital signal created when the anode pulse crosses a preset threshold. The "Vt" trace is the analog time ramp that starts with the "Discriminator" signal. The "Synchronous Discriminator" trace represents a signal that may be used by the trigger electronics. The $\mathrm{Vq}$ and $\mathrm{Vt}$ are analog signals that are digitized by FADCs at the sample times shown by the vertical lines, labeled by the clock ticks. . . . . . . . . .

6.10 Illustration of the MiniBooNE data acquisition system, showing the FADCs, circular buffer, and event building data streams. . . 88

6.11 The trigger hardware layout. . . . . . . . . . . . . . . 89

6.12 The trigger logic. . . . . . . . . . . . . . . . . 90 90

6.13 The module layout of a typical QT crate. . . . . . . . . . . 93

6.14 Layout of the MiniBooNE DAQ system. Shown are the arrangement of the 13 QT data acquisition crates and the Trigger crate. 94

6.15 Clean and dirty electrical power and cable layout for the MiniBooNE enclosure. . . . . . . . . . . . . . . . . . 97

6.16 A photograph inside of the LSND electronics hut, showing the rack layout of the QT crates and their power supplies. . . . . . . 98 
6.17 The symmetrical back termination design used for the R1408 base. This is compared with a more conventional connection to back terminate a base operated with a single cable. . . . . . . 100

6.18 A symmetric preamp-end cable termination that avoids the effect of the $\mathrm{RC}$ differenation due to the blocking capacitor used in a single cable system. . . . . . . . . . . . . . . . . . . 102

6.19 The front-end analog electronics for each PMT channel. The upper part of the schematic is the charge integrating circuit. It consists of a common base stage operating as a fast current sink connected to the integrating capacitor. The lower part shows the time interpolating circuit that starts a ramp on the leading edge of the discriminator pulse and resets the ramp on the second subsequent clock pulse. The discriminator threshold is typically set to fire for an input PMT pulse of $-7 \mathrm{mV}$. . . . . . . . . 104

6.20 The front-end digital electronics on a QT card. The FADCs feed the dual-ported memories, which in turn feed the FIFOs with selected time-stamp data. Shown at the bottom of the figure is the VME addres decoding and bus interface logic. . . . . . . . 105

6.21 The LSND RCVR Card used to communicate between the QT Crate and the Trigger Broadcast Card. It distributes TSA signals and stacks the broadcast timestamps. . . . . . . . . . 112

6.22 Plot of the SPICE simulation of the cable delay timing scheme. Voltages at the T1-T2 and T2-T3 junctions are shown on the traces marked with squares and diamonds, respectively. The plot is shown in landscape mode to allow better resolution of the waveform. . . . . . . . . . . . . . . . . 119

6.23 schematic of the SPICE simulation. . . . . . . . . . . 120

6.24 Illustration of the test setup. The setup used 320m of RG-223 coaxial cable and 100ns long termination cable stubs at either end.121

6.25 Tektronix 620 DSO trace at the driving point (T1-T2 junction). . 122

6.26 Tektronix 620 DSO trace at the pickoff point (T2-T3 junction). This is the signal that provides the timing and cable propagation delay information to the MiniBooNE DAQ system. . . . . . . . . 123 


\section{Chapter 1}

\section{Executive Summary}

The MiniBooNE experiment [1] is motivated by the LSND observation,[2] which has been interpreted as $\nu_{\mu} \rightarrow \nu_{e}$ oscillations, and by the atmospheric neutrino deficit, $[3,4,5]$ which may be ascribed to $\nu_{\mu}$ oscillations into another type of neutrino. MiniBooNE is a single-detector experiment designed to: obtain $\sim 1000$ $\nu_{\mu} \rightarrow \nu_{e}$ events if the LSND signal is due to $\nu_{\mu} \rightarrow \nu_{e}$ oscillations, establishing the oscillation signal at the $>5 \sigma$ level as shown in Fig. 1.1; extend the search for $\nu_{\mu} \rightarrow \nu_{e}$ oscillations significantly beyond what has been studied previously if no signal is observed; search for $\nu_{\mu}$ disappearance to address the atmospheric neutrino deficit with a signal that is a suppression of the rate of $\nu_{\mu} C \rightarrow \mu N$ events from the expected 600,000 per year; measure the oscillation parameters as shown in Fig. 1.2 if oscillations are observed; and test CP conservation in the lepton sector if oscillations are observed by running with separate $\nu_{\mu}$ and $\bar{\nu}_{\mu}$ beams.

The detector will consist of a spherical tank $6.1 \mathrm{~m}$ (20 feet) in radius, as shown in Fig. 1.3, that stands in a 45-foot diameter cylindrical vault. An inner tank structure at $5.75 \mathrm{~m}$ radius will support 1280 8-inch phototubes $(10 \%$ coverage) pointed inward and optically isolated from the outer region of the tank. The tank will be filled with $807 \mathrm{t}$ of mineral oil, resulting in a $445 \mathrm{t}$ fiducial volume. The outer tank volume will serve as a veto shield for identifying particles both entering and leaving the detector with 240 phototubes mounted on the tank wall. Above the detector tank will be an electronics enclosure that houses the fast electronics and data acquistion system and a utilities enclosure that houses the plumbing, overflow tank, and calibration laser. The detector will be located $\sim 550 \mathrm{~m}$ from the Booster neutrino source.

The neutrino beam, produced using $8 \mathrm{GeV}$ protons from the Booster at FNAL, will consist of a target within a focusing system, followed by a $\sim 50 \mathrm{~m}$ long pion decay volume. The low energy, high intensity and $1 \mu$ s time-structure of a neutrino beam produced from the Booster beam are ideal for this experiment. We assume that the Booster can reliably deliver protons for a typical run 


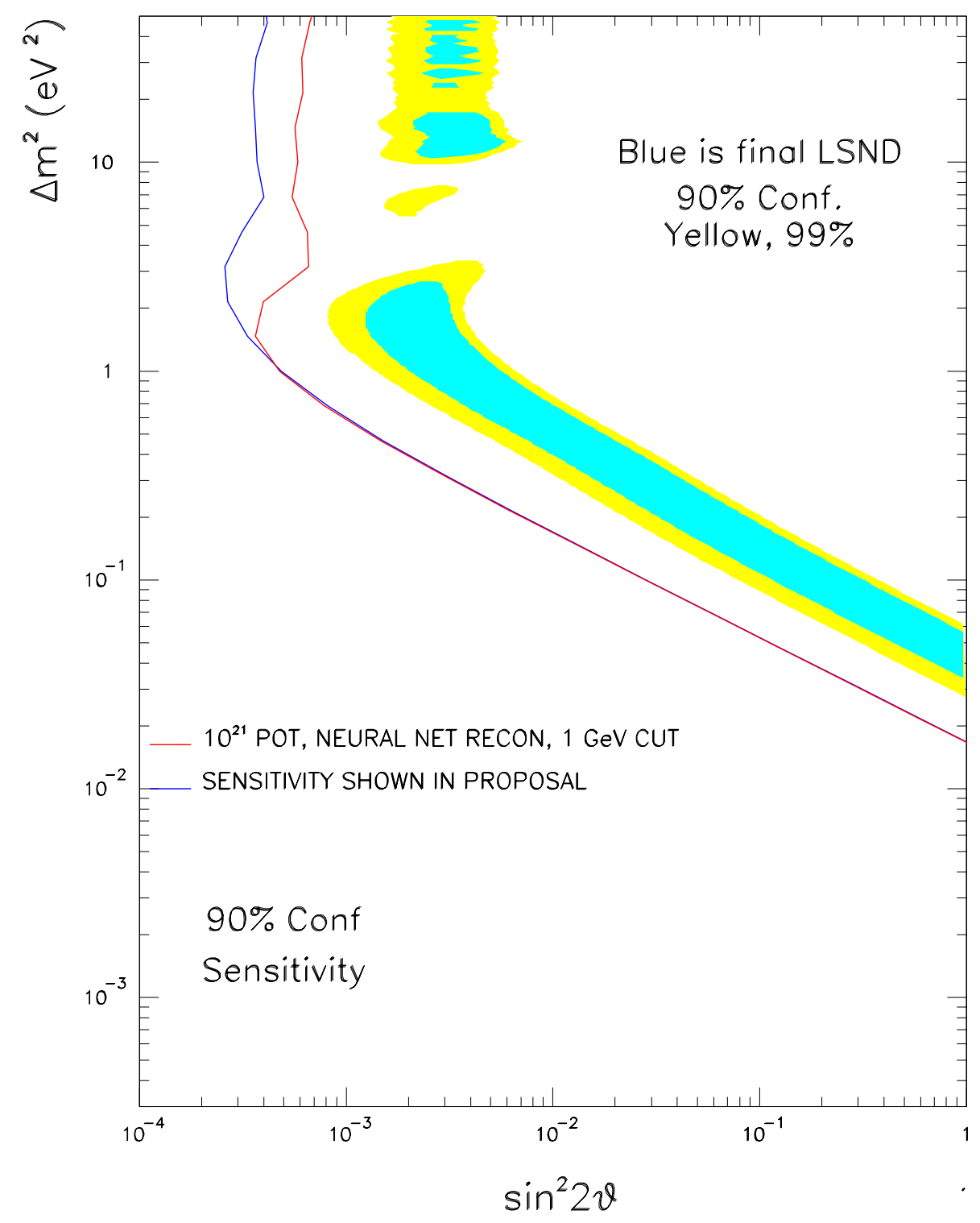

Figure 1.1: Sensitivity regions for the MiniBooNE experiment with $1 \times 10^{21}$ protons on target, or approximately two years of running. 


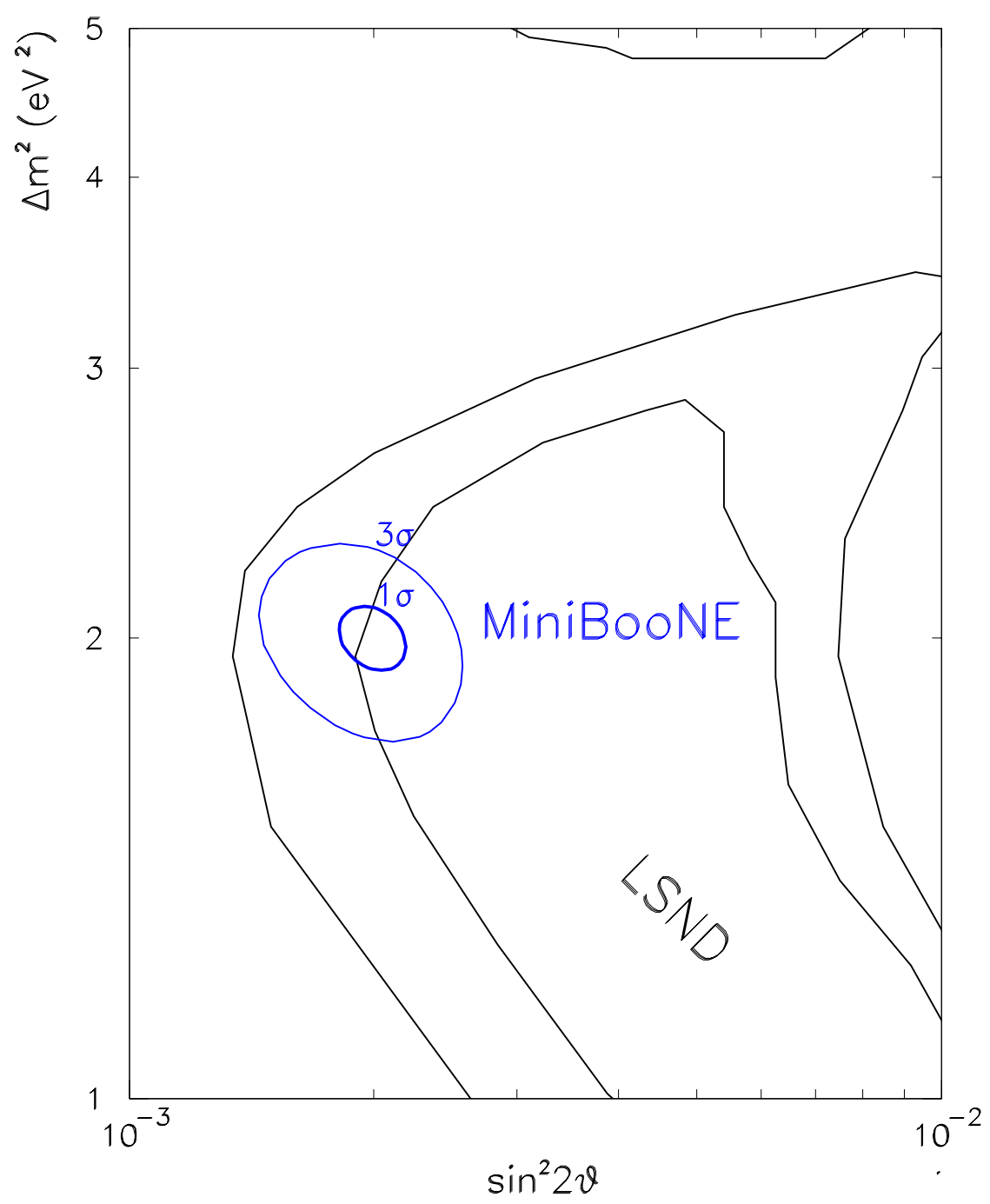

Figure 1.2: Results of the fit for the case where $\Delta m^{2}=2 \mathrm{eV}^{2}$ and $\sin ^{2} 2 \theta=$ 0.002. The plot shows the $1 \sigma$ and $3 \sigma$ contours from the fit along with the LSND $90 \%$ and $99 \%$ regions. 


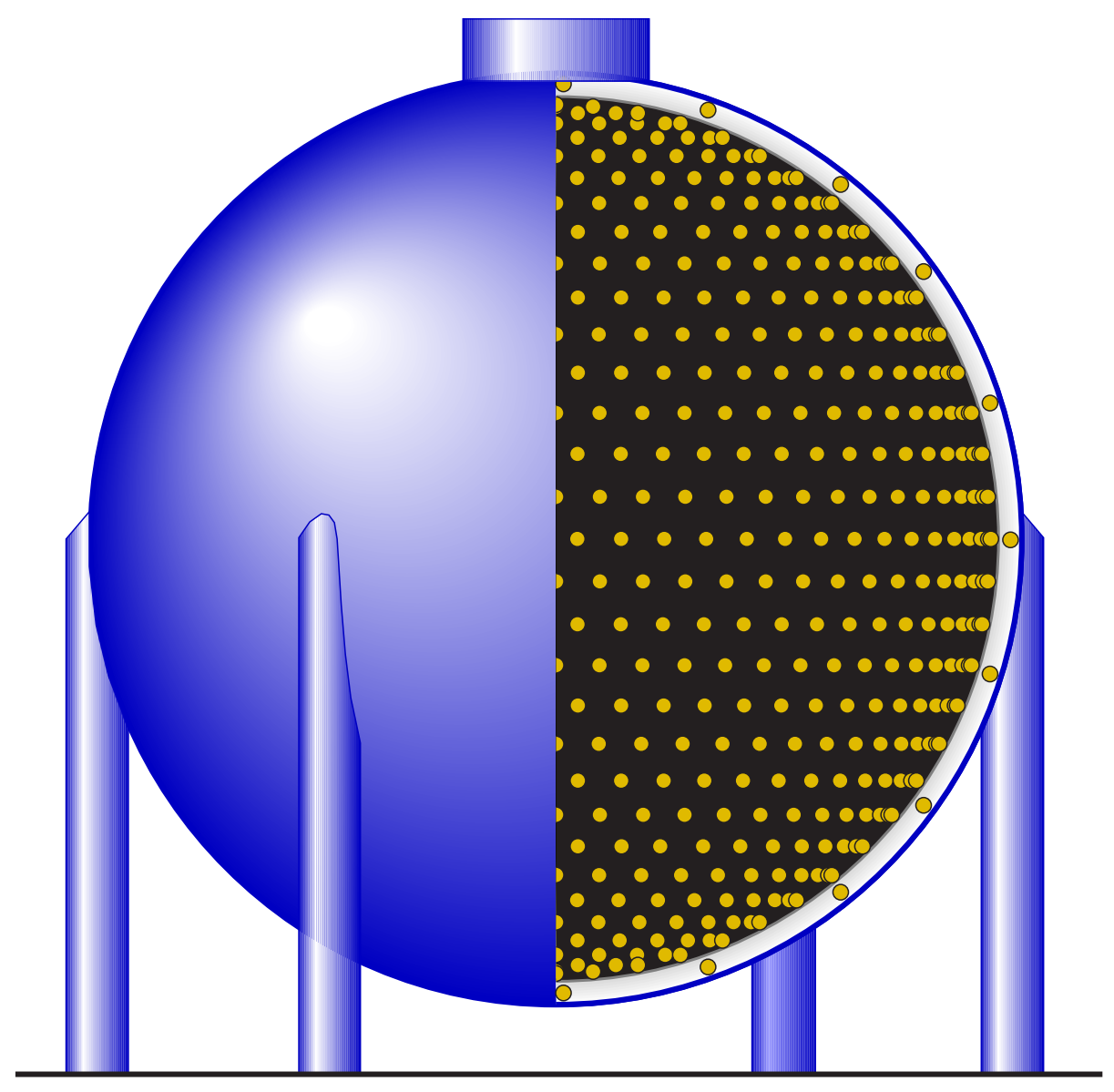

Figure 1.3: A schematic drawing of the MiniBooNE detector. 
which is two-thirds of a calender year. The sensitivities discussed above assume the experiment receives $5 \times 10^{20}$ protons per year. This Booster experiment is compatible with the Fermilab collider and MI programs. The Booster must run at $7.5 \mathrm{~Hz}$ to accommodate the MiniBooNE and collider programs simultaneously. The current schedule calls for data-taking to begin by the end of calendar year 2001 . 


\section{Chapter 2}

\section{Overview of Detector Design}

\subsection{Site Description}

The MiniBooNE site location is shown in Fig. 2.1. Protons from the Booster are extracted near MI-10 and transported to the Target Hall, which will contain a target within a focusing system, followed by a $\sim 50 \mathrm{~m}$ long pion decay volume. The resulting neutrino beam will point to the MiniBooNE detector, which is located $\sim 550 \mathrm{~m}$ north of the pion production target and southwest of the Leon Lederman Science Center. A possible future detector could be located north of Pine Street.

The local site plan is shown in Fig. 2.2. The MiniBooNE detector will be located near the NuMI beam line and will share the NuMI access road. The MiniBooNE detector facility, as shown in Fig. 2.3, will consist of a 40-feet diameter tank that stands in a 45 -foot diameter cylindrical vault with room above the tank for housing electronics and utilities.

\subsection{Detector Elements}

The MiniBooNE Detector Plant consists of two main elements: the Detector Containment and the Support Plant. The Detector Containment includes a spherical tank with access portal that sits in a cylindrical vault. The Support Plant lies above the Detector Containment and includes area for tank access, utilities, and electronics. 


\section{BooNE Detectors and Site Layout}

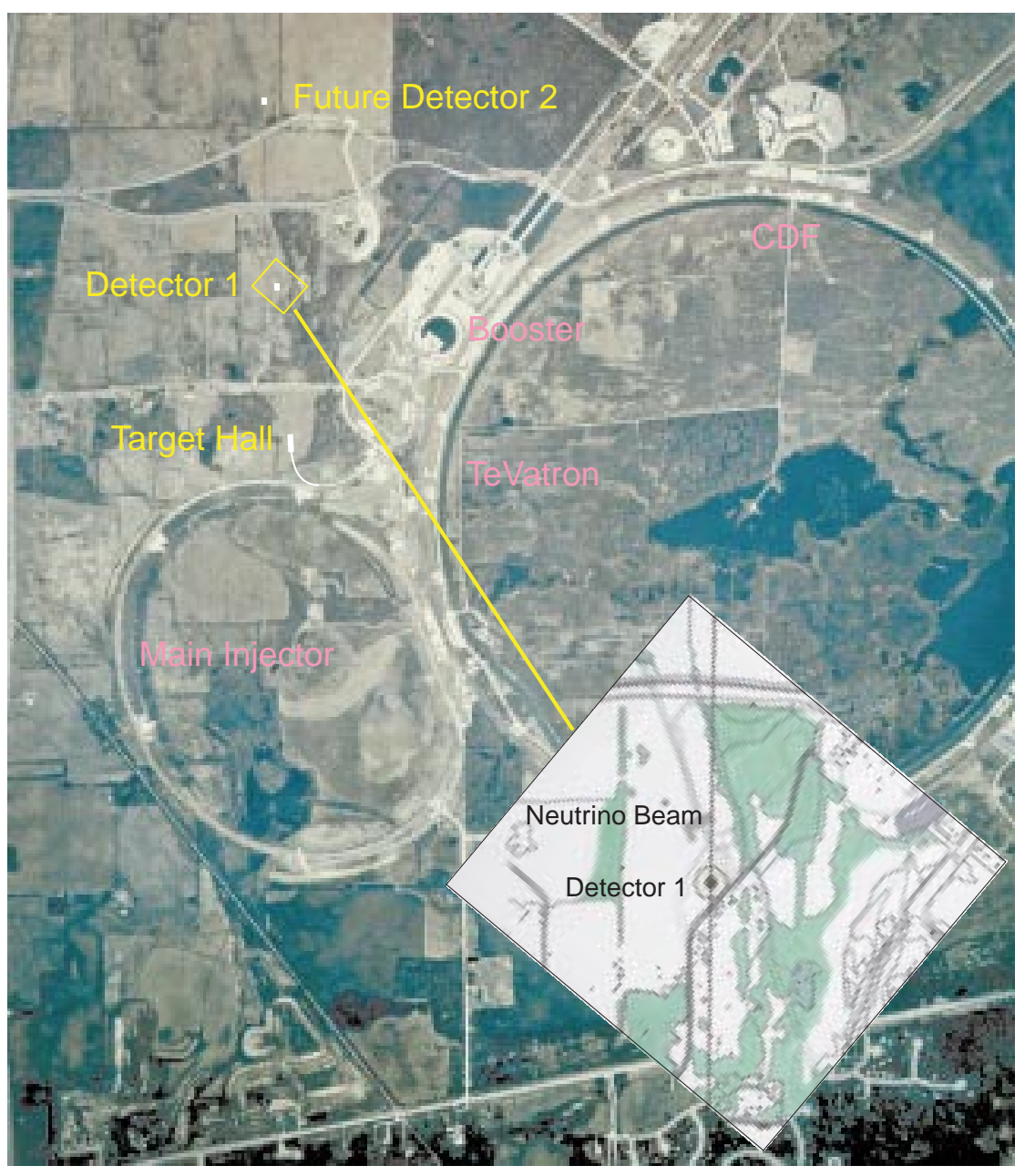

Figure 2.1: The MiniBooNE site location. 


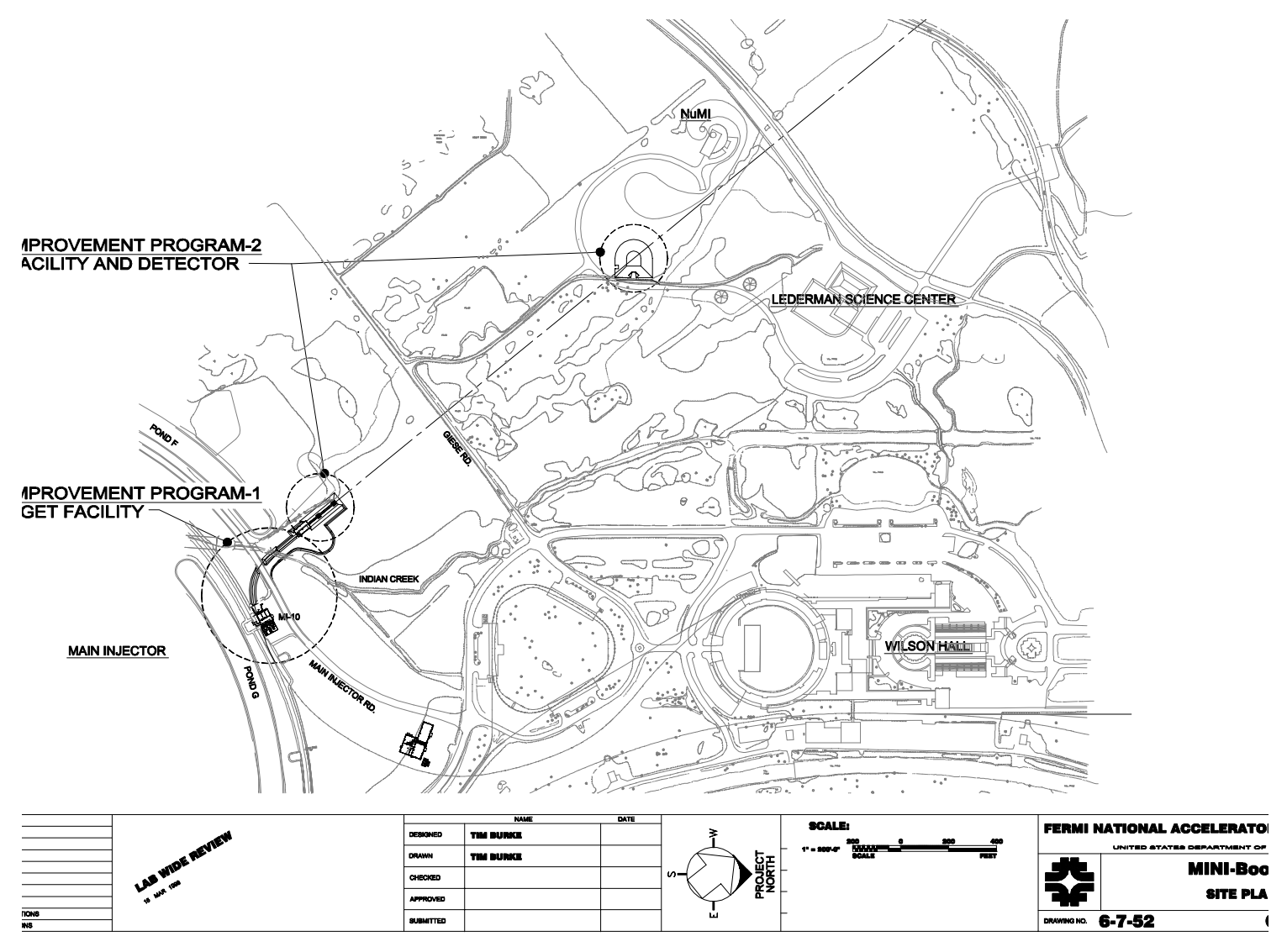

Figure 2.2: The MiniBooNE local site plan, showing the location of the $8 \mathrm{GeV}$ Fixed Target Facility and the MiniBooNE Target Facility and Detector. 


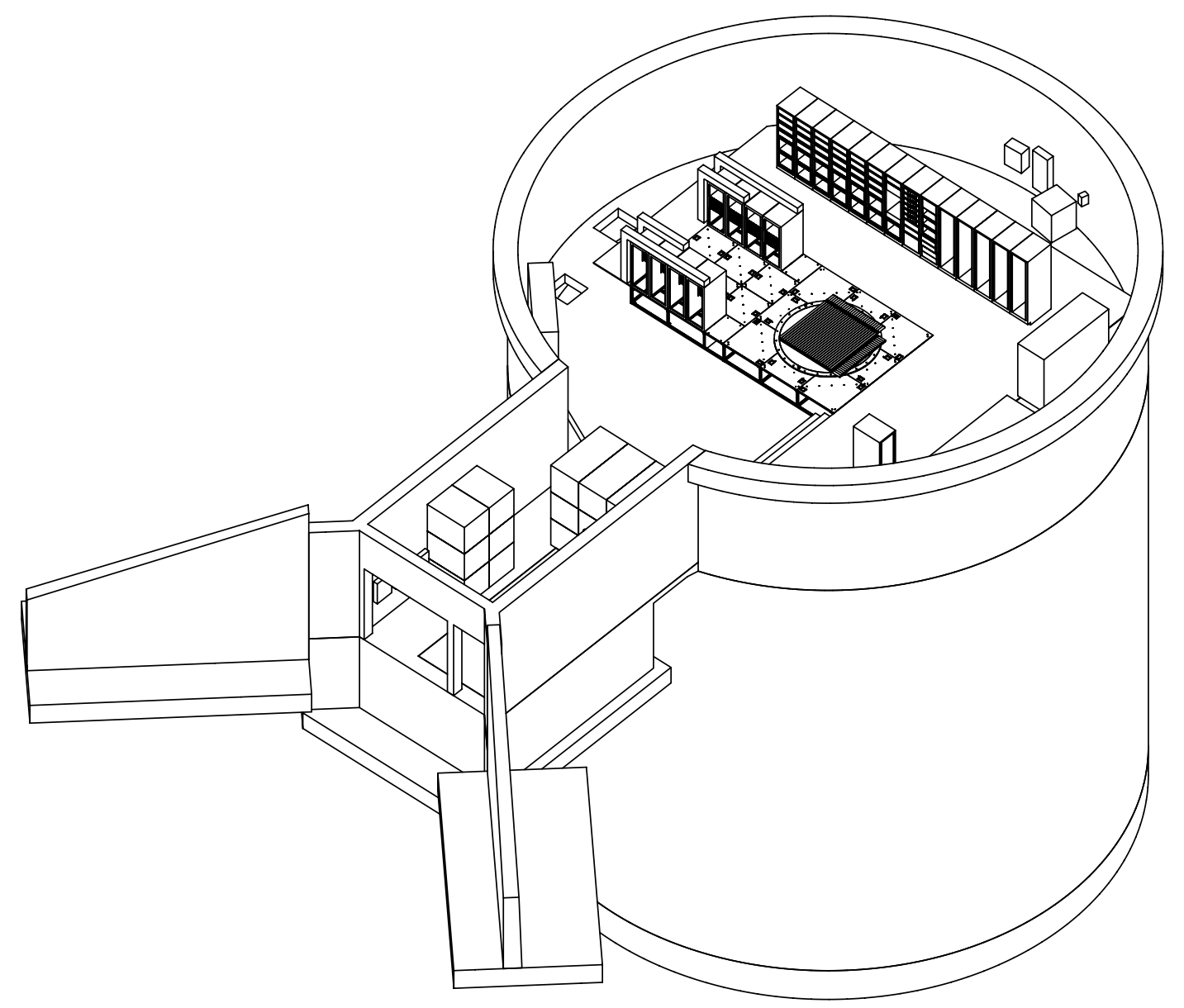

Figure 2.3: The MiniBooNE detector facility, consisting of the Detector Containment and Support Plant. 


\subsubsection{Cylindrical Vault}

The cylindrical vault will house the detector tank and will serve as the secondary containment for the oil. The vault will also provide the means for personnel access during the installation of phototubes. The excess soil from the excavation of the vault will be stored and reused as overburden for the detector enclosures to provide shielding from cosmic rays.

\subsubsection{Spherical Tank}

The spherical tank will have an inside diameter of 40 feet and will be constructed out of steel panels inside the cylindrical vault. Bosses that will be used to support the phototube support structure and cable plant will be welded on the inside walls of the tank. An 8-foot diameter access portal is attached to the top of the tank and provides the principle means for equipment access to the inside. The diameter of the access portal is sufficient for all expected penetrations of the tank, including plumbing, cabling, and calibration lines. There will also be a personnel access port near the bottom of the tank. After the tank is filled with mineral oil, the oil level in the access portal will be stabilized below the penetrations by an overflow pipe that runs to the overflow tank. A small inflow of oil, when required, will make the oil level stable at the level of the overflow runoff.

\subsubsection{Spherical Tank Internals}

The phototube support structure will consist of an inner shell consisting of 640 aluminum plates on each which will be mounted two phototubes. The plates themselves will be mounted on a series of latitudinal rings supported from the bosses on the tank wall. The volume inside the aluminum plates will form the detector volume, while the outside volume will form the veto shield region. Therefore, the inside surface of the aluminum plates will be painted

black, while the outside surface will be painted white. There will be a total of 1280 8-inch detector phototubes, providing $10 \%$ coverage of the surface area, and an additional 2408 -inch veto phototubes mounted directly on the tank wall. Each phototube will have one RG58 cable carrying both the high voltage to the phototube and the signal from the phototube. The cables will penetrate the side of the access portal through close fitting holes in the penetration panel, which will be above the oil level.

Light from a laser located in the utility enclosure will be piped along fiber optic cable through an access portal penetration to four $10 \mathrm{~cm}$ diameter glass bulbs that are located at various positions in the detector volume of the tank. The bulbs will be filled with LUDOX and will disperse the laser light isotropically, so that the phototubes can be timed accurately relative to each other. In addition, there will be six small scintillator cubes (each $5 \mathrm{~cm}$ on a side) that 
will be used for tracking cosmic-ray muons.

The tank will be filled with oil through a 3-inch diameter pipe that connects to the bottom of the tank. An overflow pipe in the acess portal connects to a storage tank and defines the oil level. Nitrogen gas will be bubbled into the tank at various levels and will help maintain a small nitrogen overpressure in the access portal. The nitrogen will purge oxygen and water from the oil.

\subsubsection{Detector Enclosure}

The Support Plant is located above the detector tank at existing grade and consists of areas for electronics, utilities, and tank access. The utilities area includes a reservoir tank to accommodate the variations in oil volume due to small variations in temperature. It also includes the active plumbing elements necessary for oil and nitrogen circulation and the laser used for phototube calibration. The electronics area includes fast electronics, a data acquisition system, a farm of workstations, and a taping system, while the tank access area contains the phototube preamplifier electronics and will provide for personnel and equipment access to the detector tank. An HVAC system, located in and adjacent to the utilities area, will be provided for the enclosure and detector vault. The main entrance and exit will be near the reservoir tank. The electrical power required for operation of the MiniBooNE experiment is estimated to be $\sim 100 \mathrm{KW}$. It is necessary to separate the electronics power source from the power used in the utility building for pumps, plumbing, and laser calibration. No water cooling is required.

\subsection{Electronics and Data Acquisition}

The phototubes, preamplifier boards, and digitization electronics from LSND will be reused in MiniBooNE. This system has a clock rate of $10 \mathrm{MHz}$, sufficient memory available for $200 \mu$ s of data before being overwritten, and is described in detail elsewhere [6]. Whereas LSND uses an updating sum of hit phototubes to generate a trigger, with MiniBooNE this is no longer necessary because of the short $\left(10^{-5}\right)$ duty factor. A precursor signal from the accelerator will be used to record data for a period of about $20 \mu$ s around each $1.6 \mu$ s beam spill. In addition, there will be other triggers used for calibration.

The MiniBooNE data acquisition system will be based on the LSND system. The 14 crates of electronics will be read out by monoboards located in each crate, and the data will be sent to the multi-processor computer that will build and reconstruct the events in real time. The reconstructed events will then be written to disk and logged to tape. A high-speed link will connect the control room located in Wilson Hall to the multi-processor computer, so that the experiment can be monitored and analyzed from the MiniBooNE control room. 


\subsection{Calibration}

The calibration system is designed to (1) provide information on the PMT response that is needed as input for the event reconstruction and particle identification calculations, (2) calibrate the position, energy and direction determination of the reconstruction program using stopping cosmic ray muons and Michel electrons from muon decay and (3) measure the attenuation of light by the oil in the detector tank.

The cosmic ray muon calibration system consists of a muon tracker above the detector and scintillator cubes inside the detector. The muon tracker will give the direction of cosmic-ray muons while the cubes will give a precise position measurement of stopping muons and decay electrons.

The laser calibration system will provide short pulses of light from a tunable dye laser to 4 flasks at various locations in the detector. The system is very similar to the system used successfully in LSND. It is used to determine phototube time-offsets and gains and to determine time-slewing corrections.

The oil monitoring system will measure attenuation of light as a function of wavelength in the detector. 


\section{Chapter 3}

\section{BooNE Detector Plant}

The MiniBooNE Detector Plant is the complex of structures needed for the detection and counting of neutrinos approximately $550 \mathrm{~m}$ from the neutrino source. The purpose of the detector plant is to hold the target mineral oil and photomultiplier tubes (PMTs) as well as house the necessary data acquisition electronics and oil pumping apparatus. It must also facilitate the initial assembly procedures and provide for secondary containment of the mineral oil. An overview of the plant is shown in Fig. 3.1.

The MiniBooNE Detector Plant (BDP) consists of two subplants: the Detector Containment Plant (DCP) and the Support Plant (SP). The DCP consists of the detector tank and related items (tank vault, access portal, etc.) and the SP consists of the structures above the detector tank (the access area directly above the detector tank and the areas for oil plumbing and electronics.)

The list below shows the names and hierarchy of the BDP parts.

- The Detector Containment Plant (DCP) consists of these items:

- detector tank,

- access portal, and

- tank vault.

- The Support Plant (SP) consists of:

- tank access area,

- utility area, and

- electronics area. 


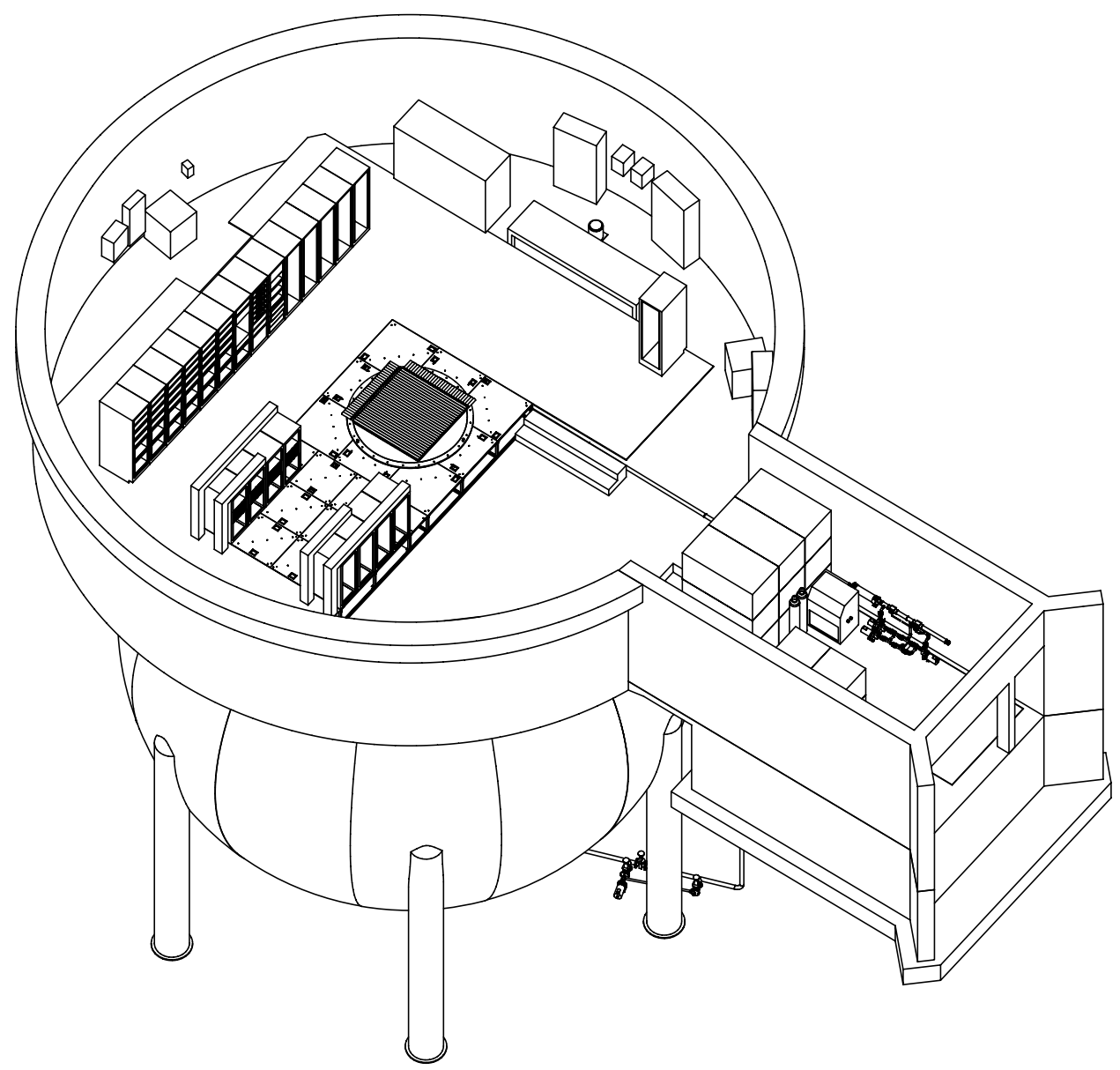

Figure 3.1: The MiniBooNE Detector Plant. Covering structures and earth overburden are not shown. 


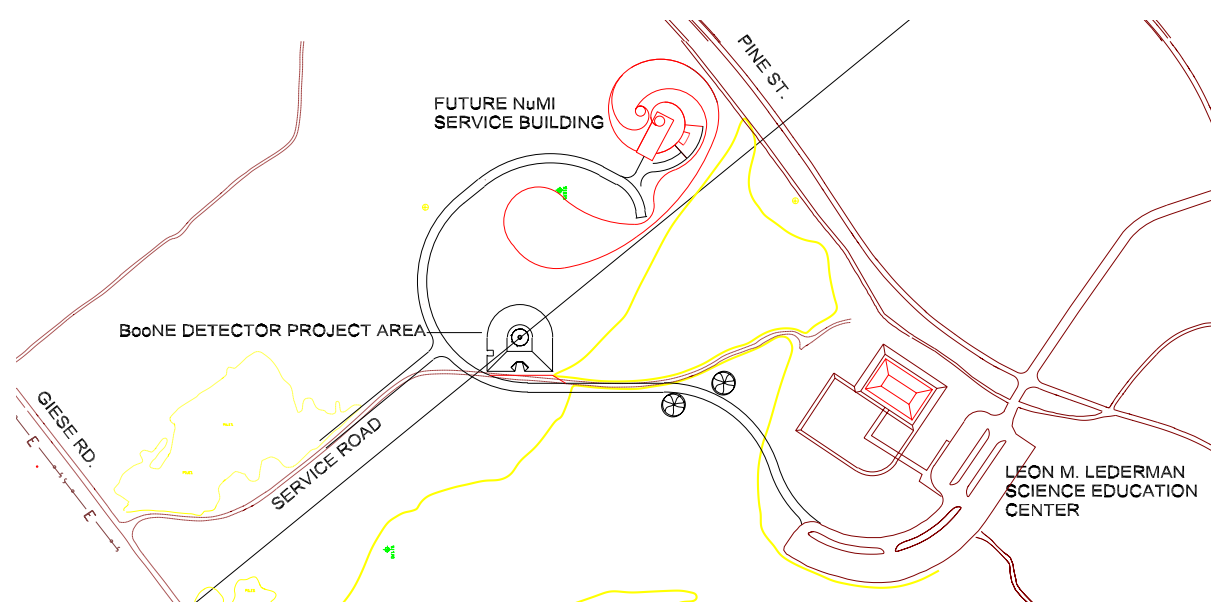

Figure 3.2: The site plan for the MiniBooNE Detector Plant. The service road is shown as planned for the final configuration. FNAL project north is to the right.

\subsection{MiniBooNE Detector Plant Site}

\subsubsection{Location}

The MiniBooNE Detector Plant is located approximately $550 \mathrm{~m}$ north of the neutrino production target along the nominal neutrino flight path as is shown in Fig. 3.2. This site is approximately $260 \mathrm{~m}$ southwest of the existing Lederman Science Education Center. It is a relatively flat and clear area and does not encroach on wetland areas.

\subsubsection{Soil Analysis}

The soil in the area (known fairly well due to recent surveys) consists of silty clay glacial till down to approximately 60 feet where a bedrock layer begins. The excavation for this project does not penetrate this bedrock. The water table varies from 5-15 feet below the surface depending on the local rainfall history and was considered in the construction plan.

\subsubsection{Access}

Access to the BDP area during the construction phase is via an existing service road as shown in Fig. 3.2. This road was reworked in order to provide access to the planned NuMI service building, also shown in Fig. 3.2. This work is not expected to interfere with the MiniBooNE construction schedule. The finished road configuration will allow access to areas immediately adjacent to the BDP 
for parking and material unloading. Construction vehicles access the area from the south end of this service road via Giese Road. After construction, access will be available to light vehicles from the extension of the road to the Lederman Science Center.

\subsubsection{Utilities/Communications}

The utilities/communication lines needed at the BDP site are:

- Electrical service with a capacity of $100 \mathrm{~kW}$.

- $100 \mathrm{Mbit} / \mathrm{s}$ ethernet.

- Phone Service.

- Accelerator Monitoring Signals.

- Fire protection.

The electrical service was tapped at the Lederman Science Center and routed to the site via a duct bank. This duct bank is used also to transport fiber ethernet links and phone service. A conduit is installed along a (fairly straight) line from the target hall to the detector. This conduit contains several cables required for fast monitoring and time synchronization with the neutrino target area.

\subsection{Detector Containment Plant}

The Detector Containment Plant consists of the spherical detector tank encased in a cylindrical tank vault as is shown in Fig. 3.3. The center of the tank is approximately 5 feet above the nominal center of the neutrino beam and is about 20 feet below the existing site grade.

\subsubsection{Tank Vault}

The tank vault provides structural support for the load above the tank and serves as a mineral oil secondary containment area.

The vault is cylindrical in shape with height of 43 feet and inner diameter of 45 feet. The walls of the vault are 1.5 feet thick concrete supported by soldier piles with lagging. Cast-in-place horizontal compression rings are installed in the wall to efficiently resolve the loading. A concrete floor for the vault was castin-place to support the filled tank. The top of the vault is spanned by precast and cast-in-place elements and serves as the floor of the access area above the tank vault.

The vault is surrounded by an impermeable membrane for groundwater control and mineral oil secondary containment. A groundwater sump is installed to remove collected ground water. 


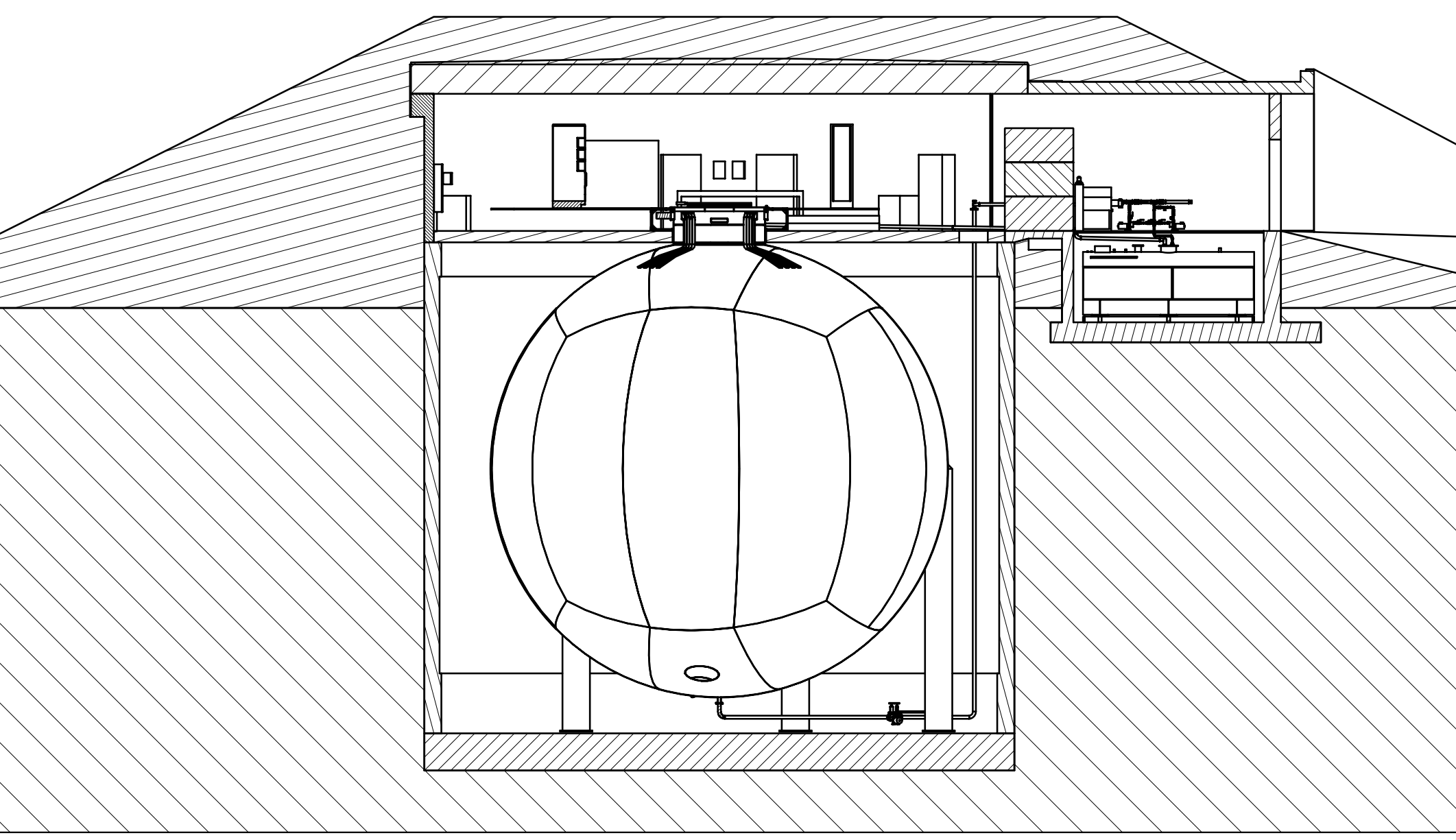

Figure 3.3: The Detector Containment Plant. 
Access to the vault are is via a spiral stairway mounted on the vault wall. This access is used for personnel during the installation phase and, later, for inspection of the tank and service of the sump pumps. These are described in Sec. 3.3.1.

\subsubsection{Oil Secondary Containment and Monitoring}

This vault system with the impermeable liner will be completely adequate for a mineral oil secondary containment system. In the unlikely event of a breech in the detector tank or adjacent oil plumbing, any spilled oil will pool below the tank and will be completely contained within the tank vault. A monitoring system and secondary containment pump is installed in the vault area below the tank. A sampling of this area will be performed regularly.

\subsubsection{Tank}

The detector tank (shown in Figs. 3.3 and 3.4) is a spherical vessel of 40 feet inside diameter (which gives a volume of approximately 250,000 gallons) with a portal (described in next section) to allow access from the top. The design of the tank and access portal is based on the applicable rules contained in the latest issues of API 620 and NFPA 30 and all new materials are used. The thickness of the tank walls is dictated solely by structural considerations. The inside of the tank is covered by a non-reactive coating. The outside of the tank will be painted, but since humidity-controlled air will be the only substance in contact with the outer surface of the tank, only minimal protection will be needed. No cathodic protection will be required.

The tank is supported by steel legs fixed to the concrete floor of the vault. The top of the spherical portion is approximately level with existing site grade.

A 3in fill pipe penetrates the tank at the lowest point. This pipe is used to pump the oil into the tank during the initial fill and during steady state running when the oil is recirculated. It will also be used for an emergency or decommissioning pump out. It terminates inside the tank into a large-area velocity-reduction flange. A 3 feet diameter personnel access port is installed on the bottom of the tank slightly offset from center. This port will be used only during the construction and installation phase.

All other penetrations will be through the access portal described in the next section.

\subsubsection{Access Portal}

The access portal is a cylindrical-shaped extension attached to the top of the spherical portion of containment tank as shown in Figs. 3.3 - 3.6. The purpose of this portal is to allow access for materials and equipment into the tank dur- 


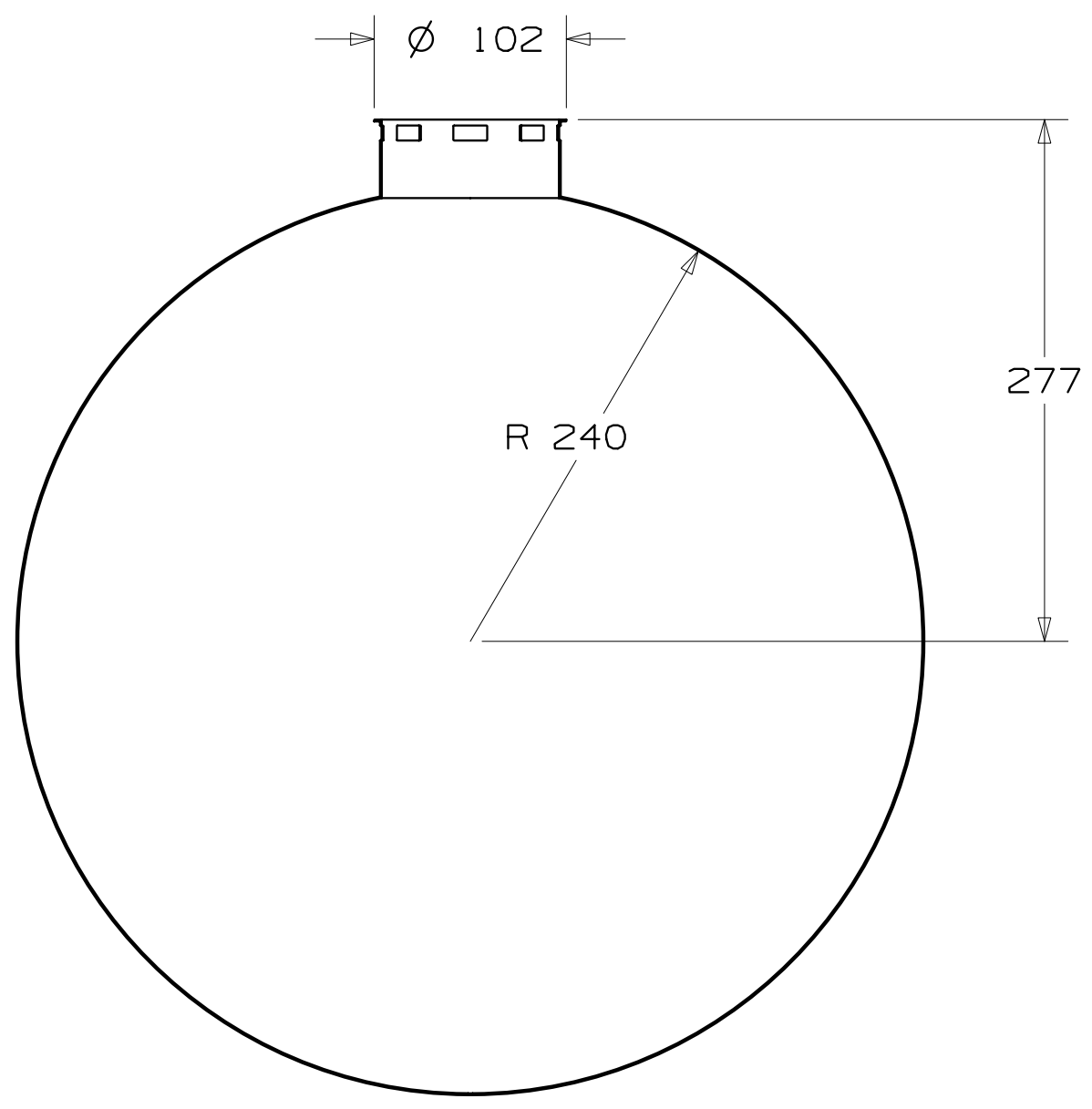

Figure 3.4: The detector tank in section. The support legs are not shown in this view. Dimensions are in inches. 


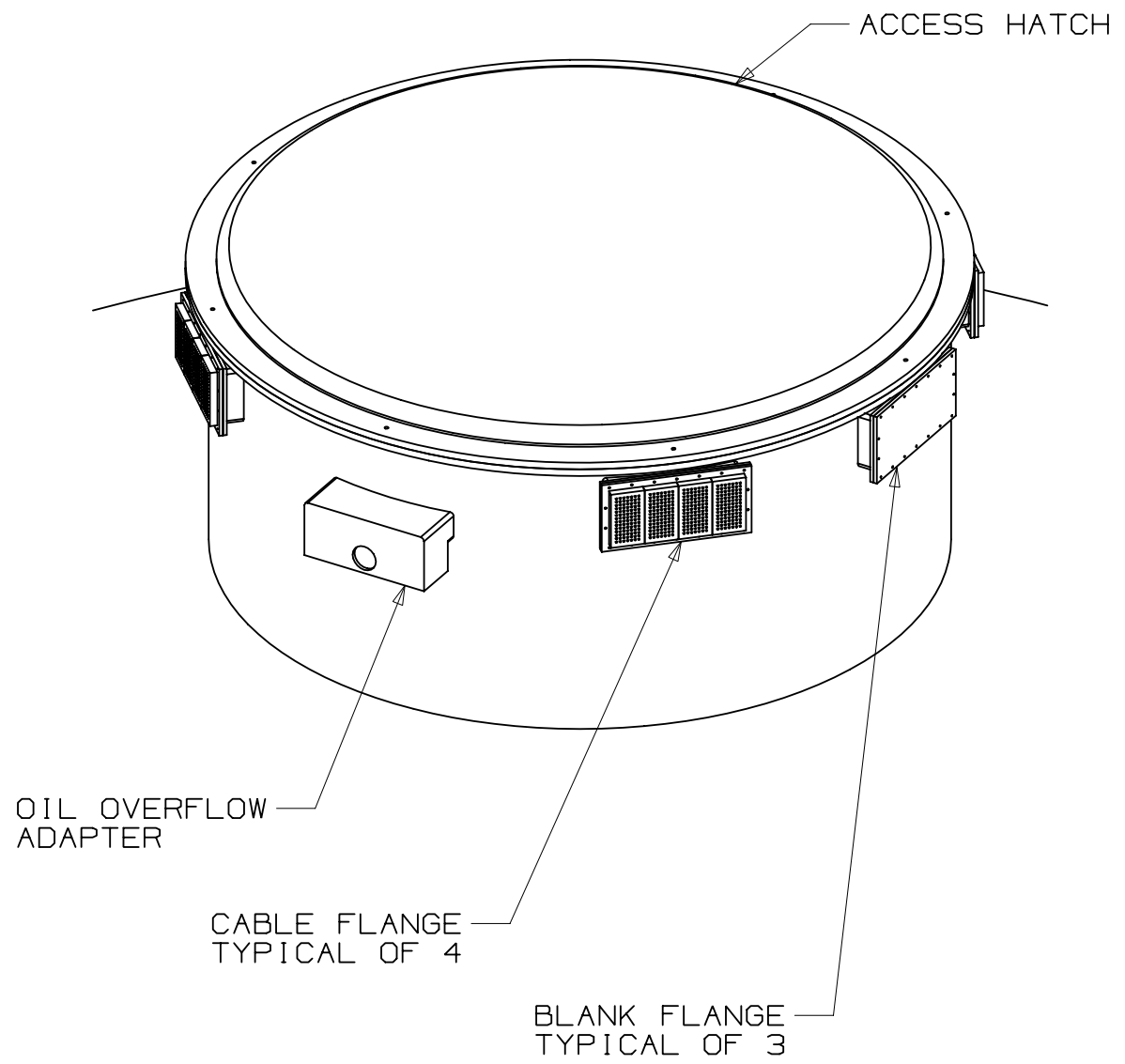

Figure 3.5: A perspective view of the detector tank access portal.

ing installation of internals and to provide a convenient surface for cable and plumbing penetrations.

The access portal is 8 feet in diameter and 3.4 feet in height. It extends through the roof of the tank vault into the access area situated immediately above the tank. The top of the access portal is covered by the access hatch. The hatch covers the entire top circular cross section and, when opened, allows access to the tank through the entire 8 feet diameter of the access portal. When closed and sealed, it will maintain a slight overpressure of nitrogen. It uses a simple flange-bolts design and is constructed of aluminum. There will be no penetrations through the access hatch. All plumbing and cable penetrations are made through the wall of the access portal.

There are eight ports spaced at equal intervals around the side of the access 

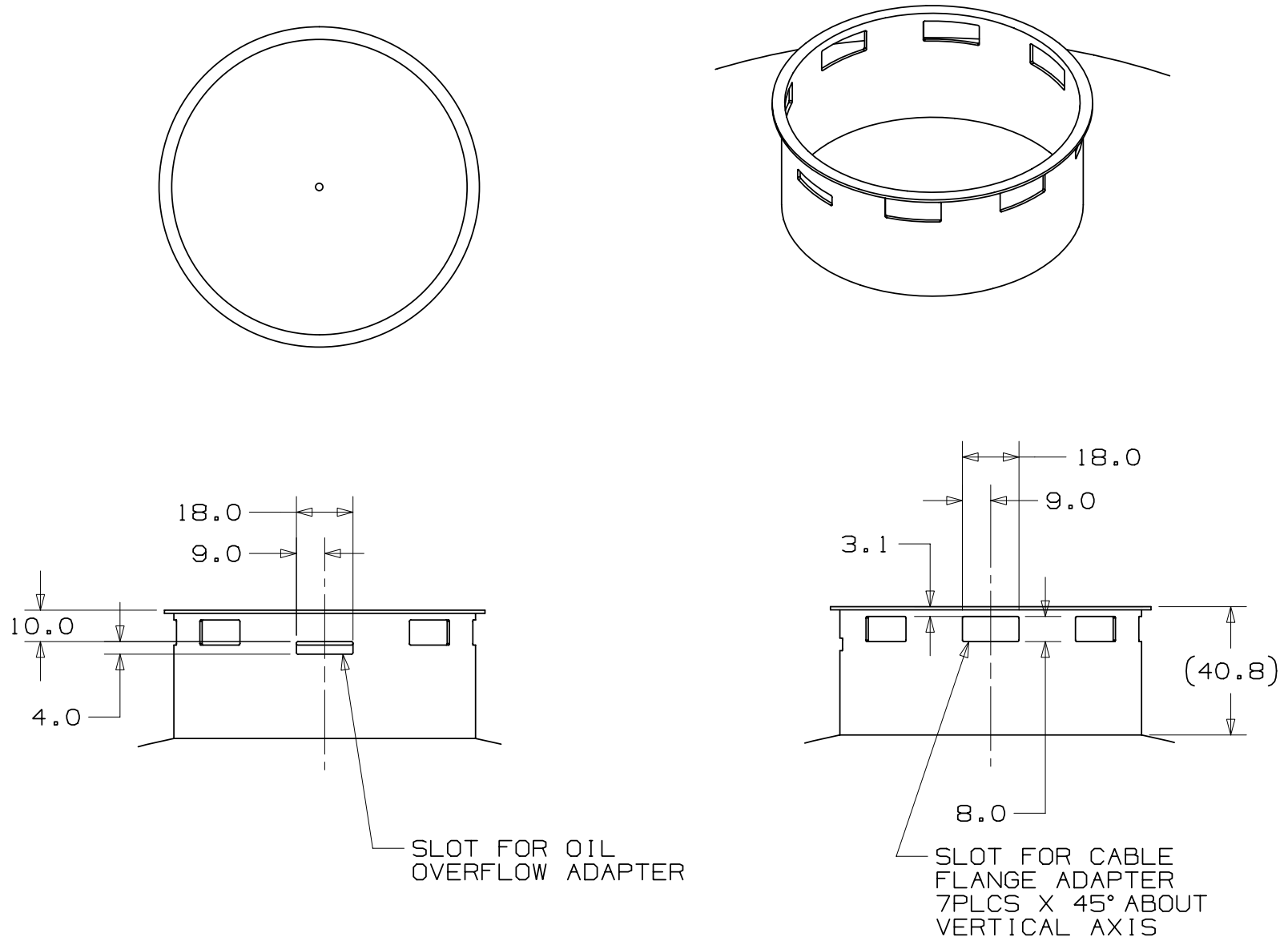

Figure 3.6: Details of the detector tank access portal. Dimensions are in inches. 
portal (see Figs. 3.5 and 3.6). Seven are 8in tall by 18in wide and located 3in down from the top of the portal. Four of these ports allow access for the PMT signal cables and three will be used for oil and nitrogen plumbing and calibration cables. A custom template is fabricated for the specific cable or pipe group that it accepts. When complete and sealed, these ports will also maintain a slight nitrogen overpressure.

There is one additional penetration for oil outflow. The oil outflow will be through a port 4in tall by 18in wide that is installed on the access portal wall just below the other penetrations. This port will connect to a welded box that connects to a 3in flanged pipe. The oil outflow plumbing connects to this pipe. This outflow port sets the level of the mineral oil. It is the only penetration through the access portal below the level of the mineral oil inside the tank.

\subsection{Support Plant}

The Support Plant (SP) consists of the structures above the tank that contain the electronics, oil plumbing, and calibration equipment. These structures also provide for access into the tank and for staging areas during assembly and installation. They are designed to allow convenient routing of cables, plumbing, and conditioned air under a raised computer floor system that is installed throughout most of the SP.

The support plant is contained in the cylindrical area above the tank vault and the entrance corridor. The electronics and access areas are in the cylindrical area and the utility area is in the corridor. The utility and electronics area are kept separated as much as is possible to reduce noise induced in the electronics from the oil pumps and cooler. A plan view of the SP with the main areas labeled is shown in Fig. 3.7.

\subsubsection{Tank Access and Electronics Area}

The tank access and electronics areas are situated directly above the detector tank vault. The access area provides an open space for access into the access portal and tank vault. This area contains the preamplifier electronics for the detector PMTs. A raised-floor "faraday-cage" structure surrounds the access portal and provides a shielded area for the PMT signal cables. This faradaycage is approximately 2 feet high and sits flush with the computer floor installed throughout the electronics area. Eight racks containing the preamplifier electronics sits atop the faraday-cage. These electronics accept cables from the PMTs.

The electronics area is also located in the cylindrical structure above the tank. It contains, as shown in Fig. 3.8, 14 19-inch racks (7-feet tall) that will house these items:

- QT DAQ boards (in VME crates), 


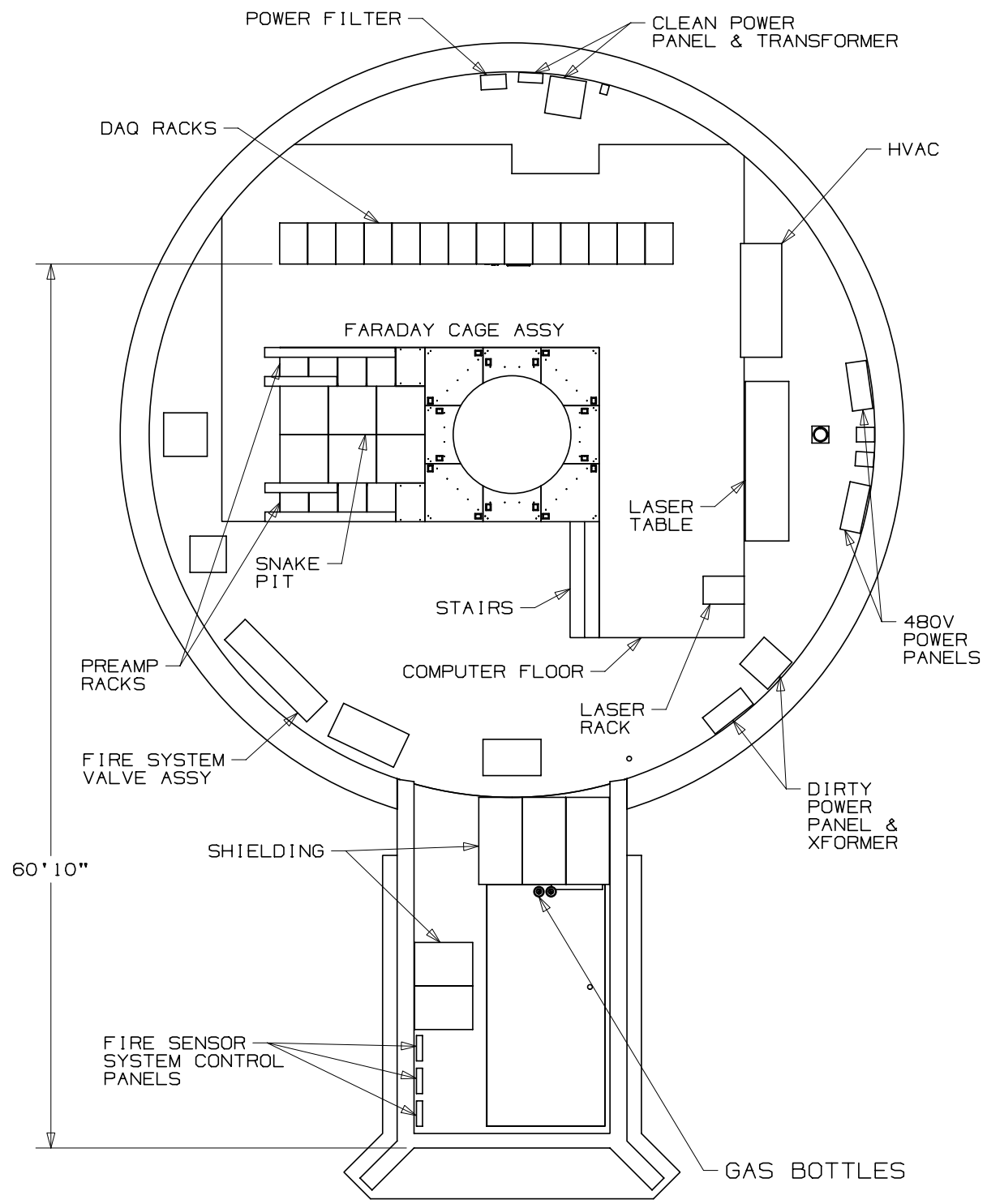

Figure 3.7: The MiniBooNE Support Plant in plan view showing the access, electronics, and utility areas. 
- data acquisition computer and tape unit,

- PMT and preamplifier power supplies,

- accelerator and target hall monitoring equipment,

- electronics and HV for the calibration system.

A raised computer floor is installed in this area. The area beneath the floor will be used to route cables from the preamplifier electronics to the QT boards and to route cool air to the electronics racks from below. "Clean" electrical power will be provided to this area.

\subsubsection{Utility Area}

The utility area is located to the side of the entrance corridor and is shown in Fig. 3.9. It contains the oil overflow tank, oil pumps, oil chiller/heat exchange unit, nitrogen supply. The utility area is isolated as much as is practical from the electronics area and uses an isolated electrical circuit.

The oil overflow tank is an existing (at LANL) stainless steel tank with 2500 gal capacity and is shown in Fig. 3.10. It is set into a concrete pit in the entrance corridor with the top below the level of the mineral oil in the detector tank so that overflow oil from the detector tank will run to the overflow tank under the influence of gravity alone. The pit will serve as secondary containment for the overflow tank. The pumps to move the oil from the overflow tank as well as the oil chiller and heat exchange unit will be located in the utility area adjacent to the overflow tank. More details on the oil plumbing may be found in Sec. 3.5.3.

There will also be a supply system for the nitrogen that will be bubbled through the detector and overflow mineral oil volumes to purge oxygen.

\subsection{Overburden}

An earth overburden covers the MiniBooNE Detector Plant, as shown in Fig. 3.11, to a height of 24 feet above the existing surrounding grade. The earth is formed into a roughly truncated cone shape and centered over the detector tank. In this scheme, for all areas except around the entrance corridor, the minimum earth equivalent that a cosmic ray particle must traverse to enter the tank is $3 \mathrm{~m}$. With this overburden scheme the cosmic muon rate through the detector will be less than $10 \mathrm{kHz}$.

The overburden is held back from the access door via retaining walls as shown in Fig. 3.11. These are the only retaining walls required. 


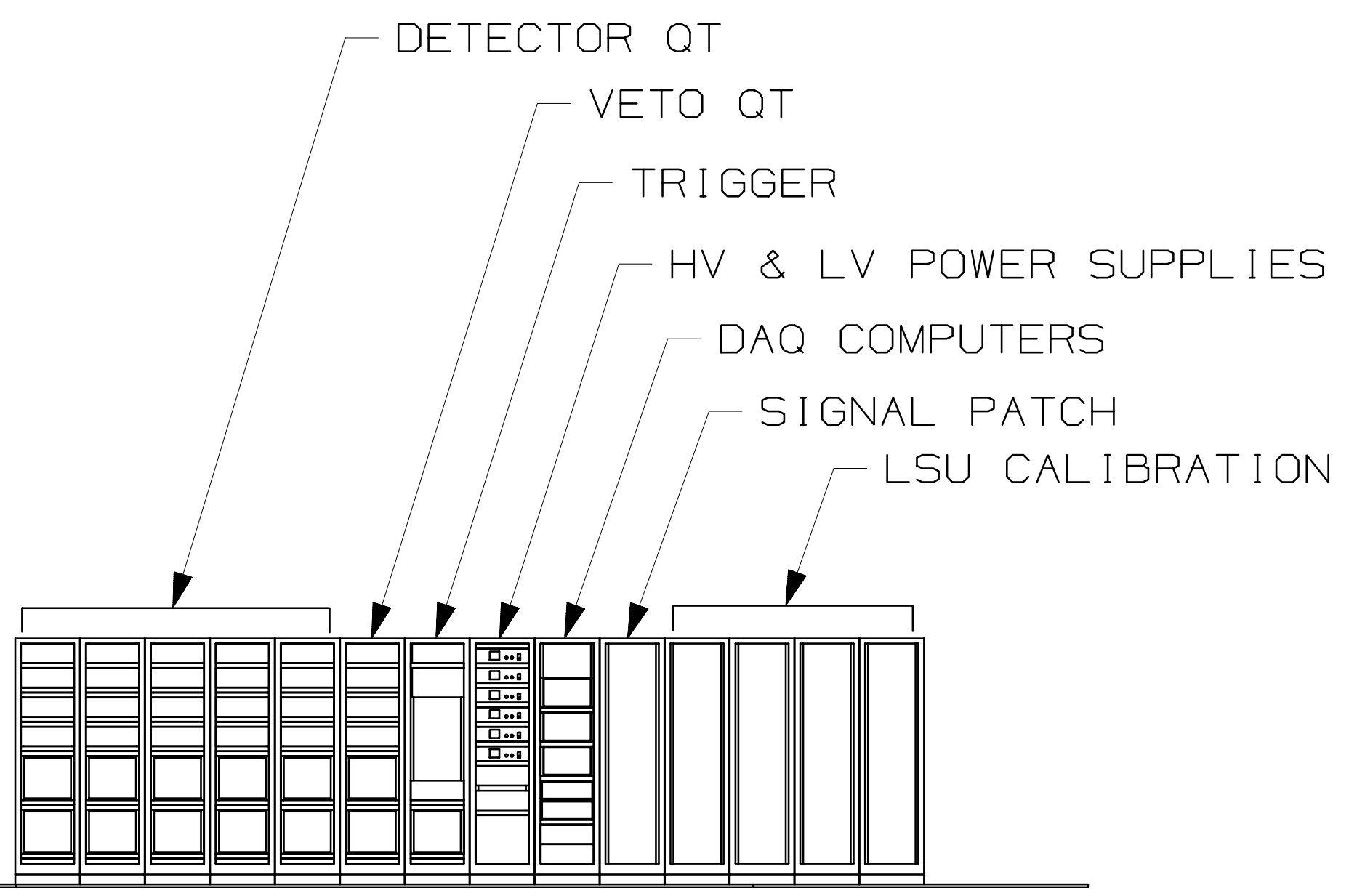

Figure 3.8: Elevation views of the Electronics Area showing the locations of the QT electronics racks, the power supply rack, the DAQ computer rack, the accelerator monitoring rack, and the calibration racks. 


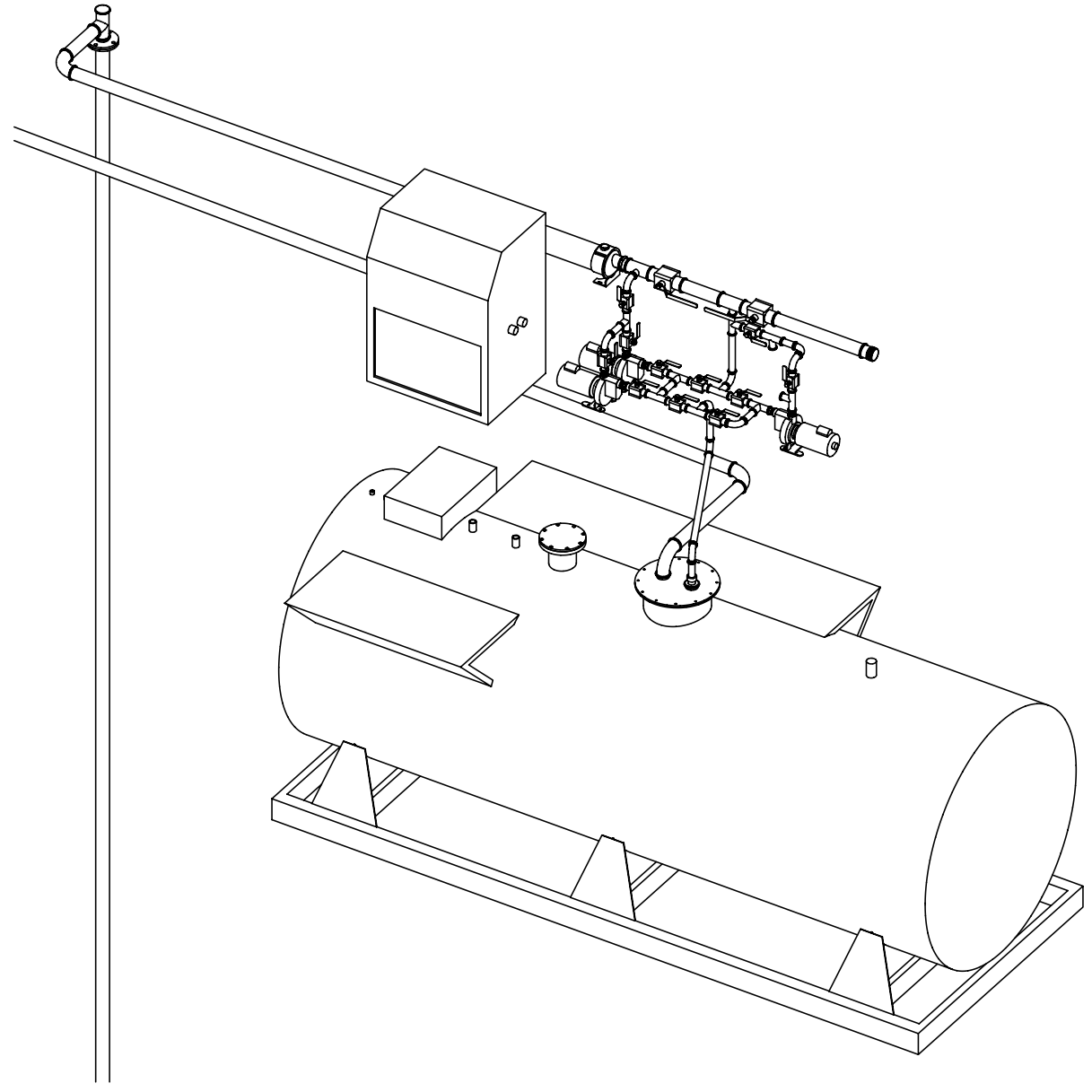

Figure 3.9: A view of the utility area showing the location of the oil overflow tank, oil pumps, oil chiller/heat exchange unit, and other utility components. 


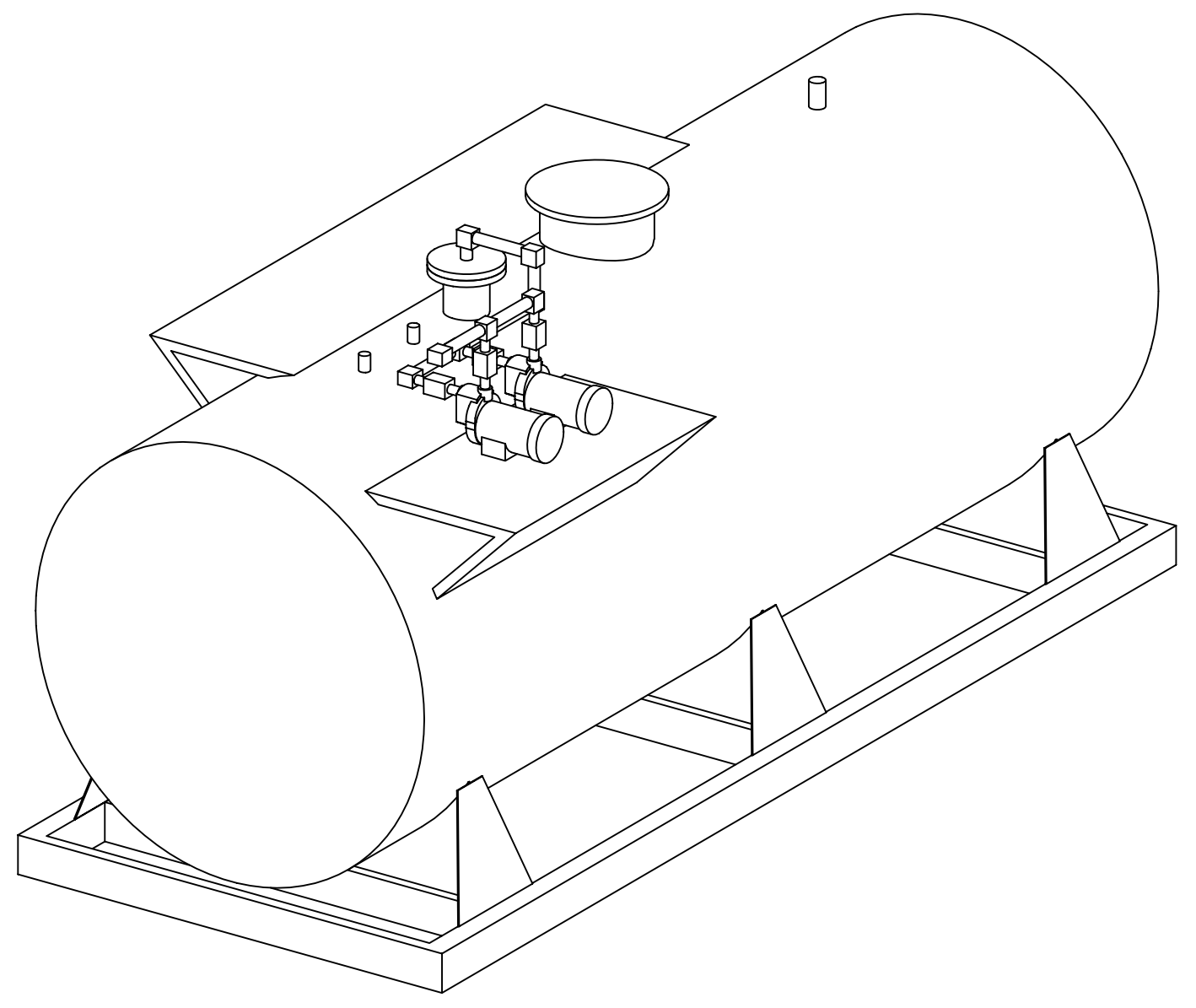

Figure 3.10: A perspective view of the oil overflow tank. 


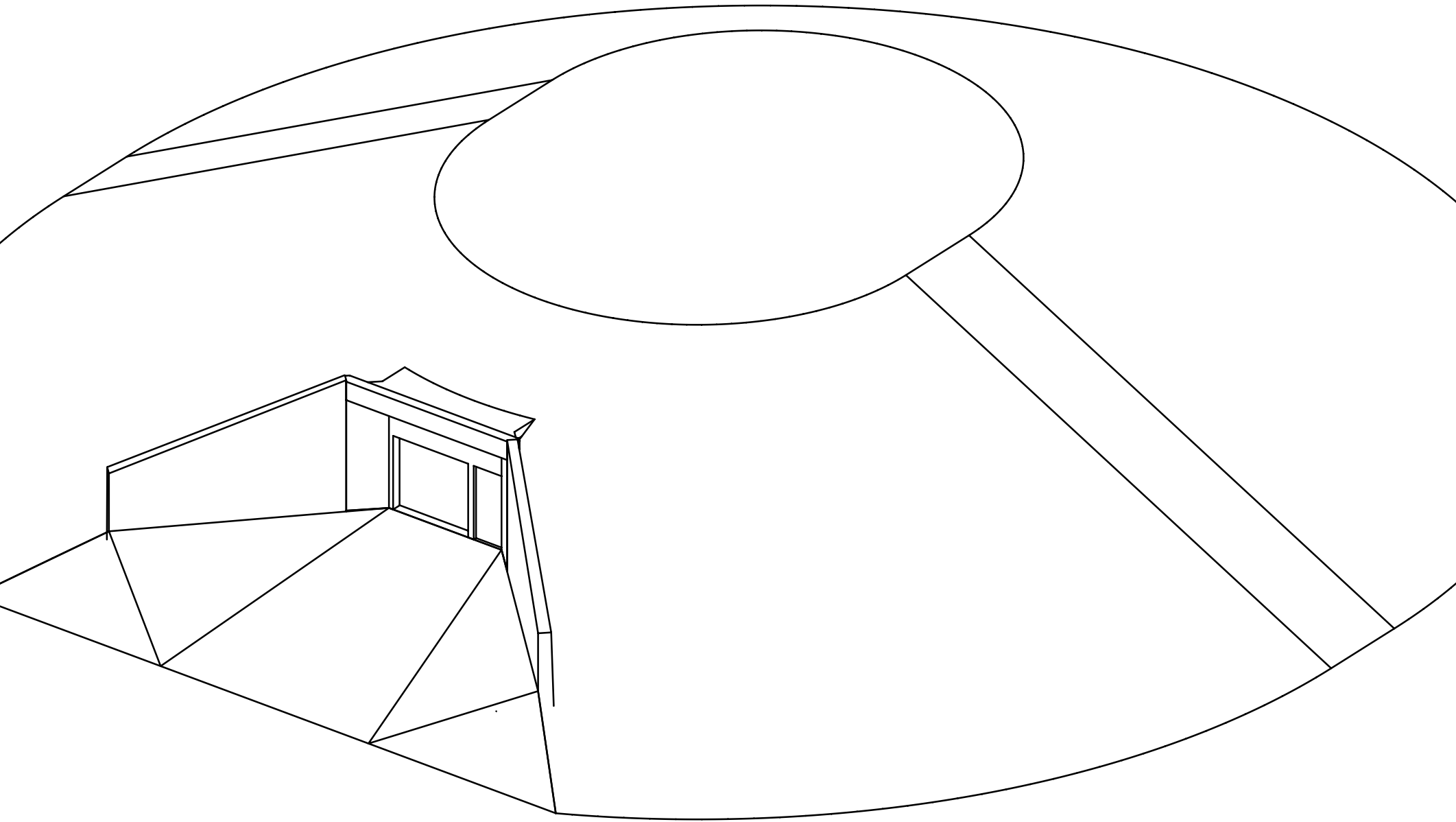

Figure 3.11: A schematic view of the MiniBooNE detector overburden. 


\subsection{Additional Details}

The following sections give more details on various aspects of the MiniBooNE Detector Plant.

\subsubsection{Heating/Cooling}

Temperature regulation of the Detector Plant will be accomplished via a large HVAC unit located adjacent to the Access Area in the cylindrical structure above the tank. Temperature and humidity controlled air is routed under the raised computer floor to provide cool air to the electronics. The air will return to the HVAC unit return vent through the areas above the raised floor with no additional ductwork.

\subsubsection{Electrical}

The MiniBooNE Detector plant requires electrical service of voltages $120 \mathrm{~V}$, $208 \mathrm{~V}$, and $480 \mathrm{~V}$ with total power capacity of $100 \mathrm{~kW}$. Details may be seen in Fig. 3.12. Power is supplied to the site in the form of a $13.8 \mathrm{kV}, 100 \mathrm{~kW}$ feeder, where it is split and routed to two service transformers. One transformer provides power for the "clean" power, used to for the electronics, and the other provides power for the "dirty power", used for the HVAC, oil pumps, lighting, and miscellaneous circuits. Special care is taken to provide proper and adequate grounding for sensitive electronic equipment.

\subsubsection{Oil Plumbing}

A schematic of the oil plumbing is shown in Fig. 3.13. The oil flows into the detector via a 3in line that attaches to the inflow penetration at the bottom of the detector tank that is described in Sec. 3.2.4. Flow studies indicated that, unless oil was supplied directly to the main volume of the tank, most of the circulation would be through the outer (veto) region. For this reason, the inlet line ends in the tank in a coaxial fitting that delivers most of the oil to the main volume. Another 3in line is attached to the outflow flange on the access portal (also described in Sec. 3.2.4). This 3in outflow line is routed to the oil overflow tank with a slope of $1 / 8$ inch per foot so that the oil pushed out from the detector tank will passively flow to the overflow tank. Oil from the overflow tank is pumped back to the detector via the 3in inflow line.

The oil inflow line passes through a heat exchange unit attached to a chiller with $10 \mathrm{~kW}$ capacity. This arrangement will allow for cooling the oil during the initial oil fill as well as during regular running.

After the tank is filled and the detector is turned on, approximately $600 \mathrm{~W}$ of heat (generated by the PMTs) will need to be removed. This heat will be dissipated into the cooled air of the tank vault and the tank will come to a 


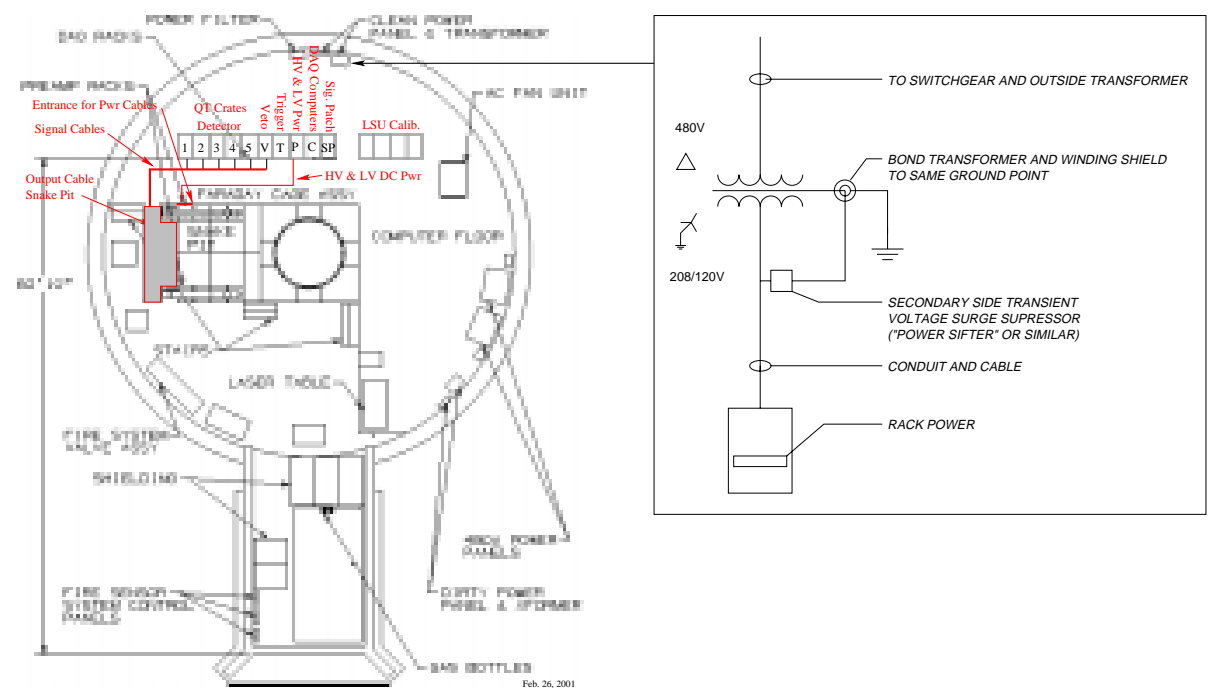

Figure 3.12: A schematic figure illustrating the electrical service requirements in the MiniBooNE detector plant.

steady state temperature of close to that of the surrounding earth (approximately $55^{\circ} \mathrm{F}$ ). However, if additional cooling is needed, the oil may be continuously recirculated at up to $10 \mathrm{gal} / \mathrm{min}$ through the chiller/heat exchange unit. Oil may be pumped from the overflow tank (which will be kept about 1/2 full) through the heat exchange unit into the detector tank through the oil inflow plumbing. This (cooled) oil will enter the main tank volume with fairly low velocity at the bottommost point of the tank. This constant inflow will push an equivalent amount of oil from the top into the oil outflow port which will then gravity flow to the oil overflow tank to complete the cycle. This process may continue around the clock, and the flow rate and/or temperature reduction of the oil will be adjusted to obtain the desired steady-state temperature. This will also ensure that the oil level remains defined by the overflow plumbing.

The coefficient of expansion for mineral oil is about $0.1 \%$ per $1{ }^{\circ} \mathrm{C}$. This overflow tank has capacity of 2500 gallons or about $1 \%$ that of detector tank capacity. The overflow tank will be kept about $1 / 2$ full so this implies that the allowed temperature range will be about $10^{\circ} \mathrm{C}$. This will be easily obtainable during steady-state running. During the initial oil fill and while temperatures come to equilibrium, the oil levels will be closely monitored and an empty tanker truck will be kept nearby the site to allow for adjustment.

An oil removal pump is installed in the inflow line at the bottom of the tank vault. This pump will be used to remove the oil for decommissioning. 


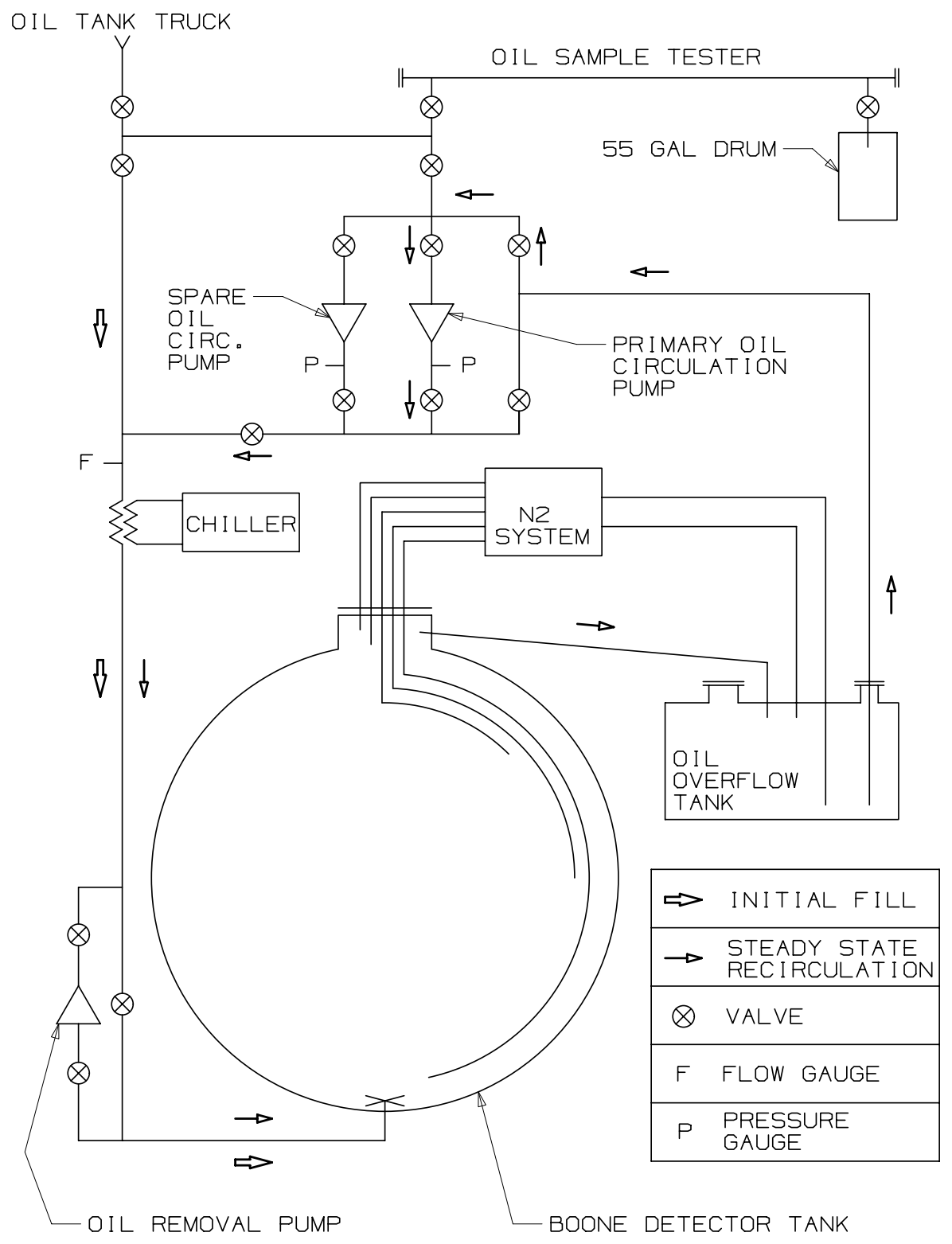

Figure 3.13: A schematic figure showing the oil plumbing scheme. 


\subsubsection{Signal Cables}

The signal cables originate inside the detector tank at the PMTs. The details of the cable routing inside the tank are given in Chapter 4 . The approximately 1520 signal cables exit the tank through four of the cable ports described in Sec. 3.2.4. There will be four subgroups of 98 cables within each of the cable ports. These 98 cables will be passed though a machined jig with one hole for each cable.

These 16 bundles of 98 cables each will be routed from the cable ports under the raised floor of the faraday-cage to the eight racks containing the preamplifier electronics. From these racks, another set of cables will be routed to the electronics area into the QT racks under the raised floor system.

\subsubsection{Internal Component Installation}

The internal detector components including necessary scaffolding are brought in through the access door and lowered into the detector tank through the access portal. Details on the installation of the tank internal components (PMTs, etc.) may be found in Chapter 4 .

\subsubsection{Oil Transportation and Fill}

After the internal detector components are installed, the mineral oil fill procedure will begin. The mineral oil will arrive at the MiniBooNE Detector Plant via tanker trucks. These trucks will unload near the access door. Each tankload of mineral oil will be tested thoroughly to ensure that any contamination in a particular tankload will not be allowed into the detector volume. The primary method to test for contamination will be to check the attenuation length of the oil.

There will be an apparatus located at the MiniBooNE detector site to make this attenuation measurement. The system will consist of a monochromatic light source tunable over a wide range of wavelengths. The oil sample will be in a long pipe which will be connected to the rest of the oil delivery plumbing as shown in Fig. 3.13. At the opposite end of the oil sample pipe from the light source will be a phototube to detect the intensity of the transmitted light. If the results from this attenuation measurement are satisfactory, the oil from this tankload will be pumped into the detector tank. 


\section{Chapter 4}

\section{Phototube Support Structure}

The photomultiplier tube support structure (PSS) includes the hardware for supporting the 1280 main and 240 veto photomultiplier tubes (PMT's), the optical barrier separating the main and veto oil volumes, the fixtures for support and strain-relief of the PMT cables, and support for the various in-tank calibration and monitoring systems. The dimensions of the sections of the PSS are chosen to uniformly distribute the main PMT's over the inner surface of the optical barrier and the veto PMT's over the tank wall.

\subsection{Design constraints}

The allocation of PMT's in the main tank and veto and the thickness of the veto region were determined by physics considerations and were arrived at using Monte Carlo simulation of signal and background events. The Monte Carlo studies used a full GEANT simulation, including tracking of individual Čerenkov and scintillation photons, with wavelength-dependent absorption, reflection, and detection efficiency. Analysis of events in the main tank indicated that at least $10 \%$ photocathode coverage (defined by treating the photocathodes as flat disks with diameter equal to the PMT diameter) was needed to provide the required particle identification quality. When tuned to the secondary requirement that veto and main channels not be mixed in the same electronics crate, a final number of 1280 main phototubes resulted.

The total number of PMT's available is limited by cost and the number of available electronics channels. This allows 240 veto PMT's, and the issue is whether the veto region then yields sufficient light to reject muons from cosmic rays and beam-neutrino interactions outside the tank with efficiency $>99 \%$, the design goal. Monte Carlo studies, including conservative estimates of the 
albedo of the painted surfaces in the veto (see Sec. 4.3), indicated that the light yield with a $35-\mathrm{cm}$ veto thickness was high enough to allow reasonable thresholds in the face of noise and light leaked from the main tank. Separate calculations showed that shadowing of muon tracks by the struts and cables that will penetrate the veto region were negligible.

It is not possible to distribute the phototubes over the sphere with exact uniformity. For reasons of structure and ease of installation, the PMT's are deployed in evenly-spaced horizontal rows. The number of tubes in each row must be even and is chosen to maintain horizontal spacing that is as close to uniform and as close to the vertical spacing as possible. The final variable in the layout is the horizontal "clocking" of each row. Monte Carlo studies showed that this is not a serious issue. In any case, adjacent rows typically do not match in number of tubes, so any initial correlation is lost as the rows go around the tank. Near the equator, however, there are several rows that could line up. For this reason, even-numbered rows start with a tube at $\phi=0$, and odd-numbered rows start with the first tube shifted by half the horizontal spacing of the tubes.

Most of the PMT's used in MiniBooNE are 8-inch Hamamatsu R1408's recycled from LSND. However, as discussed in Chapter 6, we have purchased 330 newer R5912 tubes (also 8-inch). As these new tubes have better time and charge resolution, all will be used in the main tank. They have been assigned random positions in the tank, with a distribution of new and old tubes shown in Fig. 4.1.

\subsection{Structure}

Besides the obvious requirement of adequate strength, the PSS must be straightforward to install and must be tolerant of deviations of the tank wall from a perfect sphere. (Industry standards limit such deviations to $1 \%$, or about 5 inches.)

The basic design of the support structure is shown in Fig. 4.2. The optical barrier and inner PMT's are mounted on a set of latitudinal hoops or "lats," each made of sections of 2-inch diameter, $\frac{1}{8}$-inch-wall aluminum tubing. The sections are independently supported, but are connected by sleeves to aid alignment. Each hoop section is clamped to 1-inch-diameter steel struts (see Fig. 4.3), which are in turn bolted to bosses welded to the tank wall. The 1280 PMT's viewing the main oil volume are mounted on the panels of the optical barrier.

The PSS is designed to be tolerant of irregularities in the shape of the tank wall and in the position and orientation of the bosses welded to it. After the tank was complete and the bosses welded on, the Fermilab Alignment Group surveyed the boss locations. As shown in Fig. 4.4, the survey indicated radial excursions on the order of an inch in the tank wall. We fabricated the struts in $\frac{1}{4}$-inch increments in length, with each boss assigned a strut length based on the survey. The base of each strut is a disk with a recessed ball-bearing at 


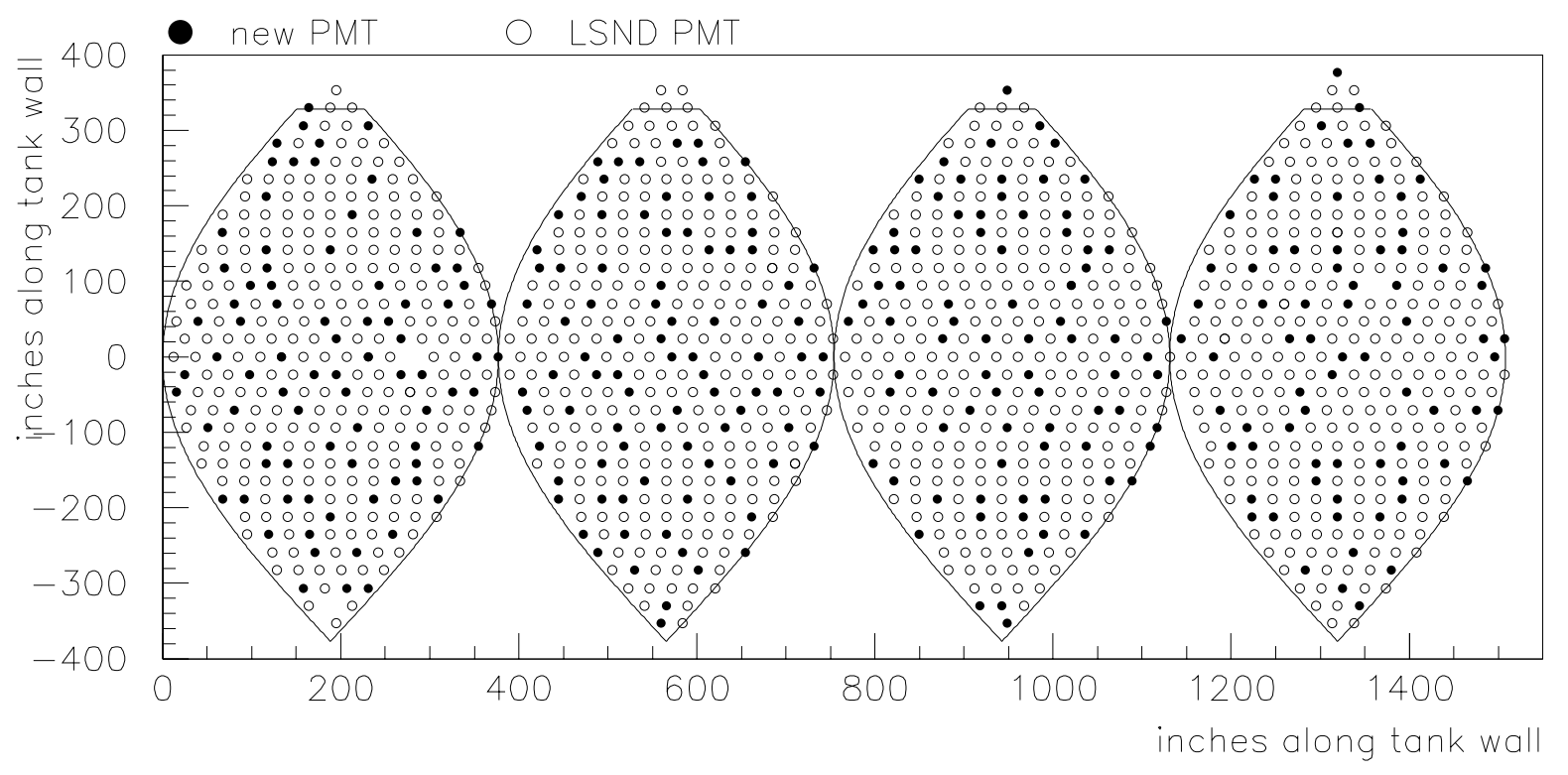

Figure 4.1: Main phototube layout. PMT's are not drawn to scale. There are a few gaps in this map where tubes had not yet been assigned. 


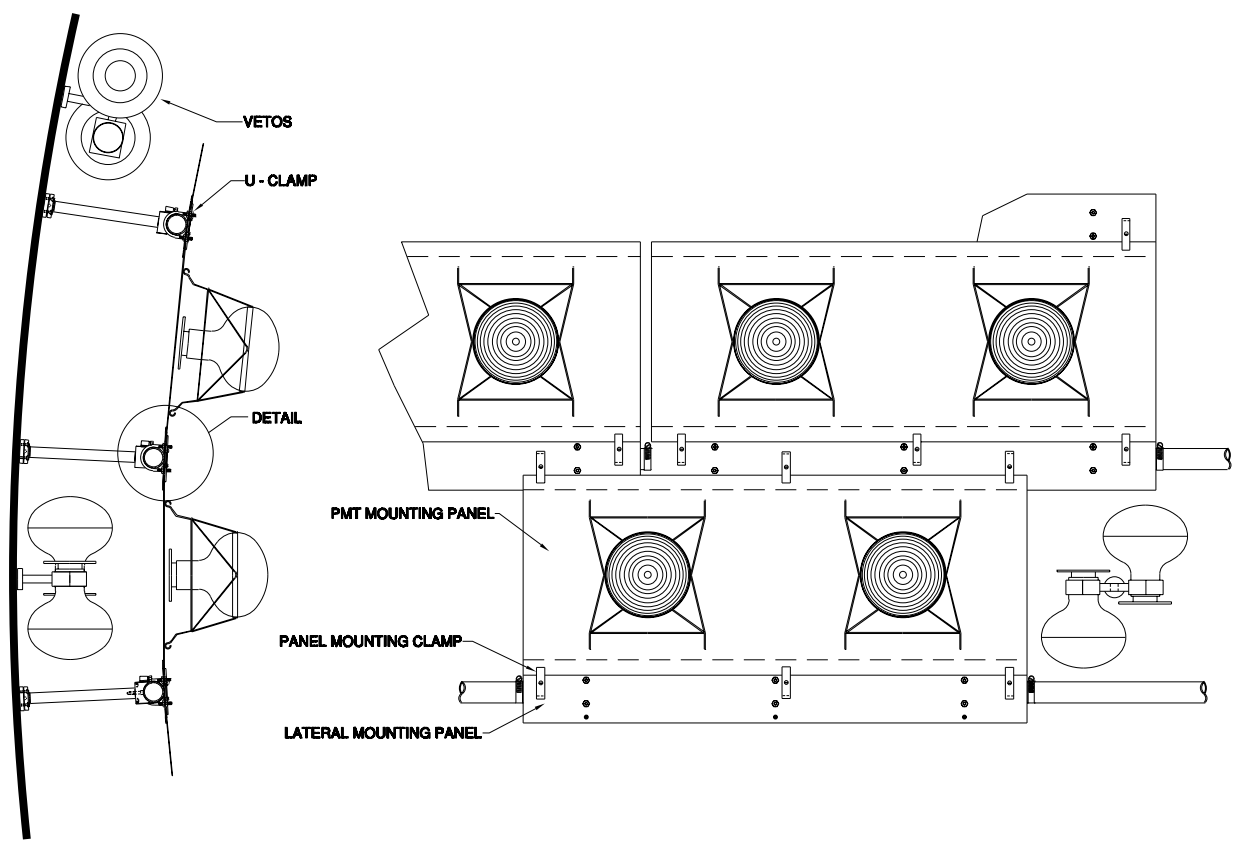

Figure 4.2: Overview of the Phototube Support System. Note: this figure shows an obsolete version of the veto cluster.

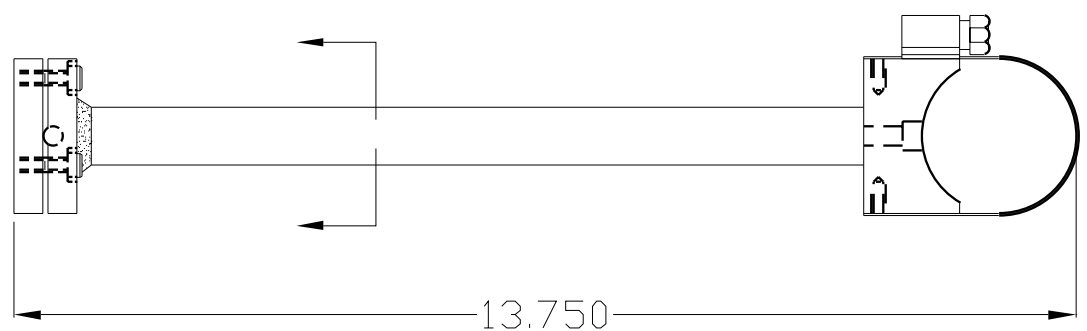

Figure 4.3: Struts with mounting hardware. The boss, welded to the tank wall, is on the left; the clamp for holding a lat section is on the right. 
the center. This provides a pivot point, and the relative tightening of the three mounting bolts can move the other end of the strut by about an inch in any direction. This provides further compensation for irregularities in the tank wall and allowed generous tolerances in the placement of the bosses on the tank wall, minimizing the cost of their installation.

The panels of the optical barrier are made from $\frac{1}{16}$-inch aluminum sheet. Each panel is approximately 4 feet wide by 2 feet high and holds two PMT's. We had anticipated rolling the panels to the correct curvature. However, experience with a full-scale prototype of a section of the PSS indicated that flat panels easily conformed to the lats, resulting in substantial cost savings. When mounted, each row of panels forms a section of a cone, with the whole optical barrier approximating a sphere. Figure 4.5 shows a typical panel. The panels do not mount directly to the lats. Instead, the panels mount to 6-inch-wide strips which in turn mount to the lats using U-bolts. The panels are attached to the strips by clips. The lower clips clamp the panels to the strips, while the upper clips capture the panels without clamping them. Gaps of about $1^{\prime \prime}$ are left between adjacent strips and panels in the same rows. These gaps are blocked by thin aluminum strips, pop-riveted to the strip or panel on one side. This arrangement has a number of virtues:

- The overlap provides a robust optical barrier.

- Variable gaps between panels horizontally and vertically allow loose mechanical tolerances, easing installation.

- The use of the strips and clips decouples the horizontal positions of panels in adjacent rows. This and the gaps mean that installation variations do not accumulate as rows are added.

- The fact that the panels are clamped only along one edge (and, similarly, the inserts connecting the lat pipe sections are only fastened on one end) allows the structure to shift slightly after installation.

It is also worth noting that, with the exception of the polar caps, each piece of the PSS can be carried by one person.

The ability of the sections of the structure to slide a little may be important when the tank is filled with oil. A finite element analysis of the tank showed that, while the displacements of the tank wall were, as expected, very small, differential motion of nearby struts could magnify this effect. The largest relative displacements, about $3 \mathrm{~mm}$, occurred between strut pairs that straddled the region where a tank leg joined the sphere. Because our ability to handle differential horizontal motion is more restricted, we modified the boss layout to have bosses centered horizontally on each leg (see Fig. 4.4), reducing the maximum relative horizontal displacement to less than $0.5 \mathrm{~mm}$. 


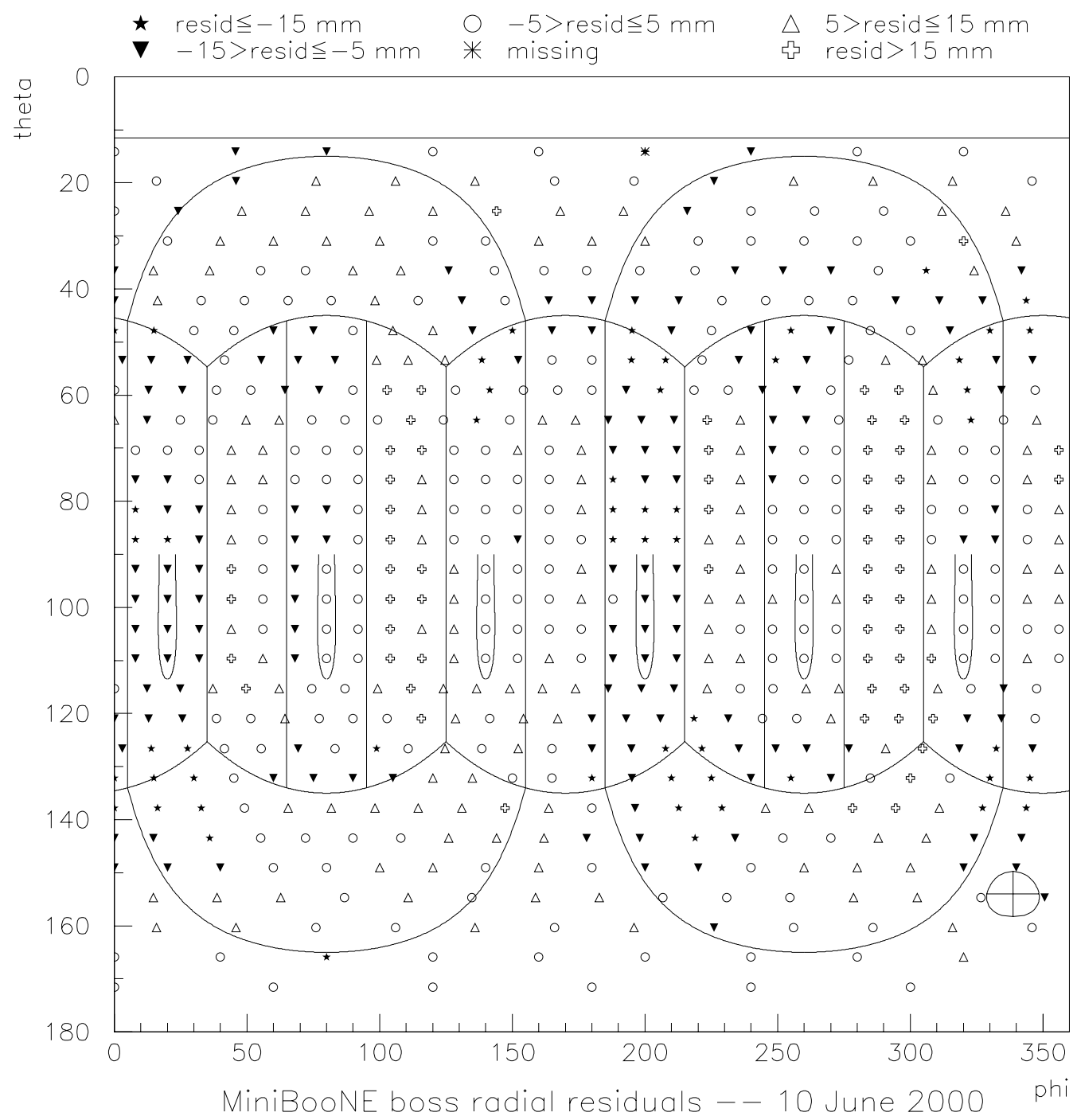

Figure 4.4: Survey of radial deviations of the tank from the best-fit sphere. Each main boss has been surveyed. In this cylindrical projection, the long lines are the edges of the panels forming the tank, the U-shaped lines are where the legs join the tank, and the squashed circle is the access port. 


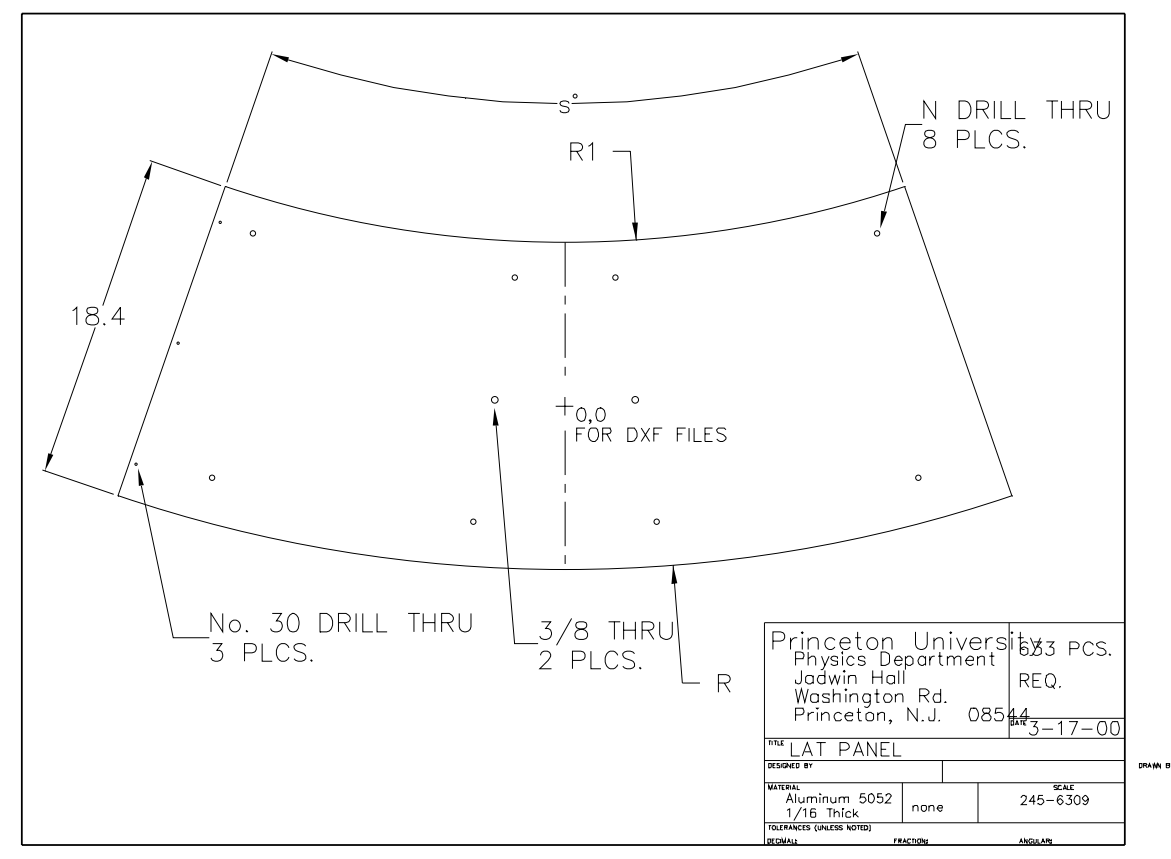

Figure 4.5: A typical panel of the optical barrier.

To ensure uniform distribution of the oil during filling and circulation, there are several ports through the optical barrier. These are equipped with baffles to prevent light from crossing the barrier.

Two PMT's are mounted to each panel using the existing PMT mounts from LSND (see Fig. 4.6). The majority of the LSND PMT's will be left in their mounts when they are removed from LSND. Some LSND tubes, to be used in the MiniBooNE veto, have to be removed from the stands. New tubes will be mounted in the stands thus freed up. The stands attach to blocks mounted on the panels. These blocks are designed to accomodate both existing sizes of LSND stand. When LSND was opened to harvest the phototubes, it was found that about 100 tubes had floated out of their stands. It was thus necessary to modify the support scheme. This is discussed in Sec. 4.6.

The veto PMT's are mounted in pairs on struts attached to bosses welded to the tank wall. Since the veto mounts were initially designed to use the same sticky foam tape that failed in the LSND mounts, a more secure scheme was 


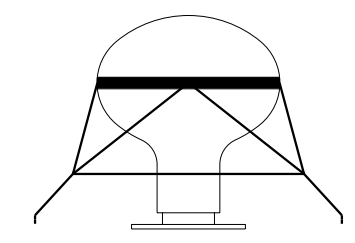

Figure 4.6: 8-inch photomultiplier in LSND stand.

developed. This scheme is shown in Fig. 4.7. Each phototube rests on a viton o-ring which in turn sits on a step in the end of an aluminum pipe. A cross of stainless steel wire captures the globe of the tube against the o-ring, while nylon screws center the neck of the tube in the pipe. The orientation of each cluster on its strut can be varied to avoid obstructions. Monte Carlo studies showed no differences in average light collection among various possible patterns in the orientation.

Tables 4.1 and 4.2 show the layout of main-tank and veto PMT's. The main PMT's are about $55 \mathrm{~cm}$ apart; the veto tubes are about $2 \mathrm{~m}$ apart. Table 4.3 gives the placement and dimensions of the latitudinal hoops. Table 4.4 shows the weight of various PSS components. These weights can be compared to the approximately 38 tons of the tank shell itself and the approximately 800 tons of oil.

To facilitate installation, the top and bottom "polar caps" will be treated specially. Each will hold the polar PMT, the next row of 6 PMT's, and, on the back, the polar veto cluster. The bottom cap will have to accomodate the coaxial fill pipe (see Sec. 3.5.3). The top cap will also hold the scintillator cubes of the calibration system (see Chapter 5). Each cap will be lowered into place as a unit, with the PMT's already mounted to it (see Sec. 4.7). Fig. 4.8 shows how the top polar cap is mounted. A white panel will be mounted just under the surface of the oil in the access portal to facilitate light collection in this region.

\subsection{Surface finishes}

Surfaces of the detector have been painted to provide high albedo in the veto volume and low albedo in the main volume. Reflection of light in the main volume of the detector can cause Cerenkov light to appear isotropic and delayed, degrading particle identification. We thus want surfaces in the main volume to be non-reflective. In the veto volume we simply want to maximize the total light collected by the sparse array of PMT's. Therefore surfaces in the veto volume are to be reflective. The inner surface of the tank wall, the bosses, the struts, 


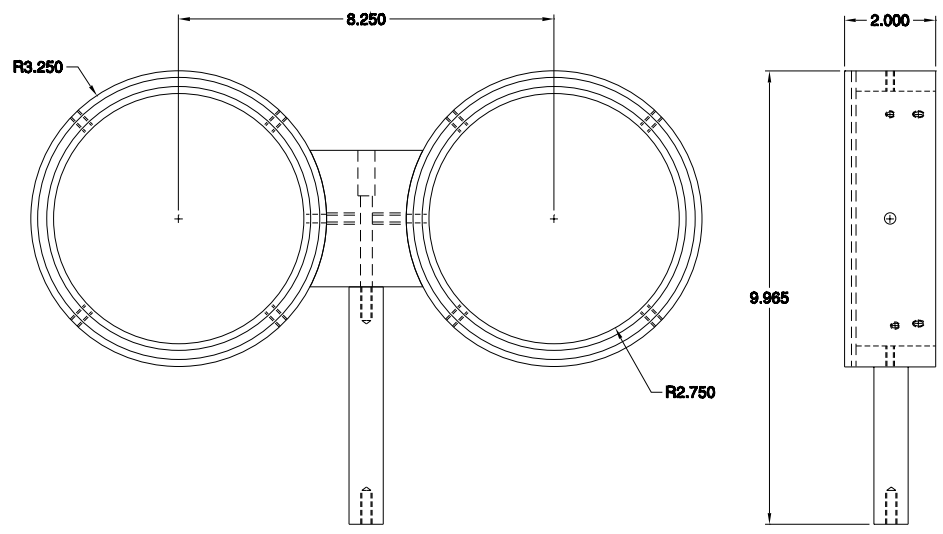

Figure 4.7: Veto PMT cluster. The strut mounts to a boss welded to the tank wall. The PMT's sit in the rings.

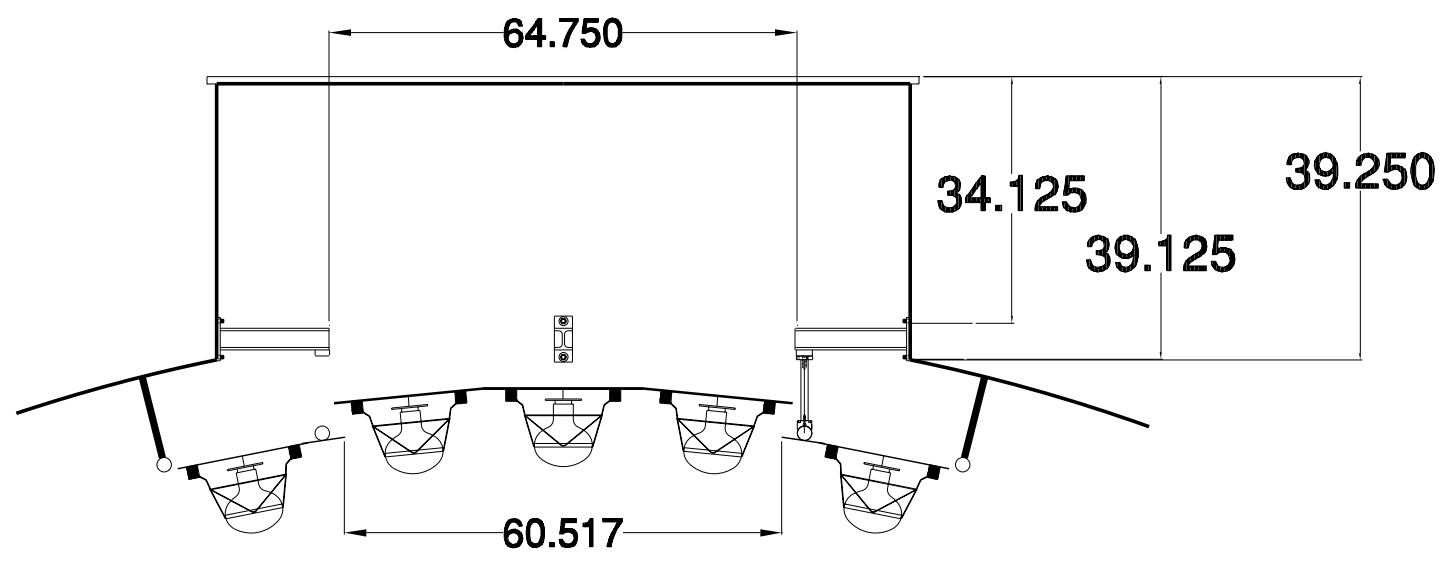

Figure 4.8: The top polar cap. 


\begin{tabular}{|r|r|r|r|r|}
\hline \hline Row & $\theta(\mathrm{deg})$ & Number of PMT's & $\Delta \phi(\mathrm{deg})$ & Spacing $(\mathrm{cm})$ \\
\hline \hline 1 & 0.000 & 1 & & \\
\hline 2 & 5.625 & 6 & 60.00 & 57.14 \\
\hline 3 & 11.250 & 12 & 30.00 & 56.87 \\
\hline 4 & 16.875 & 18 & 20.00 & 56.41 \\
\hline 5 & 22.500 & 24 & 15.00 & 55.77 \\
\hline 6 & 28.125 & 30 & 12.00 & 54.96 \\
\hline 7 & 33.750 & 36 & 10.00 & 53.98 \\
\hline 8 & 39.375 & 40 & 9.00 & 55.48 \\
\hline 9 & 45.000 & 44 & 8.18 & 56.21 \\
\hline 10 & 50.625 & 48 & 7.50 & 56.33 \\
\hline 11 & 56.250 & 52 & 6.92 & 55.93 \\
\hline 12 & 61.875 & 56 & 6.43 & 55.09 \\
\hline 13 & 67.500 & 58 & 6.21 & 55.72 \\
\hline 14 & 73.125 & 60 & 6.00 & 55.79 \\
\hline 15 & 78.750 & 62 & 5.81 & 55.33 \\
\hline 16 & 84.375 & 62 & 5.81 & 56.15 \\
\hline 17 & 90.000 & 62 & 5.81 & 56.42 \\
\hline 18 & 95.625 & 62 & 5.81 & 56.15 \\
\hline 19 & 101.250 & 62 & 5.81 & 55.33 \\
\hline 20 & 106.875 & 60 & 6.00 & 55.79 \\
\hline 21 & 112.500 & 58 & 6.21 & 55.72 \\
\hline 22 & 118.125 & 56 & 6.43 & 55.09 \\
\hline 23 & 123.750 & 52 & 6.92 & 55.93 \\
\hline 24 & 129.375 & 48 & 7.50 & 56.33 \\
\hline 25 & 135.000 & 44 & 8.18 & 56.21 \\
\hline 26 & 140.625 & 40 & 9.00 & 55.48 \\
\hline 27 & 146.250 & 36 & 10.00 & 53.98 \\
\hline 28 & 151.875 & 30 & 12.00 & 54.96 \\
\hline 29 & 157.500 & 24 & 15.00 & 55.77 \\
\hline 30 & 163.125 & 18 & 20.00 & 56.41 \\
\hline 31 & 168.750 & 30.00 & 56.87 \\
\hline 32 & 174.375 & 60.00 & 57.14 \\
\hline 33 & 180.000 & & & \\
\hline \hline & & & & \\
\hline \hline
\end{tabular}

Table 4.1: Layout of PMT's in the main tank. "Spacing" is the horizontal separation of the PMT's (measured from the centers of the globes) and can be compared to the row separation of $54.7 \mathrm{~cm}$. 


\begin{tabular}{|r|r|r|r|r|}
\hline \hline Row & $\theta(\mathrm{deg})$ & Number of PMT's & $\Delta \phi(\mathrm{deg})$ & Spacing $(\mathrm{cm})$ \\
\hline \hline 1 & 0.0 & 2 & & \\
\hline 2 & 18.0 & 12 & 60.00 & 193.22 \\
\hline 3 & 36.0 & 22 & 32.73 & 200.47 \\
\hline 4 & 54.0 & 30 & 24.00 & 202.35 \\
\hline 5 & 72.0 & 36 & 20.00 & 198.23 \\
\hline 6 & 90.0 & 36 & 20.00 & 208.43 \\
\hline 7 & 108.0 & 36 & 20.00 & 198.23 \\
\hline 8 & 126.0 & 30 & 24.00 & 202.35 \\
\hline 9 & 144.0 & 22 & 32.73 & 200.47 \\
\hline 10 & 162.0 & 12 & 60.00 & 193.22 \\
\hline 11 & 180.0 & 2 & & \\
\hline \hline
\end{tabular}

Table 4.2: Layout of PMT's in the veto. The tubes are mounted in pairs. "Spacing" is the horizontal separation of the PMT's (measured from the centers of the clusters) and can be compared to the row separation of $187.6 \mathrm{~cm}$.

\begin{tabular}{|r|r|r|r|}
\hline \hline Lat & $\theta(\mathrm{deg})$ & Diameter $(\mathrm{ft})$ & Circumference $(\mathrm{ft})$ \\
\hline \hline $1^{*}$ & 2.812 & 1.86 & 5.84 \\
\hline 2 & 8.438 & 5.56 & 17.46 \\
\hline 3 & 14.063 & 9.20 & 28.91 \\
\hline 4 & 19.688 & 12.76 & 40.08 \\
\hline 5 & 25.312 & 16.19 & 50.87 \\
\hline 6 & 30.937 & 19.47 & 61.16 \\
\hline 7 & 36.563 & 22.56 & 70.87 \\
\hline 8 & 42.188 & 25.43 & 79.90 \\
\hline 9 & 47.813 & 28.06 & 88.15 \\
\hline 10 & 53.438 & 30.42 & 95.56 \\
\hline 11 & 59.063 & 32.48 & 102.05 \\
\hline 12 & 64.688 & 34.23 & 107.55 \\
\hline 13 & 70.313 & 35.66 & 112.02 \\
\hline 14 & 75.938 & 36.74 & 115.41 \\
\hline 15 & 81.562 & 37.46 & 117.68 \\
\hline 16 & 87.188 & 37.82 & 118.83 \\
\hline \hline
\end{tabular}

Table 4.3: Location and dimensions of the latitudinal hoops. Only the top hemisphere is shown; the bottom is a mirror image of the top. ${ }^{*}$ The top lat doesn't actually exist: it is subsumed into the polar cap. 


\begin{tabular}{|c|c|}
\hline \hline Part & Total weight (tons) \\
\hline \hline panels/lat strips & 2.1 \\
\hline lats & 0.9 \\
\hline bosses/struts & 1.0 \\
\hline hardware & 0.05 \\
\hline PMTs/bases & 1.5 \\
\hline LSND stands & 0.3 \\
\hline cables (in tank) & 0.7 \\
\hline \hline Total & 6.6 \\
\hline \hline
\end{tabular}

Table 4.4: Total weight, in tons, of various PSS components.

the latitudinal hoops, and the outer side of the panels, strips, and overlaps of the optical barrier are painted white. The inner side of the optical barrier is painted black. Small parts on the inner surface of the optical barrier, such as the clips and PMT mounting blocks, are black-anodized.

We have measured the albedo of various paints in air using a tungsten lamp and integrating sphere. Figure 4.9 presents these measurements for several candidate white coatings. All of the measured coatings provide better than $80 \%$ albedo at wavelengths above $425 \mathrm{~nm}$. We use a conservative model of the measured albedo as a function of wavelength in our Monte Carlo simulations. The solid curve in Fig. 4.9 is the albedo published by Bicron for its paint. Of note is that our measurements at 436 and $545 \mathrm{~nm}$ confirm the published curve, but our measurement at $405 \mathrm{~nm}$ falls short. We have also measured albedos in oil by immersing a sample, a light source (an alpha emitter embedded in scintillator), and a detecting PMT. These in-oil measurements confirmed our rankings of candidate surface treatments.

The steel tank was painted after construction with Plasite 9060, a white epoxy coating. Plasite Protective Coatings (Green Bay, Wisconsin) markets this tank lining to the food and beverage industries, saying that it will not impart taste or odor, and that it meets FDA requirements for direct food contact. Our own tests indicated that it will not contaminate mineral oil (see below). The manufacturer's specifications for application of the coating included sandblasting the tank surface to "white metal," application of the coating to a dry film thickness of 12-14 mils in two coats, and elevated temperature curing.

The components of the phototube support structure were painted with coatings manufactured by Sherwin-Williams. We found that these coatings were non-contaminating. In albedo tests of samples gathered from various manufacturers, the S-W white offered the highest albedos, whereas the S-W black offered the lowest. The white is an aircraft paint (Jet Glo High Gloss) and the flat black is a military paint (camouflage chemical agent resistant coating). Application included a primer (the same primer for both aluminum and steel) 


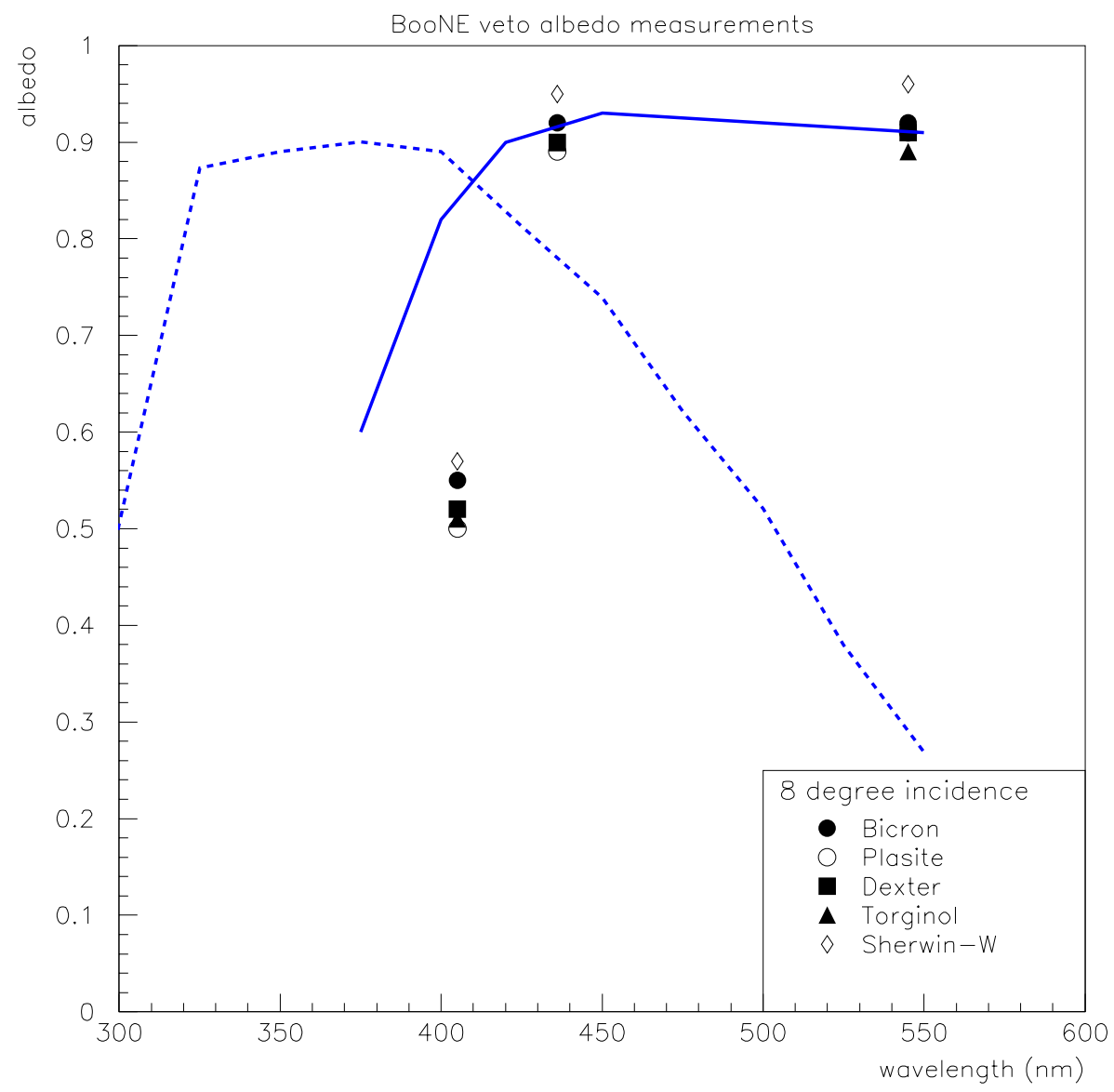

Figure 4.9: Albedo measurements for a variety of candidate white coatings. Also shown: the albedo curve published by Bicron for its paint (solid), and the phototube quantum efficiency in arbitrary units (dashed). 
followed by a single pass of topcoat for a total dry film thickness of about 3 mils and elevated-temperature curing.

Some of the smaller pieces of the PSS - panel clips, the blocks to which the PMT stands attach, calibration cubes - were black anodized. This process was shown to be non-contaminating, and is a fast, cost-effective way to finish a large number of small pieces. However, a smooth black anodized aluminum surface offers fairly high albedo at large incidence angles; to achieve lower albedo the surface must be scuffed (e.g., sandblasted) prior to anodizing. Anodizing was considered for the panels and rejected because of the need for low albedo over the full range of incidence angles and the concern that sandblasting might curl the panels. The smaller pieces were deburred in a tumbler, which left them slightly scuffed, making them ideal candidates for anodizing.

\subsection{Oil compatibility}

All materials in the tank will be immersed in mineral oil. We must be confident that these materials will not contaminate the mineral oil over the duration of the experiment. The materials and their potential for contaminating the mineral oil are:

- Painted surfaces: Of particular concern is the leaching of residual solvent from incompletely cured paint. Solvents contribute additional scintillating components to the mineral oil.

- Plastics: Plastic components, such as cable jackets, contain plasticizers, which can be the cause of contamination. A plasticizer is a material, usually an organic compound, that gets added to a polymer in order to produce a flexible plastic. Plasticizers tend to be volatile to some degree; for example, the fogging of the interior of car windshields is due primarily to the release of plasticizers from dashboards.

- Metals: The carbon steel tank will be painted, as will the aluminum components of the phototube support structure. Nevertheless, some metal may be exposed to the oil, through pores and nicks in the paint, and because some "buried" surfaces, like the inside of the latitudinal hoop tubing, will be difficult to paint. It is believed that metals do not cause contamination of the mineral oil directly, but their presence acts as a catalyst for oxidation, particularly at temperatures above $140^{\circ} \mathrm{F}$. Oxidation decreases the oil's attenuation length. Oxidation should not be a problem in MiniBooNE because the oil will be kept in a nitrogen environment at fairly low temperature, below $70^{\circ} \mathrm{F}$.

The surface coatings - the white of the veto volume and the black of the main volume - present the largest surface area of material in the oil and therefore present the greatest threat of contamination. This, along with evidence 
that the black paint used in LSND caused some problems, made us choose coatings for MiniBooNE carefully. (LSND experienced a rising level of scintillation over the approximately six years of its operation). The same care and testing procedures were applied to all candidate materials to be put into the tank.

Any material proposed for use in the tank was soaked in oil, usually for at least a week and often longer, with a sample at room temperature and another at $150^{\circ} \mathrm{F}$ to speed the aging process. The optical properties of the oil were then measured. A spectrophotometer is used to look for changes in light absorption, and a flourimeter is used to look for changes in scintillation properties.

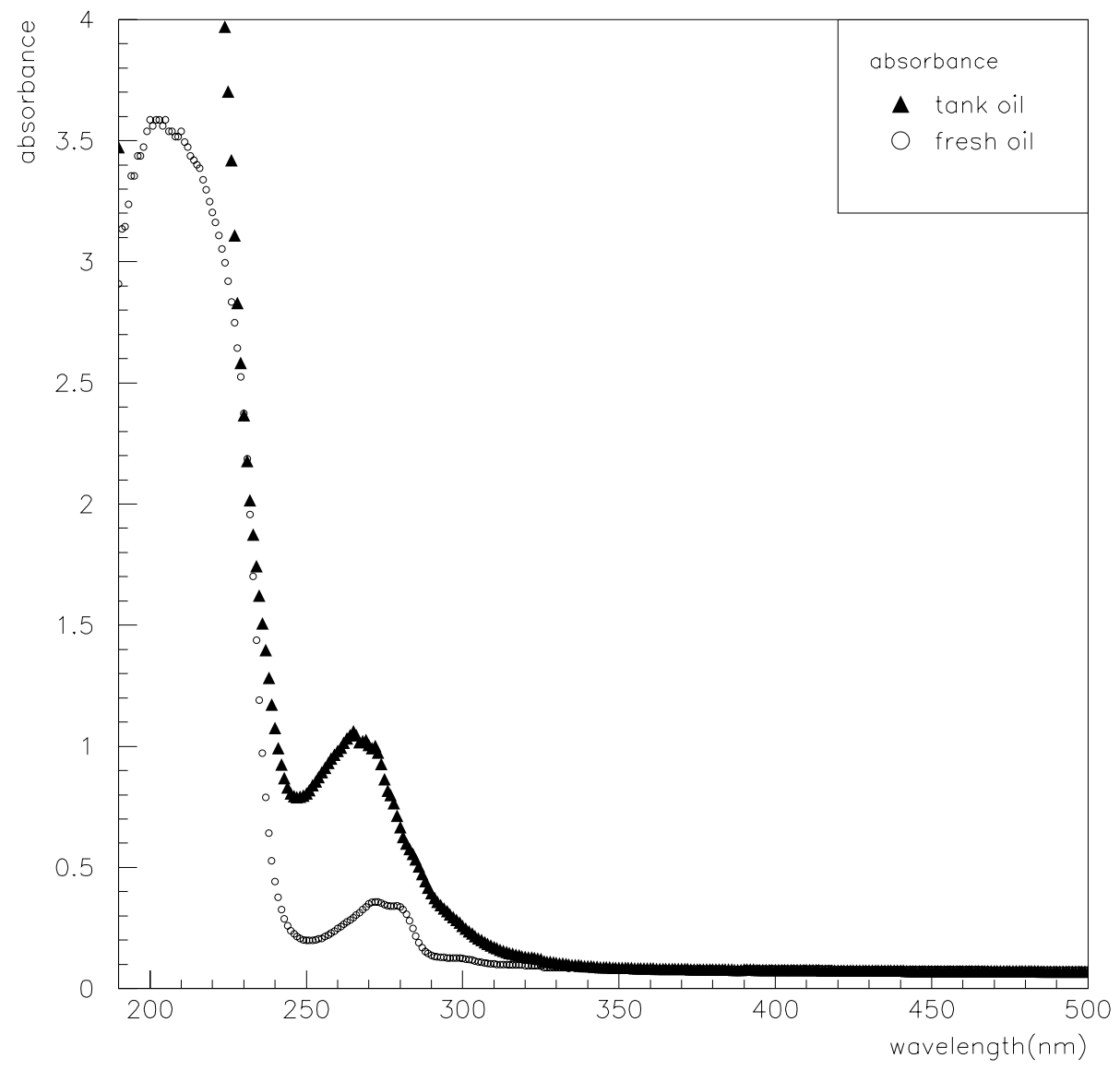

Figure 4.10: Absorbance spectra of fresh mineral oil from the Witco Corporation (circles) and oil from the LSND storage tank (triangles). 


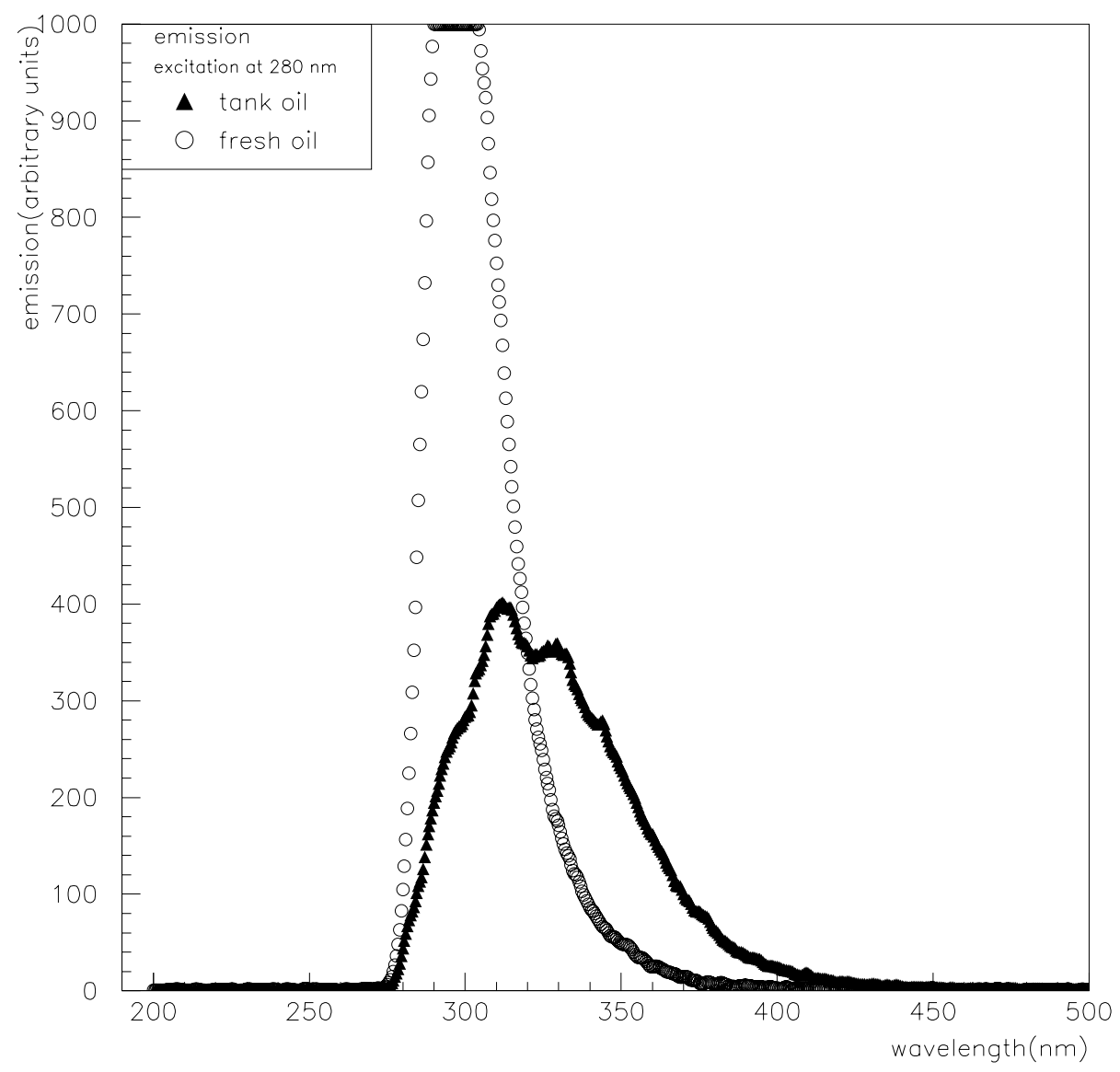

Figure 4.11: Emission spectra of fresh mineral oil from the Witco Corporation (circles) and oil from the LSND storage tank (triangles). The samples have been excited at $280 \mathrm{~nm}$.

Examples of these measurements are shown in Figs. 4.10 and 4.11 , which present the absorbance in $1 \mathrm{~cm}$ of oil (defined as $-\log _{10} T$, where $T$ is the transmission) and emission spectra, respectively, for a sample of recently acquired "fresh" WITCO mineral oil and for oil drawn from the LSND storage tank in 1998.

LSND has a 52,000 gallon oil storage tank that was prepared internally in the same way its the detector tank. In December 1992, oil was delivered to the LSND storage tank. In April 1993 50,000 gallons were transferred to the LSND detector, and subsequently b-PBD scintillator was added to the detector oil. 
Oil from the LSND storage tank therefore provides a sample of pure mineral oil from LSND. Whereas fresh mineral oil is odorless, the LSND storage tank oil has the smell of paint (which is also true for the oil from the LSND detector). Compared with the fresh oil, the storage tank oil absorbs significantly more light at 250 to $300 \mathrm{~nm}$. The emission spectra of Fig. 4.11 were produced after excitation at $280 \mathrm{~nm}$. The storage tank oil has a significantly broader excitation spectrum. Both of these measurements indicate the presence of contaminants in the storage tank oil.

The chosen tank and PSS coatings were found not to change the optical properties of the oil. In addition, we have confirmed that metals do not cause contamination of the oil at room temperature and that they seem to catalyze oxidation at higher temperature. Our tests with aluminum and copper in oil at $150^{\circ} \mathrm{F}$ indicate contamination (the copper is worse), and the contamination at $150^{\circ} \mathrm{F}$ is much smaller when the samples are kept in a nitrogen environment. Therefore unpainted metal is acceptable in MiniBooNE (we will have some unpainted aluminum, but no copper), because the oil will be cool and under nitrogen.

We found that PVC-jacketed cables, including the RG-58C/U with a "noncontaminating" jacket used in LSND, contaminate mineral oil. An example of these results is given in Fig. 4.12, which shows absorbance for oil in which new samples of PVC-jacketed RG58 were soaked for one week at room temperature. Further tests indicate that as the cable soaks its contamination rate decreases (the rate at which material leaches from the cable decreases). The bottom of Fig. 4.12 shows absorbance when the same cables are soaked again for one week in fresh oil. The top of Fig. 4.13 shows absorbance for oil in which a sample of cable from LSND (i.e., the cable was immersed in the LSND tank during the life of the LSND experiment) soaked for one week at room temperature.

Teflon-jacketed cables were found to be non-contaminating and were chosen as a feasible alternative to PVC. The bottom of Fig. 4.13 shows absorbance for oils in which teflon-jacketed cables soaked at $150^{\circ} \mathrm{F}$. One of the samples shown was allowed to soak for over two months, with no contamination observed.

The bases and necks of the LSND phototubes were coated in Hysol potting compound. New Hysol samples and samples taken from an LSND phototube were found to contaminate oil. Therefore we chose a new encapsulant, epoxy EP21LV from Master Bond (Hackensack, New Jersey). This epoxy meets FDA requirements for food compatibility and was found to be non-contaminating in our tests. Both MiniBooNE's old and new phototubes will be potted in Master Bond epoxy - in the case of the old tubes, the Master Bond will go right over the Hysol. 

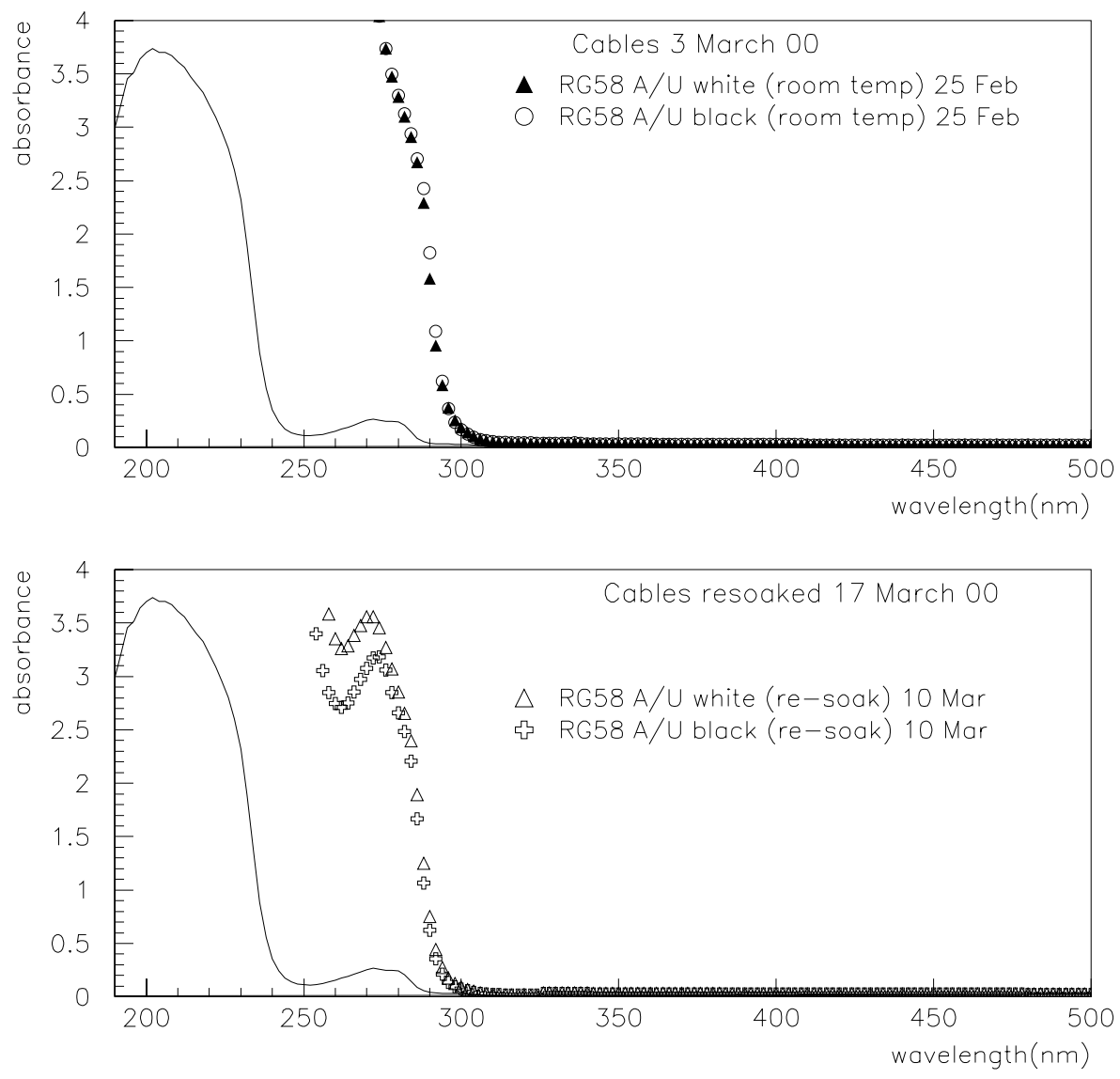

Figure 4.12: Absorbance spectra for oil in which new samples of PVC-jacketed cable soaked. Top, black and white RG58 began to soak at room temperature on 25 Feb 2000 and the oil absorbance was measured on 3 March. Bottom, the same cables were put into fresh oil on 10 March and the oil absorbance was measured on 17 March. The solid line is the control spectrum for oil in which nothing soaked. 

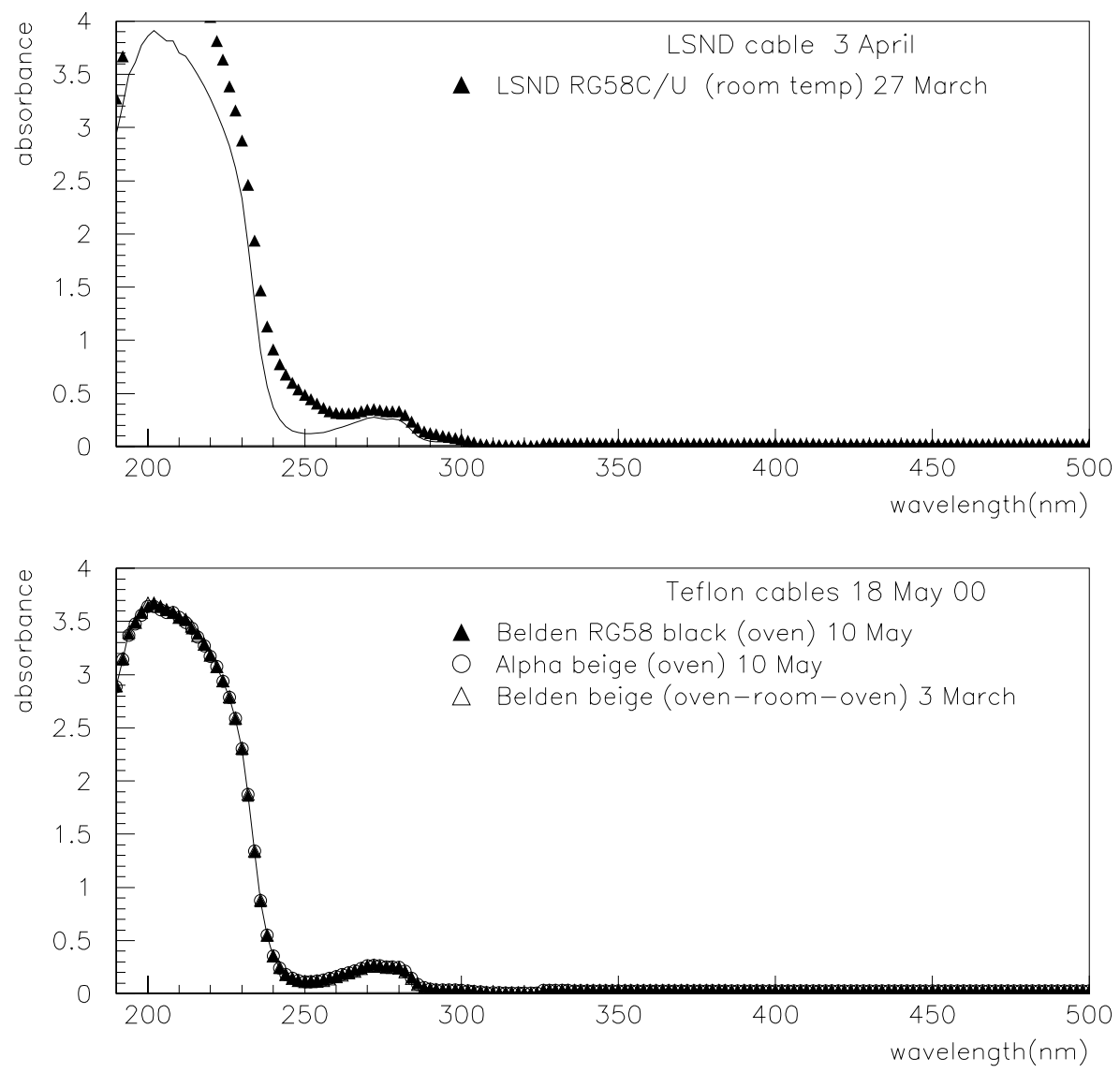

Figure 4.13: Absorbance spectra. Top, a sample of cable from LSND (i.e. a piece of cable that was immersed in the LSND tank during the duration of the LSND experiment) began to soak on 27 March 2000 and the oil absorbance was measured on 3 April. Bottom, oil absorbance measurements for oil samples exposed to teflon-jacketed cables at $150^{\circ} \mathrm{F}$. 


\subsection{In-tank cable plant}

We describe here the cable runs to and from the in-tank components, primarily from a mechanical point of view. The remaining cabling is described in Secs. 3.5.4 and 6.8; all electrical considerations are described in the electronics section, Chapter 6 . We concentrate on the connections to the 8-inch photomultiplier tubes. Where they differ, connections to the various calibration elements are discussed with the calibration systems themselves.

The in-tank cable run links the photomultiplier tubes to the preamplifiers, located in crates in the tank access area next to the tank access port. A single RG-58 cable both supplies high voltage to a PMT and carries the signal from PMT to preamp. As described in the previous section, we use a teflon-jacketed cable, Belden 88240 , for compatibility with the mineral oil. This cable has the further advantage of being plenum-rated. It is thus suited for the run under the computer floor to the preamps. As most of the run is in the veto section of the tank, we use cable with a white jacket. The cable terminates at the preamp end in a SHV jack which mounts into a multi-connector block. The run from this connector is down the preamp rack and under the floor of the tank access area to the access portal of the tank.

The penetration of the tank wall is through airtight feedthroughs bolted to the flange on the tank's access portal (Sec. 3.2.4). The oil level in the access portal remains below these flanges - the seal is to maintain the nitrogen atmosphere in the tank. Each flange accepts four bundles of 98 cables. For mechanical reasons, each bundle is divided into two 49-cable sub-bundles, each with its own feedthrough. A feedthrough and the panel on which it mounts to a tank flange are shown in Fig. 4.14. The feedthrough is made of two aluminum plates with holes for the 49 cables. Captured between the plates on each cable is an o-ring, making a gas-tight seal.

To maintain uniformity in pulse shape from tube to tube, cables of a fixed length, 100 feet, were used for almost all channels, despite the disparity in the length of the cable runs to the top and bottom of the tank. (As a hedge, a few of the cables with the longest runs were cut to 110 feet. The excess can be removed if 100 feet suffices.) Since there is a lot of excess cable, and we do not want excessive slack that will block light collection in the tank, the each cable is captured in the feedthrough with only the length necessary to reach the PMT (with extra slack for splicing and rounded up to the next 5-foot increment) extending from the inner side. The bulk of the slack is thus gathered under the computer floor in front of the preamp racks.

The bundles were all fabricated in advance: the cables were cut to length, threaded through the feedthrough, captured with the appropriate lengths on either side, and labelled, and the SHV connector mounted and tested. Each bundle was then passed through the port in the tank as a unit. Once through the port, each bundle was strain-relieved at the bottom of the access portal in a heavy bar mounted on studs welded to the tank. From this corner, the cable 

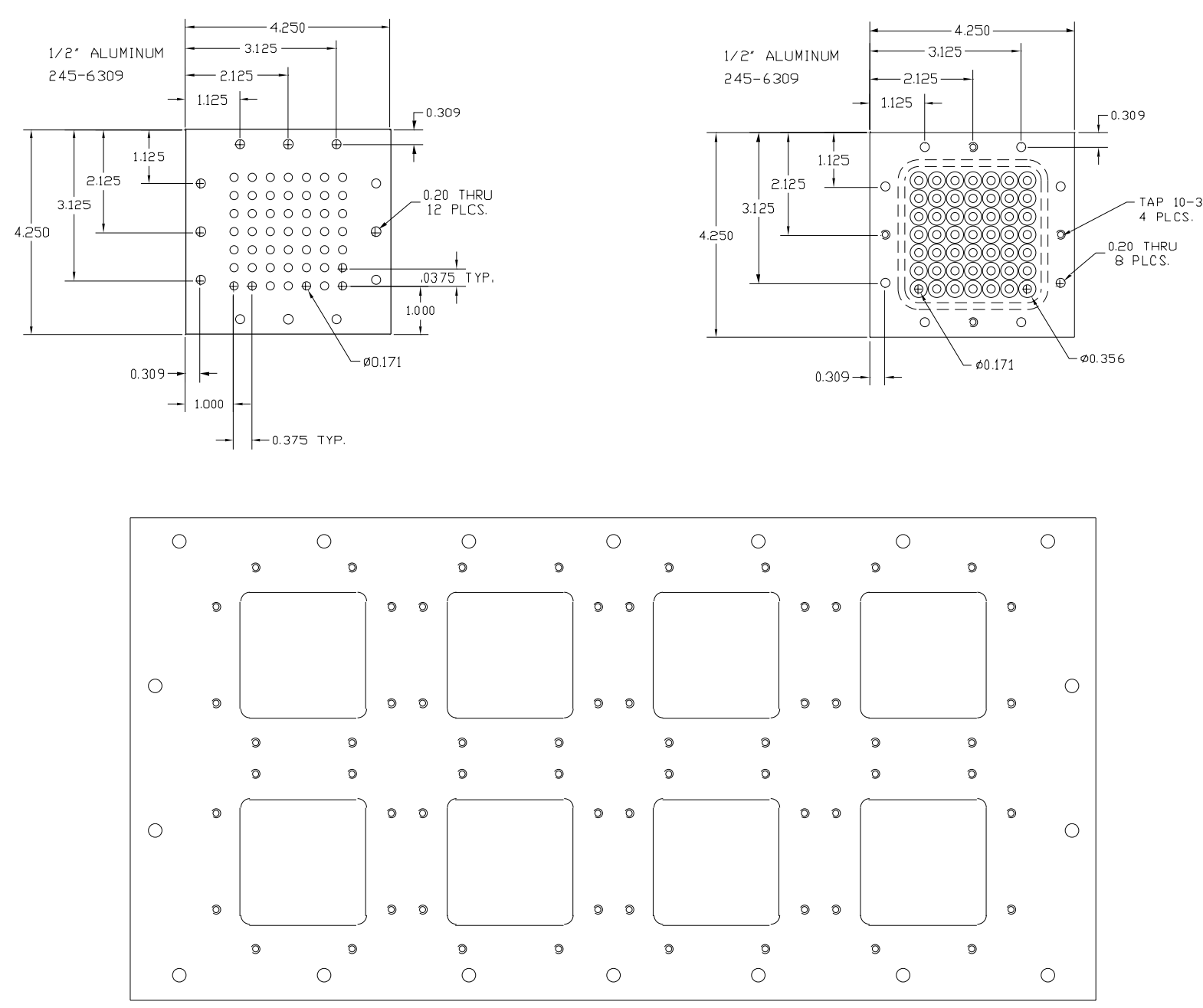

Figure 4.14: Cable feedthrough and mounting panel. 
bundles run down the tank wall, held in place by steel straps spanning pairs of studs on the wall. Each cable bundle serves the main and veto PMT's in a vertical slice of the tank from top to bottom. At a horizontal row of main or veto PMT's, cables are peeled from the nearest bundle and routed to each PMT. Cables for the main PMT's cross the veto region and penetrate the optical barrier through pairs of holes near the center of each panel. We simply let each cable run from the tank wall directly to its hole in the optical barrier, except where this path would drape the cable directly in front of a veto PMT. After passing through the optical barrier, the cable is spliced to the 5-foot piece of cable that is permanently attached to the PMT base. The splice is made with a coaxial splice kit (Raychem B-202-81, formerly D-150-0071). This system was used in LSND and found to be very reliable. To seal out oil, the splice is covered with a length of $\frac{3}{8}$ " teflon shrinktube (SPC Technology SST-024), which shrinks onto o-rings on either side of the splice.

As each splice is made, it is tested. Prior to splicing, a pulser is connected to the cable at the preamp end and the reflection (at about $370 \mathrm{~ns}$ ) is observed on the scope. Since the bases are back-terminated, the disappearance of the reflection indicates both that the splice is good and that the labels at the two ends of the cable match.

\subsection{PMT preparation}

After the PMT's have the bases attached, are tested, and are assigned to a location in the tank, they are prepared for installation. This process consists of these steps for each tube:

- It is washed in a mild solution of detergent and distilled water, then rinsed in clean distilled water, then allowed to dry for 24 hours. The main purpose is to remove the scintillator-doped oil residue from LSND.

- New tubes are mounted in stands. Old tubes destined for the main tank are re-mounted in stands. The centering clips that hold the neck of the tube are not installed until after dipping.

- It is dipped in black Master Bond EP21LV encapsulant from the base up to the bottom of the globe. This is to protect the oil from the components of the base and vice versa and may also serve to attenuate any light generated by breakdown in the dynode structure of the tube. Old tubes were already coated in black Hysol. This was found to contaminate mineral oil (Sec. 4.4), so the old tubes are also dipped in Master Bond.

- Veto tubes are removed from their stands and mounted in veto mounts.

- Main tubes have the centering clips attached around the tube neck. A stripe of Master Bond is run down the neck of the tube over the silicone rubber 
cushion on each centering clip. This attachment serves as a mechanical backup in case the metal band and foam tape at the tube's equator slip, as they occasionally did in LSND.

This work is done in a cleanroom. It was found that maintaining low humidity was necessary for the proper curing of the Master Bond.

\subsection{Installation}

The installation of the PSS begins with the tank plant in this state:

- The tank is complete, the bosses and other mounting features are welded in place.

- The tank has been painted with the final veto-white paint and is free from debris and clean.

- The buildings enclosing the tank access are in place as are facilities to keep the tank and a modest-sized staging area in the enclosure clean.

- The hoist is installed in the tank access area.

An intricate scaffolding system is required for the installation of the apparatus in the tank. The system must allow access to virtually the entire inner surface of the tank to allow cabling, veto installation, and attachments to the preinstalled bosses. The scaffold must also have an area where work can be done up to approximately 30 inches from the tank wall for installation of lats, optical barrier panels, and PMT's.

The scaffolding was supplied and installed by Bartlett Services, Inc. It consists of six levels with an internal stairwell. The structure is supported by legs and diagonal braces that rest against the tank wall. The painted surface of the tank is protected by rubber pads. Work is done on the top level, which is then lowered or removed to allow access further down the tank wall. The working level extends horizontally to within a few inches of the tank wall, providing a safe, stable work space.

Personnel access to and from the tank is via the 36-inch access port ("manhole") near the bottom of the tank. Light tools and parts are also brought into and removed from the tank via this pathway, which consists of a spiral stairway down the side of the vault and a ladder through the manhole that gives access to the bottom level of the scaffolding. A small temporary clean room surrounds the access port. Heavier objects are lowered through the top access portal using the electric hoist. Ventilation is maintained by blowers delivering air through the manhole and withdrawing it from the top portal. The experience of tank construction companies shows this to be completely adequate. 
With the scaffolding in the tank, the cable bundles were installed as described in Sec. 4.5. Installation of the PSS itself begins near the top, starting with the top lat. We work down from there, with each cycle of installation ending when the top remaining deck of the scaffolding is reached. The strategy is to install as much hardware as possible at each level before any PMT's are mounted.

The first steps at each level involve installing the lat pipes to the desired height using a rotating-laser level that traces a horizontal line around the tank. The height reference is a measuring tape hanging from the top access portal. It was calibrated by using the laser level to transfer the survey coordinates of many bosses to the tape and averaging.

One installation cycle proceeds in these steps:

- Peel out the cables needed from the bundles on the tank wall.

- Install latitudinal hoops as far down as the scaffolding allows.

- Attach struts to bosses on tank wall. Each strut has an assigned length.

- Set the rotating-laser optical level to the desired lat height.

- Adjust strut base screws to pivot the clamp end of each strut to the laser. A fixture that attaches to the clamp end has a scribe mark where the center of the lat will be.

- Install latitudinal hoop sections and their linking sleeves.

- Measure several diameters of the hoop and compare the height to the previous lat with a measuring jig.

We then go back up to the top bare lat and work down again, installing the optical barrier and rows of main and veto PMT's. The order of these operations depends somewhat on convenience of reach.

- Mount lat strips on lats using U-bolts.

- Rivet overlap panels to strips.

- Bolt panel clips to rivet nuts on strips.

- Install a row of veto clusters if there will be one behind this row of panels.

- Attach veto strut to boss.

- Mount veto cluster on strut.

- Orient the cluster on the strut to 1) give clearance from lat pipes, 2) keep photocathodes as far from steel struts as possible, and 3) minimize the hardware shadowing the tubes.

- Splice cables.

○ Test splices (see Sec. 4.5). 
- Mount a row of optical barrier panels.

- Locate the first panel to provide the correct "clocking" of this row.

- Attach panels to upper and lower lat strips using clips.

- Adjust gaps between panels to give uniform PMT spacing.

- Rivet overlap panels to main panels.

- Run cables across veto and through holes in panel.

- Install a row of main PMT's, two per panel.

- Bolt mounting blocks to rivet nuts in panels.

- Attach stands holding PMT's to mounting blocks.

- Splice cables.

- Test splices (see Sec. 4.5).

- Feed cable back through hole in panel till the splice rests against the panel. This leaves the white cable in the veto and the black cable, attached to the base, in the main tank.) Coil slack in black cable and tie to stand.

- Lower or remove the top deck of scaffolding.

In practice, each cycle allows the installation of 2-3 rows of main tubes and takes approximately two weeks. We estimate that the PMT's end up placed with respect to their nominal positions within $\frac{1}{2}^{\prime \prime}$ radially, $\frac{1}{2}^{\prime \prime}$ in height, and $\frac{1}{2}$ " horizontally within a row. The "clocking" precision of a whole row is approximately $1-2^{\prime \prime}$.

At regular intervals, the tank ports are closed and the installed PMT's and cabling are tested at high voltage using a temporarily-installed flasher system.

After the installation reaches the manhole (offset approximately 8 feet horizontally from the bottom of the tank), the installation of several panels will be skipped to allow continued access. At this point, work on the top polar cap and the bottom of the tank will go in parallel to facilitate installation of the calibration equipment (see next chapter) that hangs in the tank. The PMT's at the bottom of the tank will be installed onto the bottom polar cap outside of the tank. This cap will be lowered into place as a unit and the coaxial fill pipe installed. The calibration flasks will be hung from their wires and their hanging wires fastened to the optical barrier at the bottom. When this step is complete, the top polar cap with its PMT's and calibration cubes will be lowered into place.

Finally, we will work toward the manhole, installing the skipped panels and PMT's. The top light reflector will be installed just below the nominal oil level, a white panel will be installed in the manhole trunk, and the access portal hatches will be sealed. 


\section{Chapter 5}

\section{Calibration Systems}

\section{$5.1 \quad$ Introduction}

The calibration systems are designed to (1) provide information on the PMT response that is needed as input for the event reconstruction and particle identification calculations, (2) calibrate the position, energy, and direction determination of the reconstruction program using stopping cosmic ray muons and Michel electrons from muon decay and (3) measure the attenuation of light by the oil in the detector tank.

The cosmic ray muon calibration system will consist of a muon tracker above the detector and scintillator cubes inside the detector. The muon tracker will give the direction of cosmic-ray muons while the cubes will give a precise position measurement of stopping muons and decay electrons.

The laser calibration system will provide short pulses of light from a tunable dye laser to 4 flasks at various locations in the detector. The system will be very similar to the system used successfully in LSND. It will be used to determine phototube time-offsets and gains and to determine time-slewing corrections.

The oil monitoring system will measure attenuation of light as a function of wavelength in the detector. This system will also measure reflectance properties of PMTs and of the optical barrier between the veto region and the main detector region.

\subsection{The Cosmic Ray Muon Calibration System}

The cosmic ray muon calibration system will use through-going muons as well as stopping muons and their decay electrons to calibrate the event reconstruction program. This calibration system will provide a precision calibration of the energy, direction and position of muons for the complete range of muon energies (100 MeV to $1 \mathrm{GeV}$ ) of interest in the experiment. It will also provide 
a calibration using $50 \mathrm{MeV}$ electrons from the observed Michel electron energy spectrum. A muon tracker located above the detector will be used to determine the entering positions and directions of muons that stop in the detector. The stopping positions can be determined from the locations of the decay electrons. The muon energy can then be obtained from the muon range with an uncertainty due to range straggling of approximately $3 \%$. The determination of muon energy from range is a well established technique in this energy region.

Six scintillation cubes described below will be used to obtain samples of stopping muons and decay electrons for which the absolute stopping positions are known with uncertainties of less than $3 \mathrm{~cm}$. With a prototype cube we have measured a rate of 1900 stopped muons per month at sea level. We estimate approximately 1300 muons will stop in a cube per month in the detector and 600 of these will have clean muon and electron signals separated by more than $1 \mu \mathrm{s}$. These events will be obtained by triggering on a coincidence of a cube signal with a minimum size detector signal. This trigger will add approximately $1.0 \mathrm{~Hz}$ to the normal trigger rate. For these samples the uncertainties in the muon energies will arise almost entirely from range straggling. The systematics can be kept small and, by averaging over events, we should be able to obtain an absolute energy calibration between $1 \%$ and $2 \%$ for $E_{\mu}>200 \mathrm{MeV}$. In addition, these cubes will provide an absolute calibration of the positions obtained with the event reconstruction program at these six locations.

It is important to have some calibration over the whole detector in addition to having precise calibrations at selected locations. Therefore, we will analyze a large sample of muons which traverse the muon tracker and then either pass completely through the detector or stop in the detector. This calibration sample will be obtained with a trigger from a coincidence of a muon tracker signal and a minimum size detector signal. We have simulated and reconstructed a large sample of such muons. We then studied how the tracker information could be used to calibrate the detector with these muons. The dimensions of the tracker scintillators are based on these studies.

\subsection{Muon Tracker}

The muon tracker is divided into 2 sets of 2 layers of scintillator hodoscope and will provide two sets of coordinates from which the direction will be determined. The top layers will be just below the ceiling of the tank access area and the bottom layers just above the hatch sealing the access portal of the tank as shown in Fig. 5.1. Where feasible we plan to use materials and components from previous experiments. The PMTs and bases (RCA 6342A) will come from the CCFR experiment. Figure 5.2 shows the PMT base diagram. Eight existing sheets of scintillators, $5^{\prime} \times 5^{\prime} \times 1^{\prime \prime}$, will be cut to make the 104 pieces needed in the tracker. We also plan to use Fermilab PREP electronics.

Each of the two layers at the ceiling will have 23 strips $10 \mathrm{~cm}$ wide and 7.5 


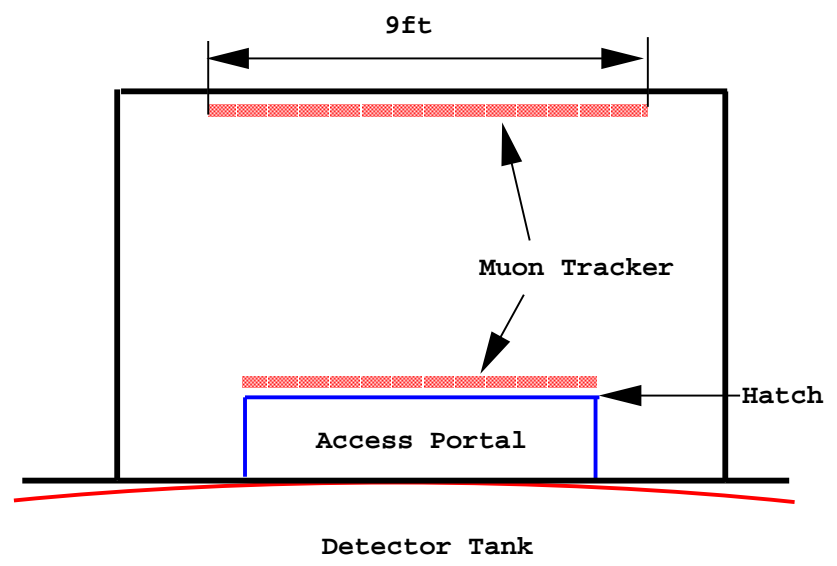

Figure 5.1: Muon tracker

feet long. There will be 46 PMTs for each layer (two per strip). With PMTs and bases these layers will cover an area of $9^{\prime} \times 9^{\prime}$ on the ceiling. The total weight of scintillator is $1100 \mathrm{lb}$. The support structure will be built into the ceiling and the walls of the access area above the tank.

Each of the two layers resting on the access portal will have 28 strips $6 \mathrm{~cm}$ wide and $170 \mathrm{~cm}$ long. There will be only one PMT per strip. With PMTs and bases these layers will cover an area of $193 \mathrm{~cm} \times 193 \mathrm{~cm}$. An aluminum support structure will hold these scintillators. For ease of assembly and removal each layer will consist of two mechanical subunits bolted together. Each subunit will hold 14 strips, weigh $100 \mathrm{lb}$ and have a size of $85 \mathrm{~cm} \times 193 \mathrm{~cm}$. Thus if access is needed to the access portal it will be relatively easy for two people to remove these subunits.

For the top two planes the signals from the 2 PMTs on each strip will be added passively and amplified if necessary. The PMT signals will then run to discriminators in the electronic crates. There will be 102 discriminator channels, 23 for each top plane and 28 for each bottom plane. An "or" of all the strips in each plane will be formed using logic fan ins and a coincidence of the 4 planes formed with a logic unit.

One QT electronics board will be modified so that it can record the status of the discriminators for the 102 scintillation strips for each event.

\subsection{Scintillation Cubes}

The six cubes are included in order to obtain precise position measurements of stopped muons and electrons in the tank. Although they are quite small compared to the tank itself, 600 muons are expected to stop in each cube every 


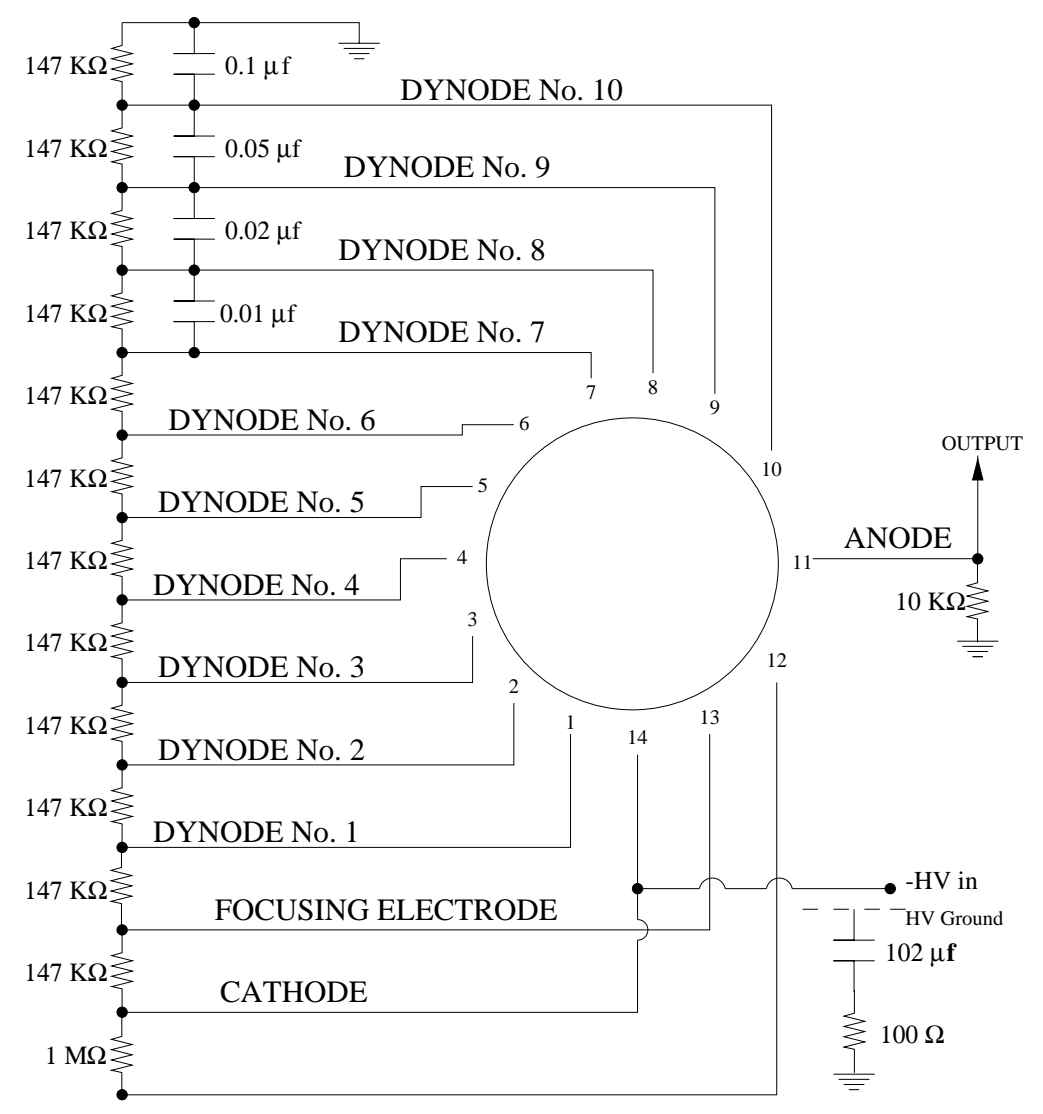

Figure 5.2: PMT Base Configurations. 


\begin{tabular}{|r|r|r|r|r|}
\hline $\operatorname{depth}(\mathrm{cm})$ & $\phi$ & $x$ & $y$ & $z$ \\
\hline 30 & $0^{\circ}$ & 0 & 545 & 60 \\
60 & $60^{\circ}$ & 52 & 515 & 30 \\
100 & $120^{\circ}$ & 52 & 475 & -30 \\
200 & $180^{\circ}$ & 0 & 375 & -60 \\
300 & $240^{\circ}$ & -52 & 275 & -30 \\
400 & $300^{\circ}$ & -52 & 175 & 30 \\
\hline
\end{tabular}

Table 5.1: Cube positions are listed in this table. The radius of the spiral is 60 $\mathrm{cm}$, the radius of the optical barrier is the tank radius $(610 \mathrm{~cm})$ minus the veto $\operatorname{depth}(35 \mathrm{~cm})$. Therefore, $x=60 \sin (\phi), y=610-35-\operatorname{depth}, z=60 \cos (\phi)$.

month. An optical fiber is used to transport light from a cube to a PMT located just outside the access portal. The cubes and fibers will be in leak-tight containers and optically isolated from the detector.

The cubes will be 2 inches on a side and will be located under the muon tracker. They will be $60 \mathrm{~cm}$ away from the $y$-axis (the center axis) and positioned in a descending spiral with a $60^{\circ}$ separation in the azimuth. They will be located at $30 \mathrm{~cm}, 60 \mathrm{~cm}, 100 \mathrm{~cm}, 200 \mathrm{~cm}, 300 \mathrm{~cm}$, and $400 \mathrm{~cm}$ below the optical divider. The cube positions are listed in Table 5.1.

A cube is composed of two pieces of Bicron BC408 scintillator, each $2^{\prime \prime} \times$ $2^{\prime \prime} \times 1^{\prime \prime}$. The two pieces of scintillator are each grooved down the center of one face. The grooves on the two pieces are aligned to create a $4 \mathrm{~mm}$ diameter hole through the center of the cube. A green wavelength-shifting fiber, Bicron BCF$91 \mathrm{~A}$, is inserted in the hole. It absorbs and reemits scintillation light, some of which travels along the fiber. This light is then transported to the PMT using a clear $4 \mathrm{~mm}$ fiber, BCF-98, glued to one end of the green fiber. The other end of the green fiber is flush with the surface of the cube and has a mirror glued to it to reflect light back along the fiber towards the PMT. The cube is wrapped in aluminized mylar. Multiclad versions of both fibers, BCF-91A and BCF-98, will be used because they provide $50 \%$ more light than the standard versions. Bicron BCF-98 is used to transport the light because it has a longer attenuation length $(\sim 6 \mathrm{~m})$ than BCF-91A. The fibers will travel from the cubes up into the access portal and then through a leak-tight feed-through panel on the side of the access portal and under the false floor in the access area. The PMTs will be located in a box under the floor near the feed-through panel.

Figure 5.3 shows the setup used to test a prototype cube with a $1.5 \mathrm{~m}$ fiber. Small counters above and below the cube were used to trigger on cosmic ray muons traversing the cube. Figure 5.4 shows the pulse spectrum obtained with this setup. There are an average of 45 photoelectrons per cosmic ray muon.

Each PMT output will be amplified and then split into two signals, one of 


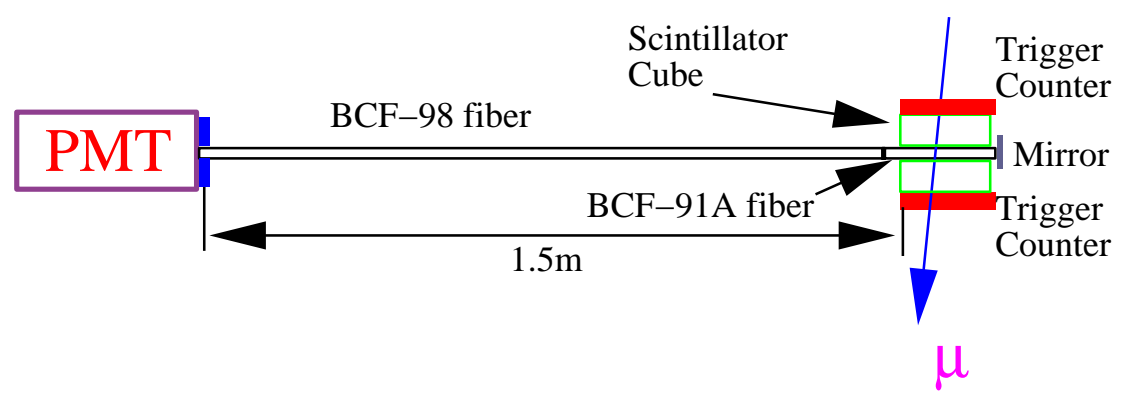

Figure 5.3: Setup used to test a prototype cube with multiclad fiber.

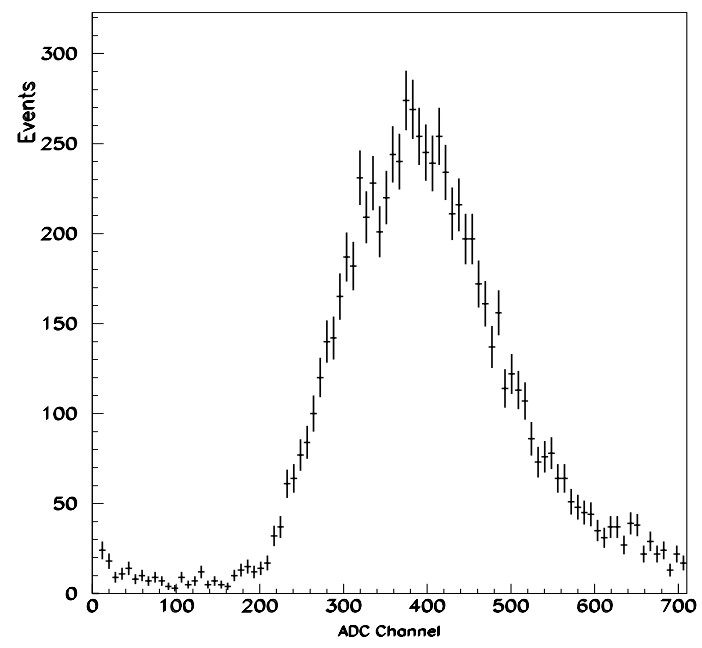

Figure 5.4: Pulse height spectrum of through-going cosmic ray muons obtained with a prototype cube with a $1.5 \mathrm{~m}$ multiclad fiber. 
which will go to a standard QT electronics board input. The other signal will go to a discriminator. The discriminator output can be used with the muon tracker signals for special triggers.

Stainless steel wire will hold the cubes in place. The installation of the final detector PMTs and support structure below the access portal will have to be designed to accommodate the installation of the cubes with their fibers and support wires. The cubes will be installed before the last detector section below the access portal is installed.

The cubes with the fibers and wires have a negligible impact on the performance of the rest of the detector. The cubes and fibers occupy only $10^{-6}$ of the volume and block less than $10^{-3}$ of the light created by events in the detector.

In the next 6 months, a cube will be tested in oil. The issue of enclosing the cubes and the fibers is being considered.

\subsection{Laser Calibration System}

A laser calibration system similar to the one successfully employed by LSND will be used. The primary purpose of this system is to quantify and monitor pertinent properties of each individual PMT including PMT gain, pulse height vs. photoelectron linearity, and timing. Other system functions include the monitoring of light attenuation of the oil over the lifetime of the experiment and the reconstruction of the light source location. The system will consist of a laser light distribution system, light dispersion flasks fixed at various locations in the detector, and long fiber optic cables which carry the laser light from the distribution system to each flask. Each $10 \mathrm{~cm}$ diameter flask will be filled with Ludox, a dispersive medium. We will use 200 micron quartz optical fibers.

The laser light distribution system will be housed in the utilities area. It will consist of a laser generating $\leq 5 \mathrm{~ns}$ pulses, a motor-controlled attenuation system, reference photodiodes and a mechanical splitter with a motor-driven movable mirror which would direct the laser light to individual optical fibers and a remote-controlled shutter blocking system. The Nitrogen/Dye laser will be similar to that used in LSND.

A stand-alone control system will operate the laser system running a realtime control program, such as LabView, on a dedicated PC. The reference photodiodes will provide a tag signal for the MiniBooNE data acquisition. The reference photosensors will be calibrated with low activity radioactive sources. As was done in the LSND experiment, the laser can be pulsed at $\sim 0.1 \mathrm{~Hz}$ continuously and asynchronously with the accelerator during normal data taking and at $20-30 \mathrm{~Hz}$ during beam off periods for special runs. Gain calibrations can be obtained from low light intensity runs by fitting the resolved single photoelectron peak for each individual PMT. The special runs can be with high light levels to provide timing offsets for all channels. Timing information from all the channels will be used to calculate a mean time slewing correction to be 


\begin{tabular}{|c|r|r|r|r|}
\hline radius $(\mathrm{m})$ & $\phi$ & $x$ & $y$ & $z$ \\
\hline 0 & $0^{\circ}$ & 0.00 & 0 & 0.0 \\
2 & $60^{\circ}$ & 1.73 & 0 & 1.0 \\
1 & $180^{\circ}$ & 0.00 & 1 & -1.0 \\
1 & $300^{\circ}$ & -0.87 & -1 & 0.5 \\
\hline
\end{tabular}

Table 5.2: Laser bulb positions are listed in this table. $x=r \sin (\phi)$ and $z=$ $r \cos (\phi)$

applied to each PMT.

Four flasks will be used. The first one will be located at the center of the tank. The next one will be placed in the $y=0$ plane $2 \mathrm{~m}$ away from the center at $\phi=60^{\circ}$. The third will be placed $1 \mathrm{~m}$ away from the center and $1 \mathrm{~m}$ above the $y=0$ plane at $\phi=180^{\circ}$. The fourth will be placed $1 \mathrm{~m}$ away from the center and $1 \mathrm{~m}$ below the $y=0$ plane at $\phi=300^{\circ}$. The laser flask positions are listed in Table 5.2.

The installation will depend on the same factors as the installation for the cubes. Consideration will be given so the two systems do not become entangled. Figure 5.5 shows the laser flasks, muon tracker, and scintillation cubes with the MiniBooNE detector. 


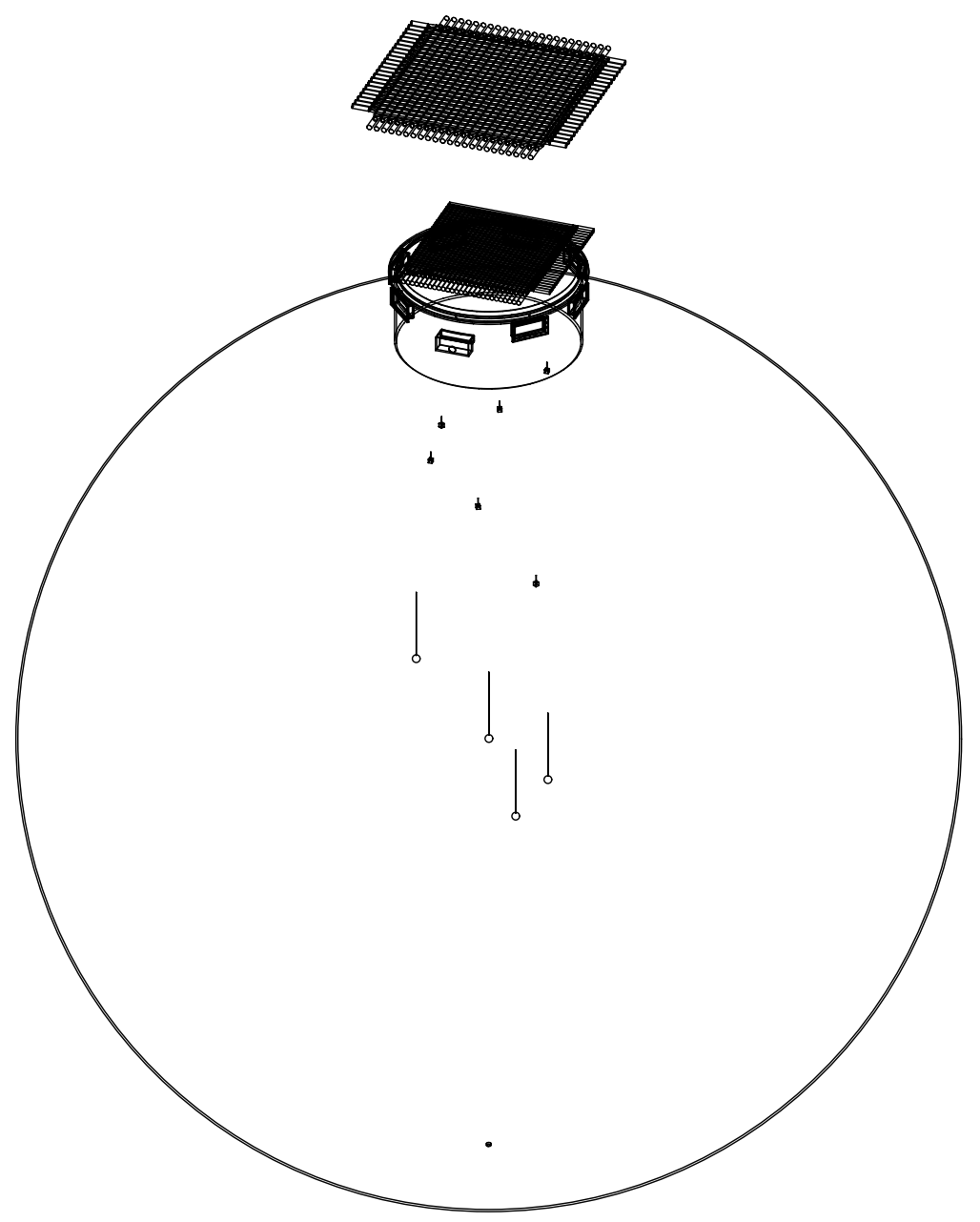

Figure 5.5: MiniBooNE Calibration Systems. 


\section{Chapter 6}

\section{Phototubes, Electronics, and Data Acquisition}

\subsection{Introduction}

In this chapter we describe the photomultiplier tubes, front-end electronics, data acquisition system, and electrical layout in detail. We start with the history and selection of the photomultipliers to be used in MiniBooNE, describe the testing, and go on to describe the high voltage and preamplifer systems. Details are given in Appendix 6A. The "QT electronics" is then described from a timeseries data collection point of view and is elaborated in detail in Appendix 6B. Command summaries and data formats used in the VME cards from which the system is built are given in Appendix 6C. The data acquisition and event assembly are discussed next. Upgrade plans are then given. The plans for beam and control signal monitoring are described. Details of a cable delay measuring scheme that integrates timing signals with the DAQ are given in Appendix 6D. The DAQ software is then discussed. Finally, the physical plant is described with emphasis on the cable routing and signal paths. The section ends with the four Appendices.

\subsection{Phototube Description}

This section provides a discussion of the phototmultiplier tubes, high voltage system, and preamplifiers for the MiniBooNE detector. The system had been in reliable operation in the LSND experiment from the fall of 1993 until 1998. It was taken apart and is in the process of being moved to Fermilab. The photomultiplier tubes were shipped to Fermilab in 1999 and were tested and recalibrated in the summer and fall of 2000. The 1220 LSND photomultiplier 
tubes (R1408) have been augmented by 300 new Hamamatsu R5912. They are being installed in the MiniBooNE detector tank this spring (2001).

The detector contains 1280 photomultiplier tubes that view the main volume of mineral oil and approxiamtely 250 photomultiplier tubes that view an annular volume located at the edge of the tank that constitutes a cosmic ray veto shield. The tubes are operated with a voltage gain of $8 \times 10^{6}$ and followed by a preamplifier providing a gain of 19 . The preamplifier system also provides the high voltage for the tube. A detailed description of the preamplifier electronics and the anode circuitry is provided in Appendix 6A.

The photomultiplier tubes to be used in MiniBooNE were developed in collaboration with the SNO experiment by Hamamatsu Photonics K.K. [7] The tubes LSND received were the first produced by Hamamatsu's production line, with SNO receiving the later tubes. The R1408 design is nearly ten years old and by present standards it is somewhat dated. The Hamamatsu R5912, which is a candidate tube for the full BooNE detector, is the heir to the R1408.

The LSND tubes (part number R1408 (modified)) are 8 inch diameter, 9stage hemispherical photocathode photomultiplier tubes fabricated out of Schott 8246 low radioactivity glass. A photograph of the R1408 in its wire frame mount is shown in Fig. 6.1. Because of the need for good timing, the Hamamatsu designers elected to use a 9 stage design and apportion half the voltage across the tube to the photocathode-first-dynode potential. This reduces the transit time spread of single photoelectron events. It also raises the problem of dark pulses arising from field emission from electrode elements inside the tube. Due to production schedules, the design was frozen. The production tubes could operate at a gain of only about $8 \times 10^{6}$ before the dark pulse rate would overwhelm the anode signal. At this gain the dark pulse rate at a threshold of one quarter of a photoelectron was 2000 - 7000 counts per second. Operation at the lower gain necessitated the addition of preamplifiers to boost the $1.5 \mathrm{mV}$ anode pulses corresponding to single photoelectron events. The time resolution was good at approximately $1.9 \mathrm{~ns}$ rms and was reasonably uniform across the sample of 1220 units that LSND received. Single photoelectron charge resolution had a peak-to-valley ratio of approximately 1.1, which was less than desirable. An example of the test results from our early testing is shown in Fig. 6.2. A problem with the tubes was their anode charge spectrum for single photoelectron levels of illumination. They exhibit a rather long tail indicative of nonuniform first dynode surface sensitivity. This problem may be characteristic of the focusing system and mesh dynode structure used by this tube. For the experiment this means that a probability weighting must be assigned to any large pulse and processed accordingly. For very large light pulses, such as from through-going cosmic rays, the tubes exhibit afterpulsing at delays of $5 \mu \mathrm{s}$. This probably arose from residual gas inside the tube envelope and was handled by the data acquisition electronics. The tube was relatively inexpensive and provided a very cost effective way of buying photocathode area per dollar.

As it was a special production run, the R1408 as modified is no longer manu- 


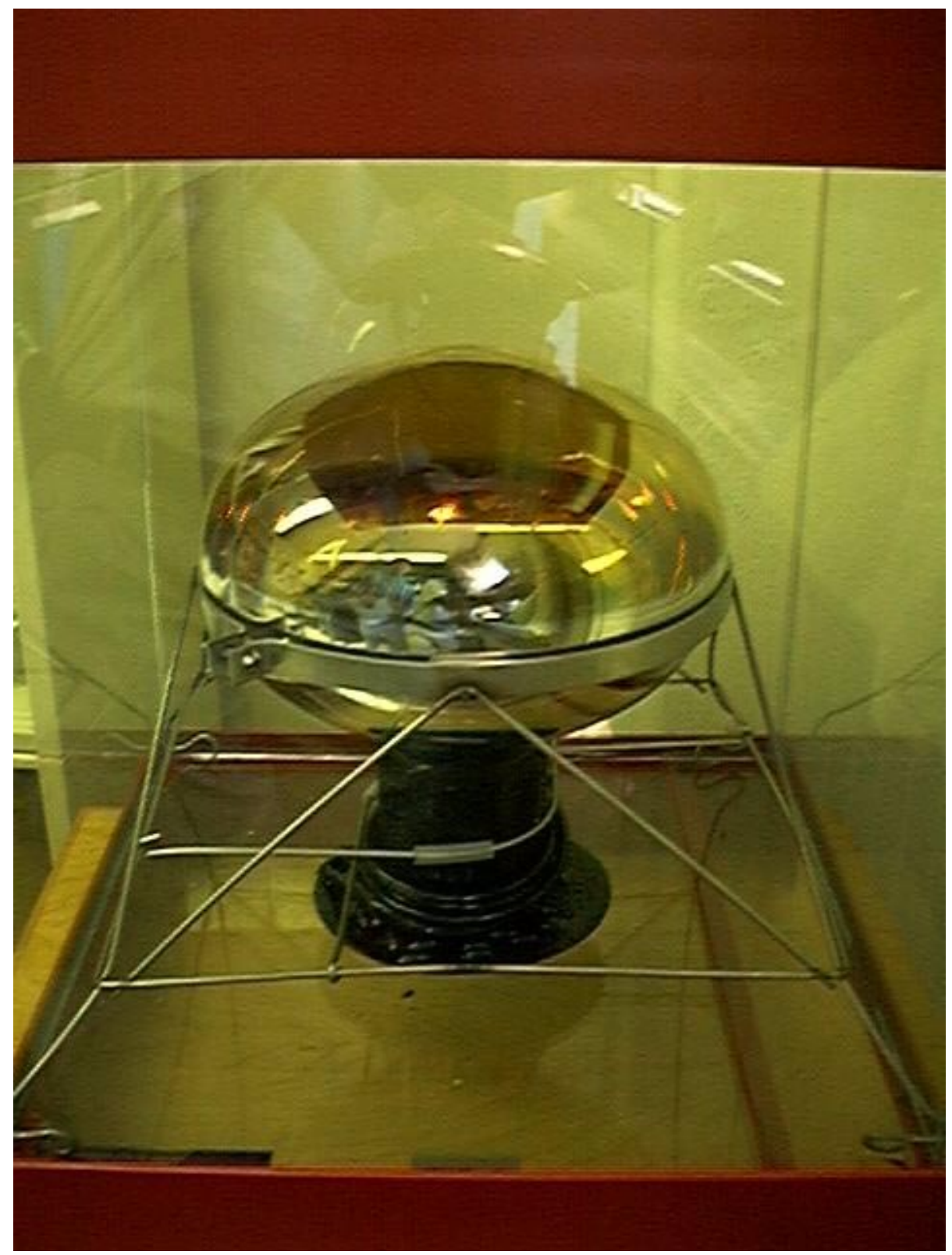

Figure 6.1: Photograph of the Hamamatsu R1408(modified) 8 inch diameter, 9-dynode stage photomultiplier tube to be used in the MiniBooNE detector. It is shown in its wire-frame mounting fixture. 


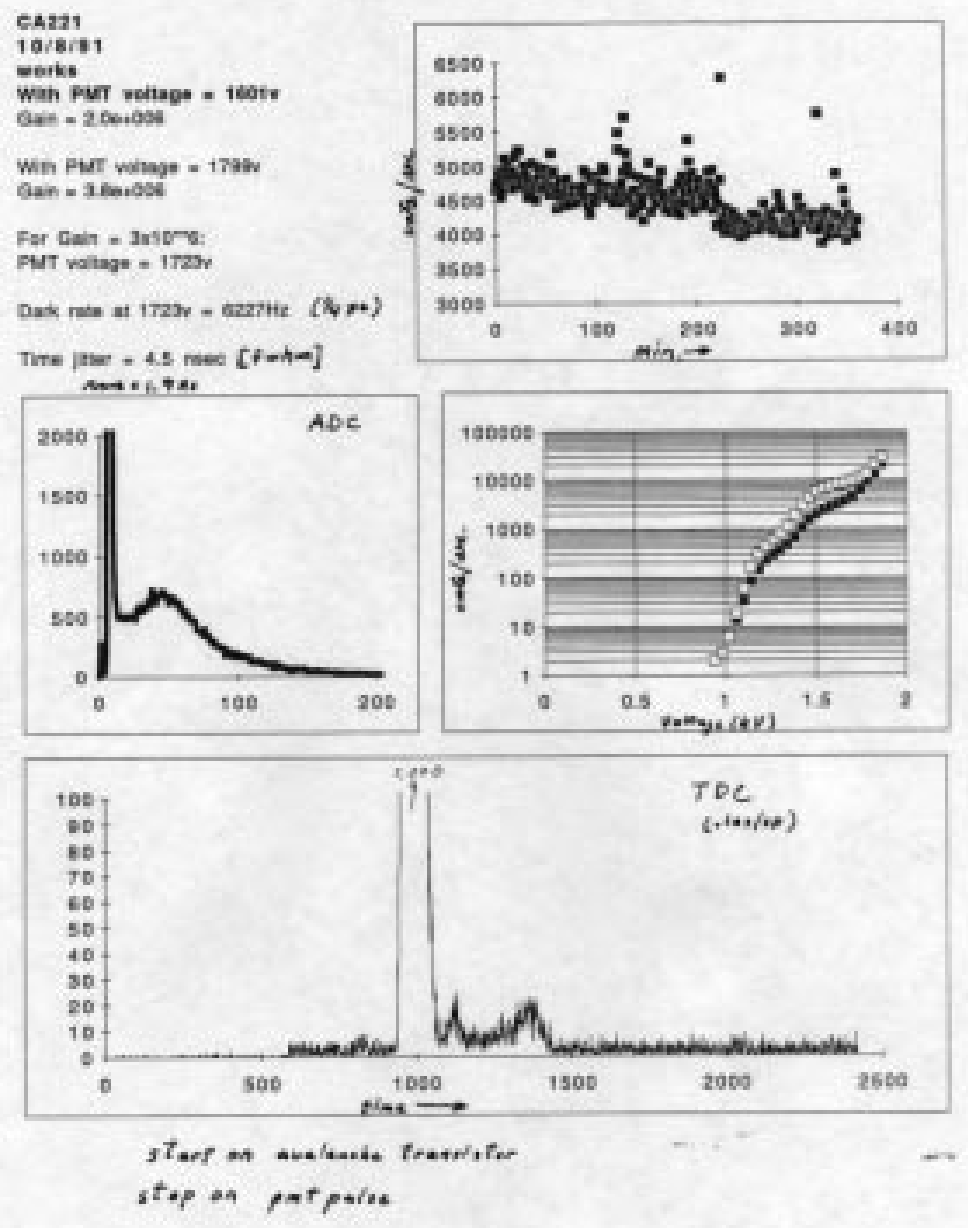

Figure 6.2: An example of a summary of test results of a typical R1408 photomultiplier. 
factured; however, Hamamatsu has continued the development of 8 inch diameter tubes and has brought to market a mature and stable tube, the R5912. The R5912 is a 10 stage design (producing clean $25 \mathrm{mV}$ anode pulses corresponding to single photoelectron events at a realistic operating voltage) that has reasonable charge resolution, good timing, and a low dark rate.[8] It is a production tube that is available from Hamamatsu. The detailed properties, such as charge spectrum for single photoelectron events, prepulsing and afterpulsing, and time and charge resolution, were evaluated in a 300 tube sample and found to compare very well with the existing R1408. A final report on the test results is being written.

Bases for both the R1408 and the R5912 have been designed, tested, and fabricated. The R1408 base is shown in Fig. 6.3 and a test base for a ten-stage version of the R5912 is shown in Fig. 6.4. The base voltage tapers are based on those recommended by Hamamatsu and are primarily linear gradients. Approximately one half of the total applied voltage appears across the cathode-first dynode gap to minimize electron transit-time spread (which is inversely proportional to this voltage) and to insure good collection efficiency. The anode circuit has been designed to realize good fast-pulse performance, clean baseline recovery, and $50 \mathrm{ohm}$ back termination. The tubes operate with the anode at positive high voltage and the cathode at ground, as is necessary for proper operation of these tubes. We adopted a single-cable design to simplify the cable plant and to minimize the inert material in the detector. The resulting capacitive decoupling introduces approximately $1 \mu \mathrm{s}$ of ballistic overshoot, but, as the front-end electronics integrates and digitizes the integral in $100 \mathrm{~ns}$ intervals, this is not a problem. A SPICE simulation of the anode end of the dynode chain is shown in Fig. 6.5 and a recorded pulse in the real system is shown in Fig. 6.7.

The bases were attached at the time of acceptance testing. The tubes have glass pinouts and the printed circuit base is soldered directly to the leads. This method of attachment was preferable due to the required reliability of the connections and the low shear stress it applied to the glass-metal seals. We have encountered no problems with the short term thermal stresses produced when soldering. Tubes that passed as acceptable then had their bases coated in three layers of a coating material to seal their components and protect the base from the mineral oil and visa versa.

The R1408 PMTs only had 9 dynode stages and produced low outputs of $1.5 \mathrm{mV}$ for single photoelectron signals. To raise this and improve our signal to noise, a stage of $\mathrm{x} 19$ preamplification was added to the high voltage distribution system. The circuit is shown in Fig. 6.6. Careful attention was paid to the grounding and power returns of these amplifiers, such that when connected to the high voltage and to their appropriate QT channels (see below) there were effectively no ground loops. Tube gain was set by the series trim resistors on the inputs to the preamps. This makes a simple high voltage system that is stable and allows the use of fixed voltage supplies to power all the tubes. A typical anode pulse and its integral are shown in Fig. 6.7 


\section{Hamamatsu R1408 8" Diameter 9 Dynode Photomultiplier Tube}

Used in the Large Scintillator Neutrino Detector (LSND)

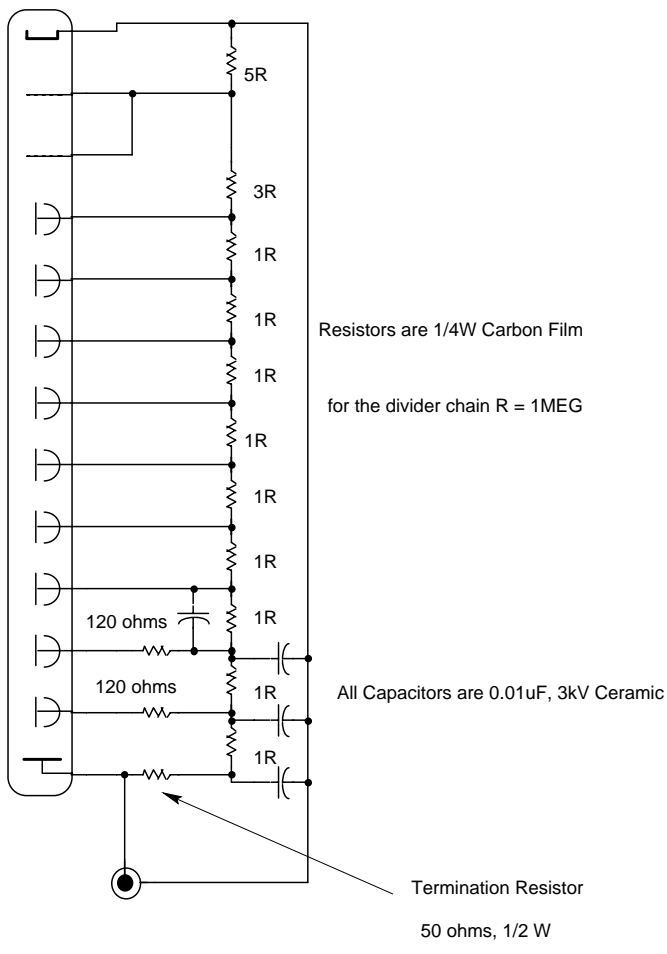

Figure 6.3: Schematic diagram of the base used for the R1408 photomultiplier tube. 


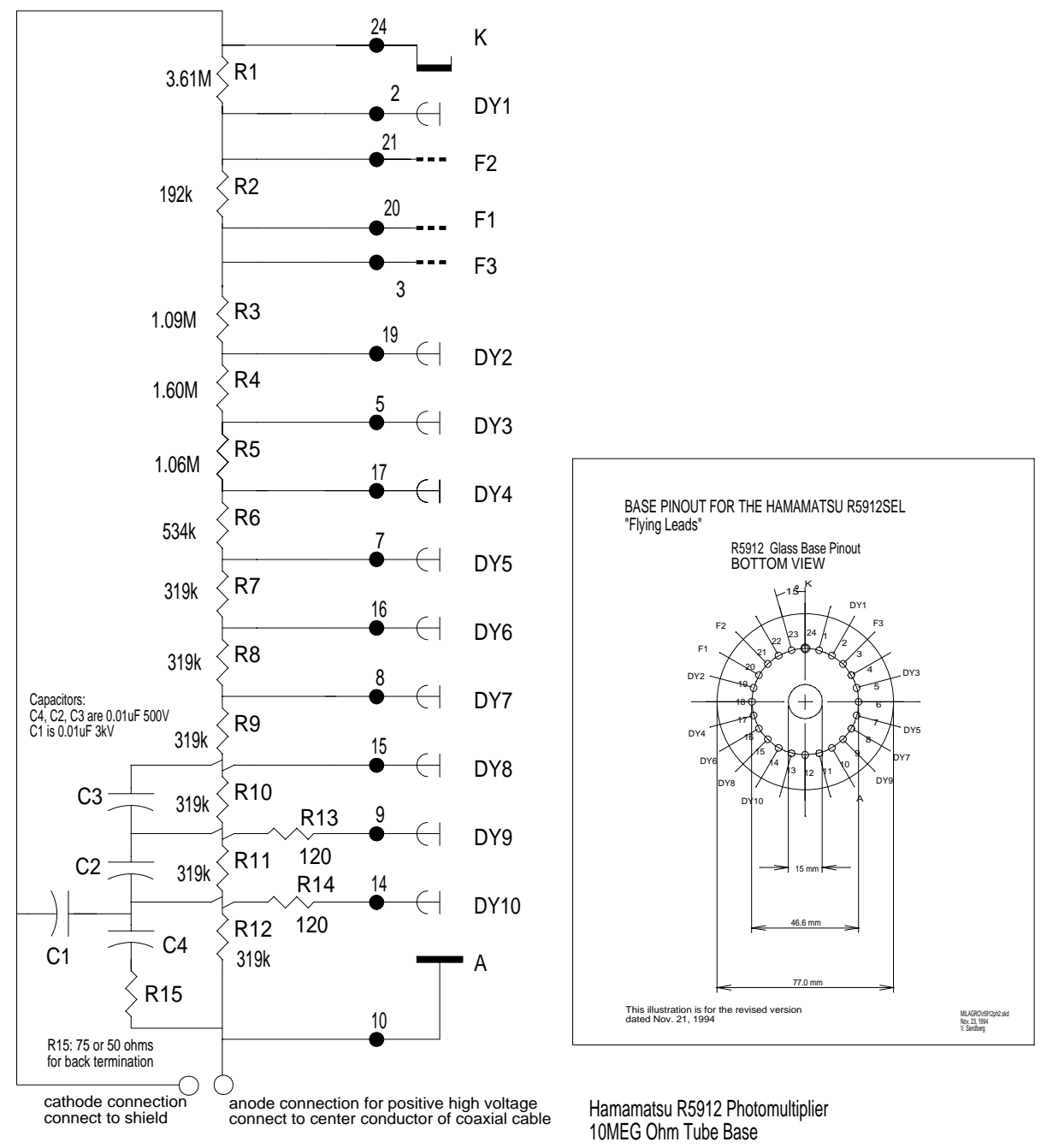

Figure 6.4: Schematic diagram of the base used for a 10 dynode stage version of the R5912 photomultiplier tube. 


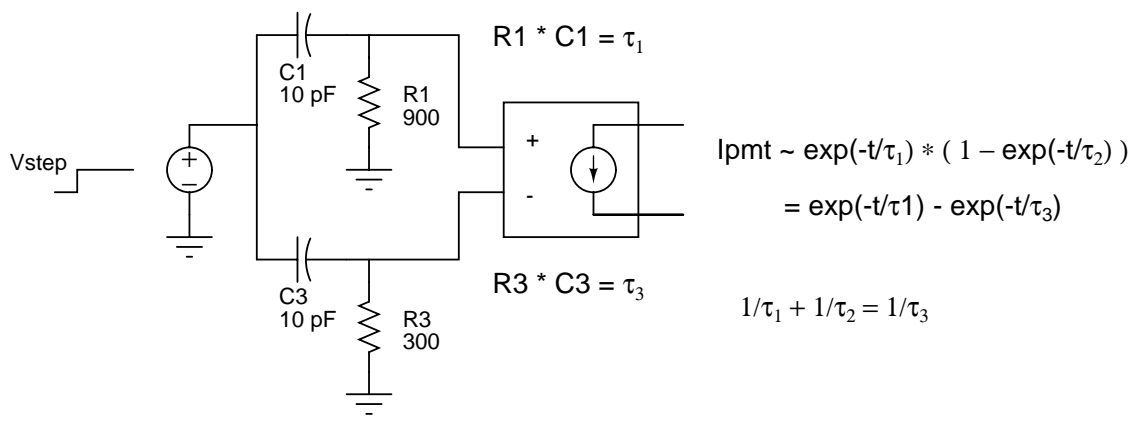

PMT Anode Wavform SPICE Model

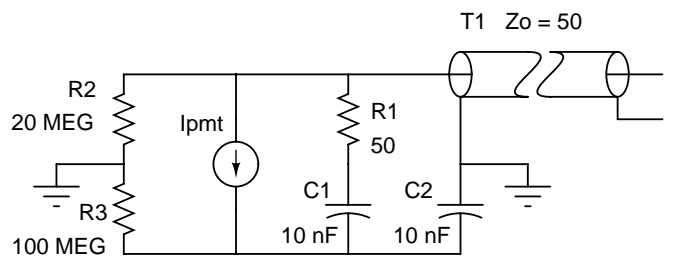

SPICE Model of PMT Cable Driving Circuit

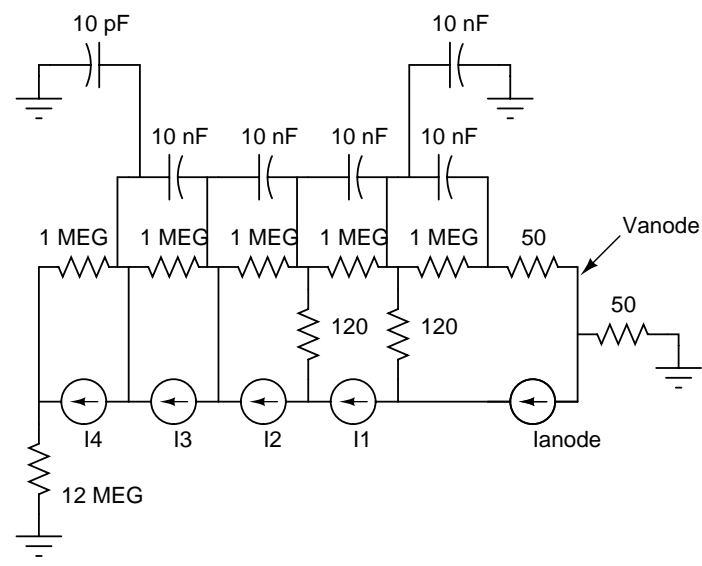

SPICE Model of PMT Dynode Chain and Anode

Figure 6.5: SPICE model of the photomultiplier tube dynode chain and anode circuitry. 


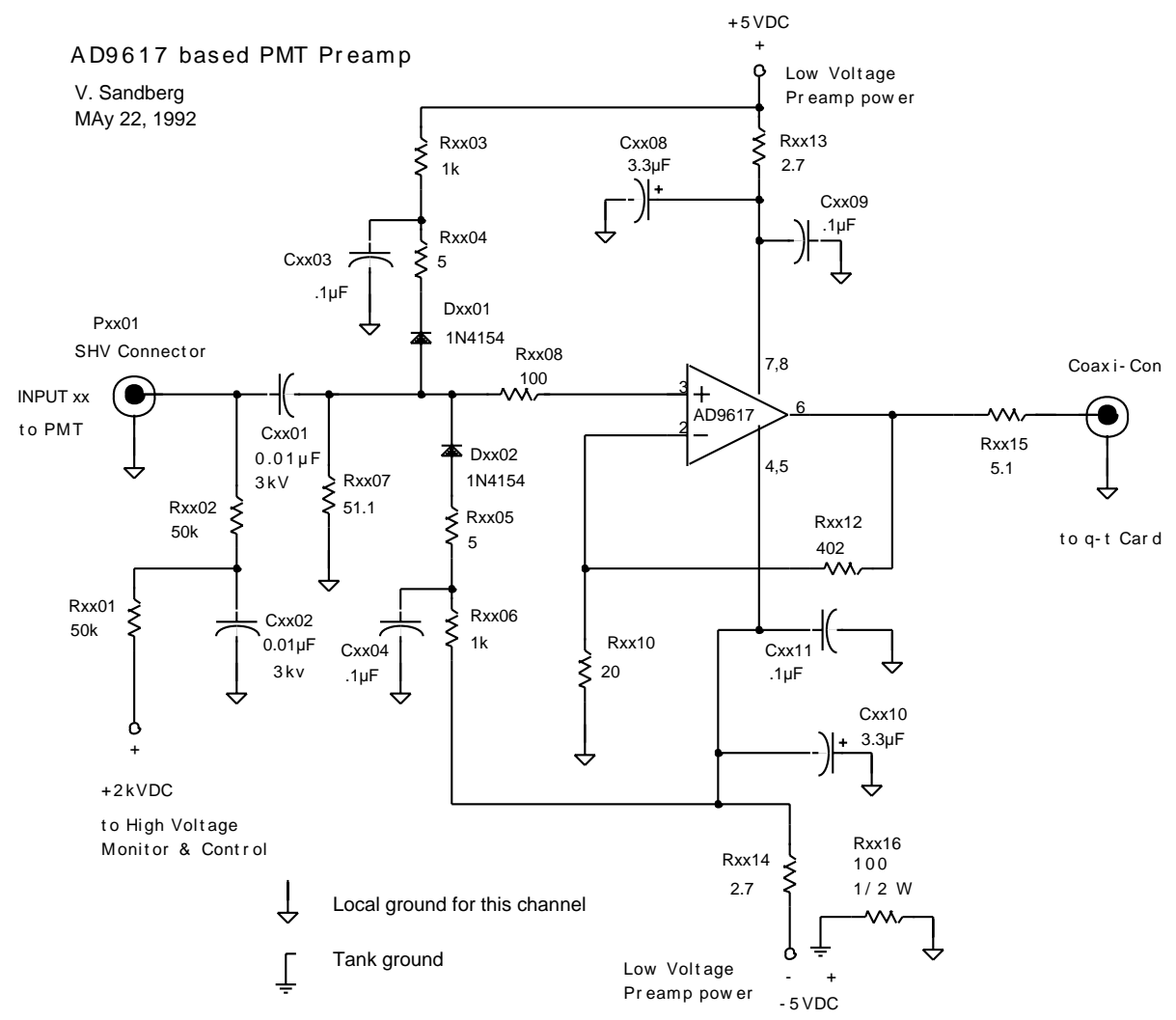

Figure 6.6: The preamplifer and high voltage circuit used with the photomultiplier tubes. 


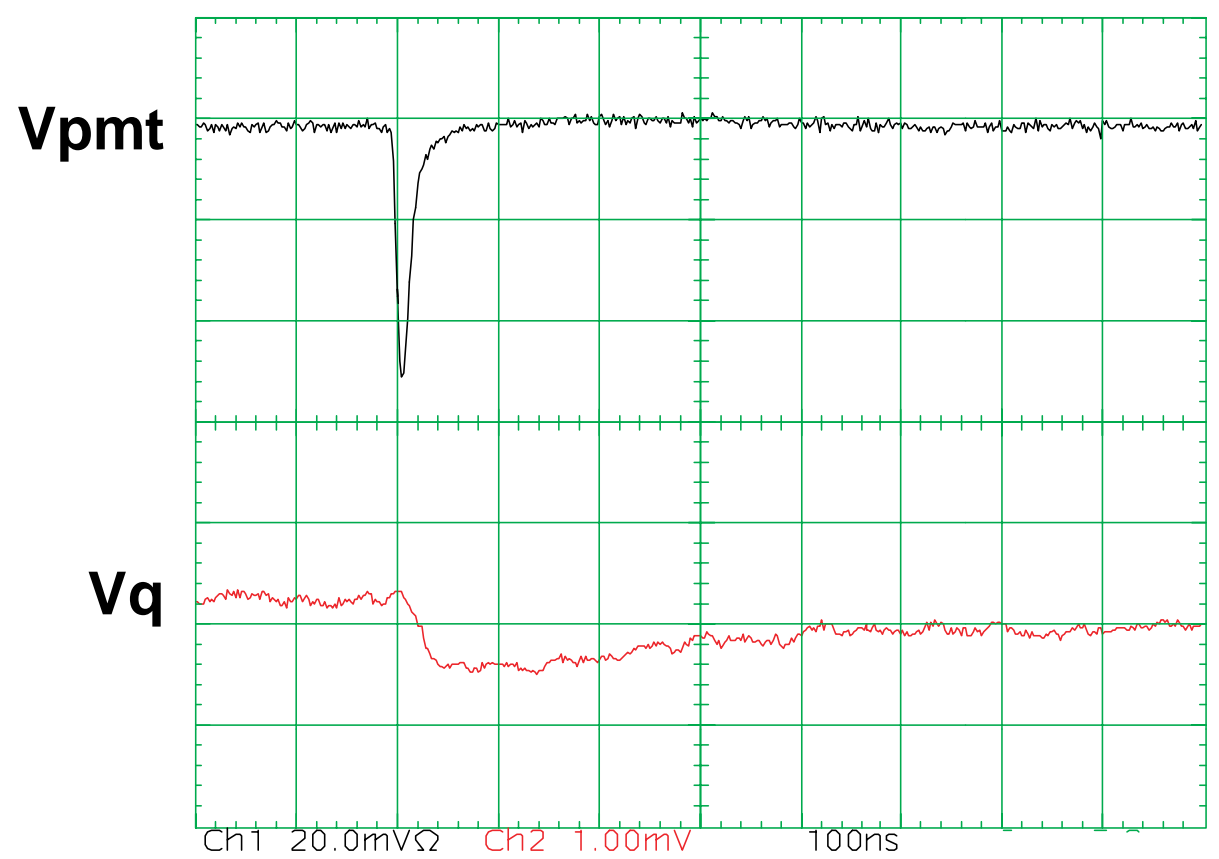

Figure 6.7: Typical waveform of an anode pulse and its integral. 


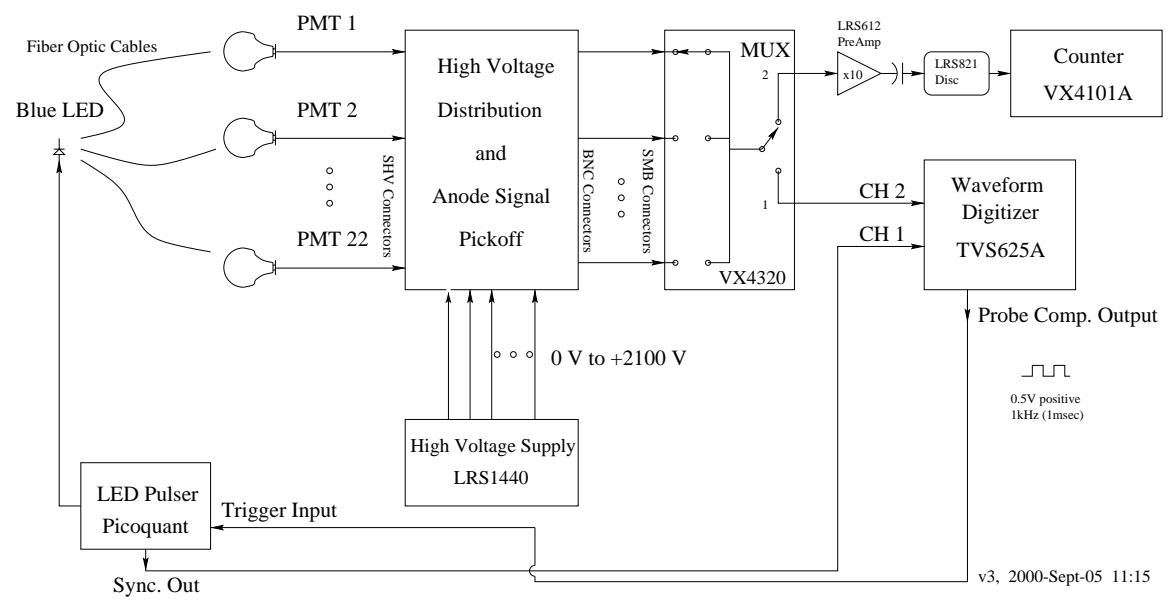

Figure 6.8: Photomultiplier tube teststand schematic.

\subsection{Phototube Testing}

A test program to determine the characteristics was carried out during the summer and fall of 2000. The program evaluated the characteristics of the photomultiplier tube for light pulses of duration and spectral content expected from the MiniBooNE Cerenkov signals. The system employed a pulsed blue LED system with $200 \mathrm{ps}$ rise and fall times and full anode waveform capture with a fast $(5 \mathrm{GSa} / \mathrm{s})$ digitizing system. A schematic of the pmt teststand is shown in Fig. 6.8

Approximately 1000 waveforms were acquired for each of the photomultiplier tubes at the single photoelectron level. The waveforms were stored and archived. By acquiring and recording the time history of the tube under test with subnanosecond resolution, many aspects of the tube's performance can be extracted and analyzed using techniques more familiar to the contemporary class of physicists (who are more at home with a computer than with hardware). For example, the time and charge resolutions and their correlation may be extracted by simulating the analysis done with a discriminator and a gated ADC in software. Each recorded waveform is processed as a time series and the histograms of interest are generated. In particular, the difficult determination of the prepulse and postpulse probabilities are relatively simple to extract. The problematic large postpulse distribution can be determined. The method produced an archive of waveforms that will be used to design signal processing hardware matched to the tube for the full BooNE detector. The system will archives a given tube's performance and will be of great use for later detailed analysis of the experiment's data. 


\subsection{Electronics Description}

The front-end electronics and data acquisition system built for LSND and to be used in MiniBooNE was designed to acquire charge and timing information from photomultiplier anode signals and to store them in a way that allowed for efficient sorting and event building. The limit then as now was the available computing power for a limited budget. The design is a compromise between collecting enough data at the front end to make the required measurement and the limits on computational capability and data transmission limits of communication networks. A front end of transient digitizers can collect the full time development of a pulse, but for a system of 1200 or more channels the amount of data that needs to be transfered from the digitizer memory to the main computer's memory and the subsequent processing presents an enormous burden on system resources. Our approach takes a different route.

For MiniBooNE, front-end fast-analog circuits process the anode pulses, which have significant frequency components up to several hundred $\mathrm{MHz}$, to create "slower" analog voltages that are proportional to the charge $(\mathrm{Vq})$ in the pulse and to the time the pulse occurs with respect to a global system clock (Vt). This is illustrated in Fig. 6.9. The Vq and Vt signals are digitized by two flash ADCs operating at $10 \mathrm{MHz}$ and stored in circular buffer memories. A global, GPS referenced $10 \mathrm{MHz}$ clock provides the timing signal for the ADCs, time reference signals, and FADC memory address generation. The charge and time information of an anode pulse are stored in the buffer at an address corresponding to counted $100 \mathrm{~ns}$ clock ticks from the $10 \mathrm{MHz}$ clock. This system is illustrated in Fig. 6.10. Each photomultiplier tube is connected to such a system. Upon receipt of a trigger, a "trigger broadcast module" sends out a set of "time stamp addresses" to the buffer memories. Data is transfered from the buffer memories to a set of FIFOs that hold the data until it can be read by a single board VME-based computer (SBC). The system is packaged so that 128 PMT channels are handled by one SBC. As explained in more detail below, these computers process the charge and time data in the different time windows and reduce the time series to simple charge values and the time they occured to subnanosecond resolution for each tube. The reduced data is then sent over serial cables (both fiber optic and copper coaxial) using ethernet protocol to the main acquisition computer. There the entire data set for all the tubes in the detector is assembled and recorded to archive media.

Each photomultiplier tube signal is connected to a channel that performs a leading edge time interval determination (the interval being between the PMT pulse and the following edge of the global clock) by starting a linear ramp on the discriminator edge and resetting the ramp on the second subsequent clock tick, and an integration of the pulses to determine its charge. These circuits are described in more detail in Appendix 6B. The charge and time signals are sampled every 100ns by FADCs and the digitized data are written into dual ported memories addressed by the global time base. The acquisition 


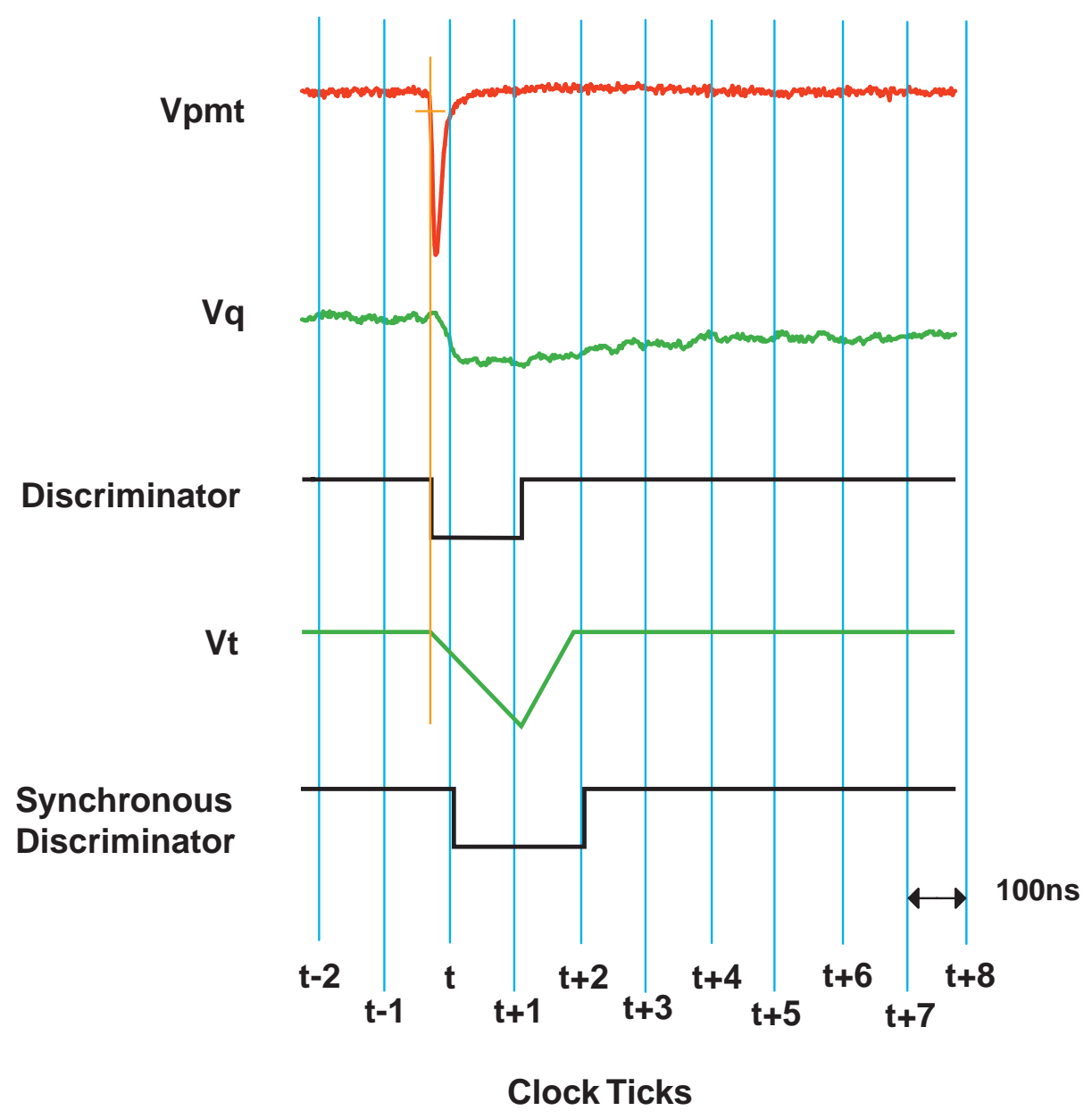

Figure 6.9: Charge and time data capture for a PMT pulse. The upper trace is the anode pulse and the second trace is the integrated charge. The "Discriminator" trace is a digital signal created when the anode pulse crosses a preset threshold. The "Vt" trace is the analog time ramp that starts with the "Discriminator" signal. The "Synchronous Discriminator" trace represents a signal that may be used by the trigger electronics. The Vq and Vt are analog signals that are digitized by FADCs at the sample times shown by the vertical lines, labeled by the clock ticks. 


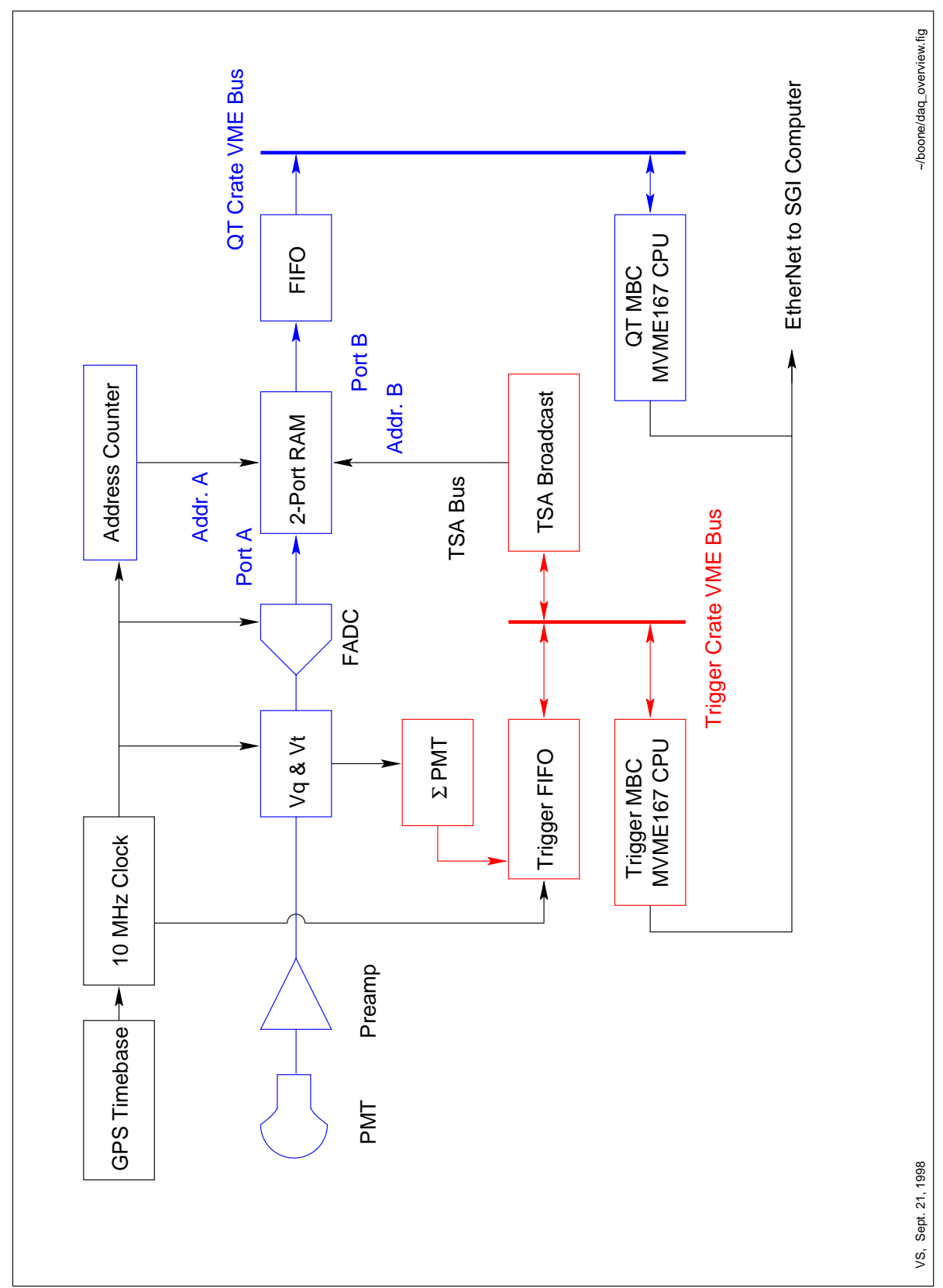

Figure 6.10: Illustration of the MiniBooNE data acquisition system, showing the FADCs, circular buffer, and event building data streams. 


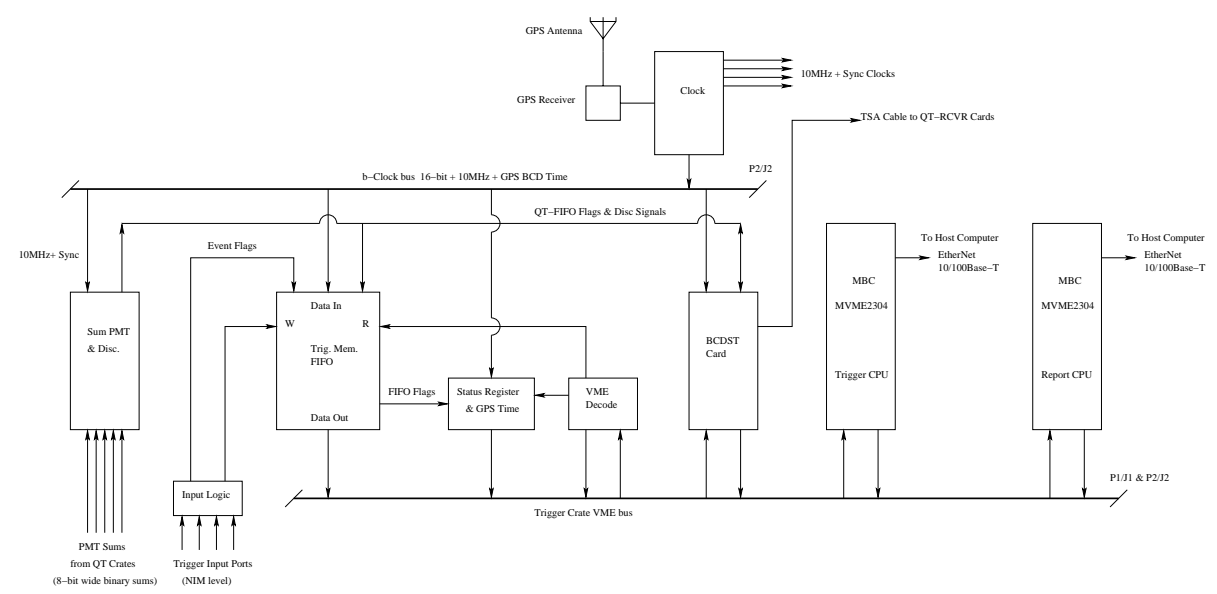

Figure 6.11: The trigger hardware layout.

of a PMT pulse, its integral (Vq), and the time-interpolating ramp (Vt) are illustrated in Fig. 6.9. At each clock tick the charge and time analog voltages are digitized by the FADCs and written into the dual ported memory (the QT memories). The address is then incremented and the cycle repeated. This part of the acquisition system is free running. The memories are necessarily finite in size and hold a finite time history (for LSND 204.8us with 100ns resolution). They are configured as circular buffers, that is, the memory is overwritten after the clock has cycled through the address space of the memory. This provides for temporary storage of the immediate past history for a time long enough for a trigger decision to be made. It also allows for a pretrigger to determine the state of the detector prior to the arrival of beam.

Data are extracted from the front-end dual-ported memories and built into events by the trigger system. The trigger computer initiates event building by broadcasting those time addresses when the particular beam extraction took place to the second address port of the dual-ported memories used in the circular buffers. The trigger crate layout is illustrated in Fig. 6.11. The trigger logic is illustrated in Fig. 6.12. The system transfers the data at these "time stamps" from their temporary location to FIFOs located adjacent to the dual-ported memories, where the data resides until read by other single board computers located in each VME crate. These computers (MVME-167) calibrate and compact the data, assemble them into ethernet packets, and send them to the experiment's host computer.

An extra PMT channel is used to record the Booster extraction kicker signal 


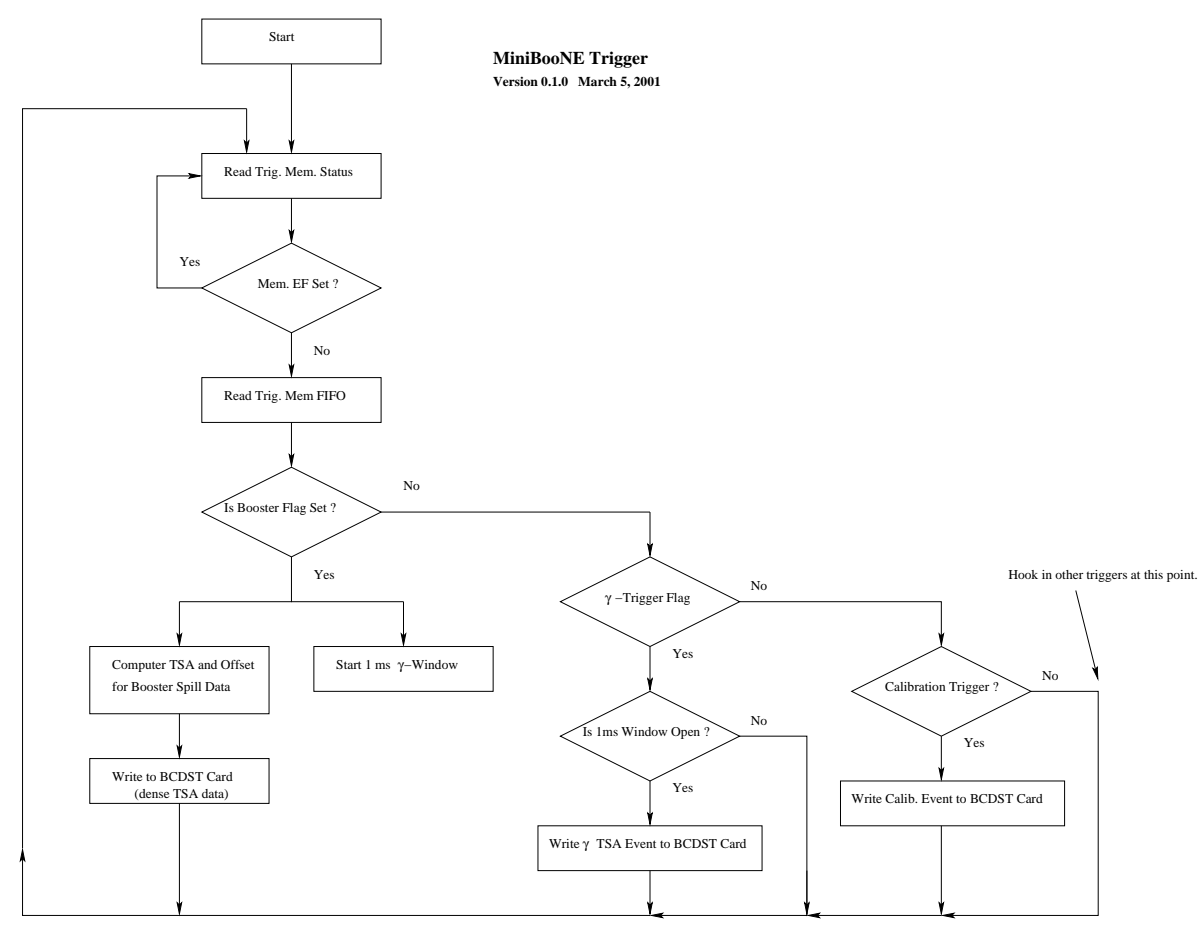

Figure 6.12: The trigger logic. 
and synchronize its timing with the events in the MiniBooNE detector. This is discussed in more detail below and in Appendix 6D.

This system is built in modular form and consists of a single trigger-clock crate and multiple "QT crates". Each QT crate is an autonomous pulse acquisition system capable of recording up to 128 independent channels. A QT crate consists of a SBC, a "receiver card" (RCVR), up to 16 8-channel "QT cards", and a "summation card". The RCVR card controls communication between the QT crate and the trigger crate. The summation card computes an 8-bit binary sum of the number of PMT channels that were "on" during the previous $100 \mathrm{~ns}$ interval. The sum signal was used in LSND to generate a multiplicity trigger for event selection. For MiniBooNE, as mentioned above, a different trigger design, based on the Booster extraction kicker, will be used.

The trigger crate contains two SBCs, a "Broadcast card", the $10 \mathrm{MHz}$ clock, "memory card", and trigger-input card. One of the SBCs is the master of the whole experiment. It controls and coordinates all signals in the trigger VME crate. The clock card receives data from a GPS clock, which includes date and time accurate to $1 \mu$ s and the $10 \mathrm{MHz}$ time base, generates a "binary clock" used to address the circular buffer memories and to provide the coarse $100 \mathrm{~ns}$ timing, and distributes buffered copies of these signals to the QT crates and to a dedicated backplane in the trigger crate. (Actually, to simplify things only the $10 \mathrm{MHz}$ signal and a synchronizing signal. The RCVR cards receive this pair of "clock" signals and generate their own binary clock for addressing their QT cards' memories.) The trigger-input card accepts signals from outside, such as the Booster extraction kicker signal or the protons on target signal from the horn, and uses it to load a binary clock time (from the trigger crate backplane) onto a FIFO stack. This FIFO is read by the first SBC and uses this number to determine the starting address to send to the broadcast card. The broadcast card uses this starting address, minus an offset to allow for delay in the creation and propagation of the trigger signals and to collect a pre-beam sample of the detector history, and broadcasts this address and subsequent address increments to the RCVR cards over the "TSA" cable. The TSA cable provides a connection between the trigger broadcast card and the RCVR cards in each QT crate. The RCVR cards stack the TSAs on a "RCVR FIFO" and send them to the QT cards in the QT crates. In the QT cards the data at the prescribed address is transfered from the QT buffer memory to the QT FIFOs, where it resides in nonvolatile storage until read by the $\mathrm{SBC}$ in the crate.

The SBC in a QT crate detects the presence of data to be read by polling the RCVR FIFO empty flag, located as the msb in the status word generated by the RCVR card. When the presence of data is detected, the SBC begins a series of reads of the RCVR and QT cards. The address space of each QT crate has been arranged so that the data are located in contiguous locations. The SBC, a Motorola MVME167, can execute the contiguous reads efficiently, one $200 \mathrm{~ns}$ long VME cycle per 32-bit word. The data are read from RCVR and QT FIFOs into the SBC, where it is processed and then sent in ethernet packets 
to the host computer. (The abbreviations "MBC" and "SBC" are used as synonyms throughout this report. The "MBC" is commonly used by Motorola, where there seems to be an emphasis on the "M", whereas the more generic "SBC" for single board computer is used when the device in question is not so specific a Motorola product.)

The pulse acquisition system is composed of three independent loops: The front-end FADCs that sample the charge and time signals (which were derived from the PMT anode pulses by fast analog filters) and write their data into circular buffers (constructed from 2,048 deep dual-ported memories), the trigger system that receives signals from beam-related timing signals and controls the transfer of QT data from the buffers to the holding QT FIFOs, and the event building SBCs that read the RCVR and QT FIFOs, process and compress the data, and send it to the host computer through ethernet connections. The commands and data structures used in this system are summarized in Appendix 6C. The modules and their interconnections for a typical "QT crate" are illustrated in Fig. 6.13.

The system is packaged in $139 \mathrm{U}$ VME crates. The trigger electronics resides in a standard $6 \mathrm{U}$ VME crate and contains the global clock, the specialized trigger cards and the SBCs. Thirteen of the crates are called "QT Crates" and contain 16 "QT Cards" with 8 PMT channels per card, a time stamp receiver card that handles communication with the trigger, and a PMT Summation Card that produces the 8-bit crate sum every clock tick. Each QT Crate processes 128 PMT channels. The system can be extended indefinitely by adding additional QT crates. A layout of the 14 crate system is shown in Fig. 6.14. A common "TSA bus" runs from the trigger broadcast card to all the QT RCVR cards and is terminated at the last QT crate. Physically the TSA bus is a flat ribbon cable built up out of 32 channels of twisted pair cables. It conducts PECL-level logic signals that make up the time-stamp addresses and the TTL-level FIFO status flags. Separate, dedicated two-channel twisted pair cables run from the clock card to each of the QT RCVR cards. The clock cables are all the same length (to within approximately $0.5 \mathrm{~ns}$ ) and carry the $10 \mathrm{MHz}$ clock and clock synchronization PECL-level signals. Finally, cables link all the SBCs and the host computer via multiple ethernets.

In the analysis computer, sometimes referred to as the host computer, the full data for an event are assembled. At this point an event consists of the charge, coarse time (i.e., the timestamp value), the fine time, and the PMT number of each PMT that received a light pulse. In addition there are data that indicate the status of a calibration laser and the state of the beam.

\subsection{Electronics Upgrade}

The LSND photomultiplier tubes and the LSND electronics will be used for MiniBooNE. We plan on using new cables from the PMTs to the preamplifiers 


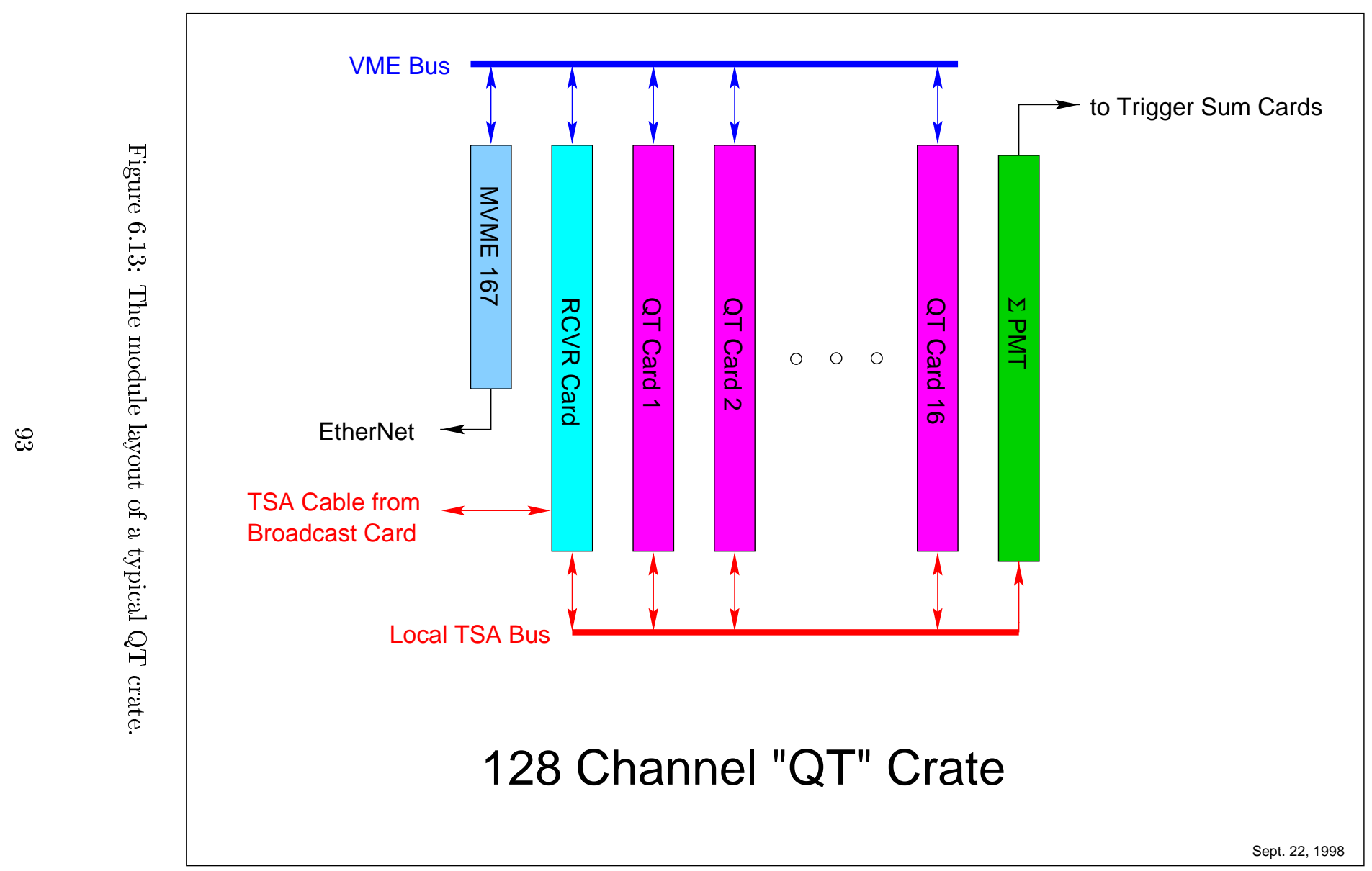




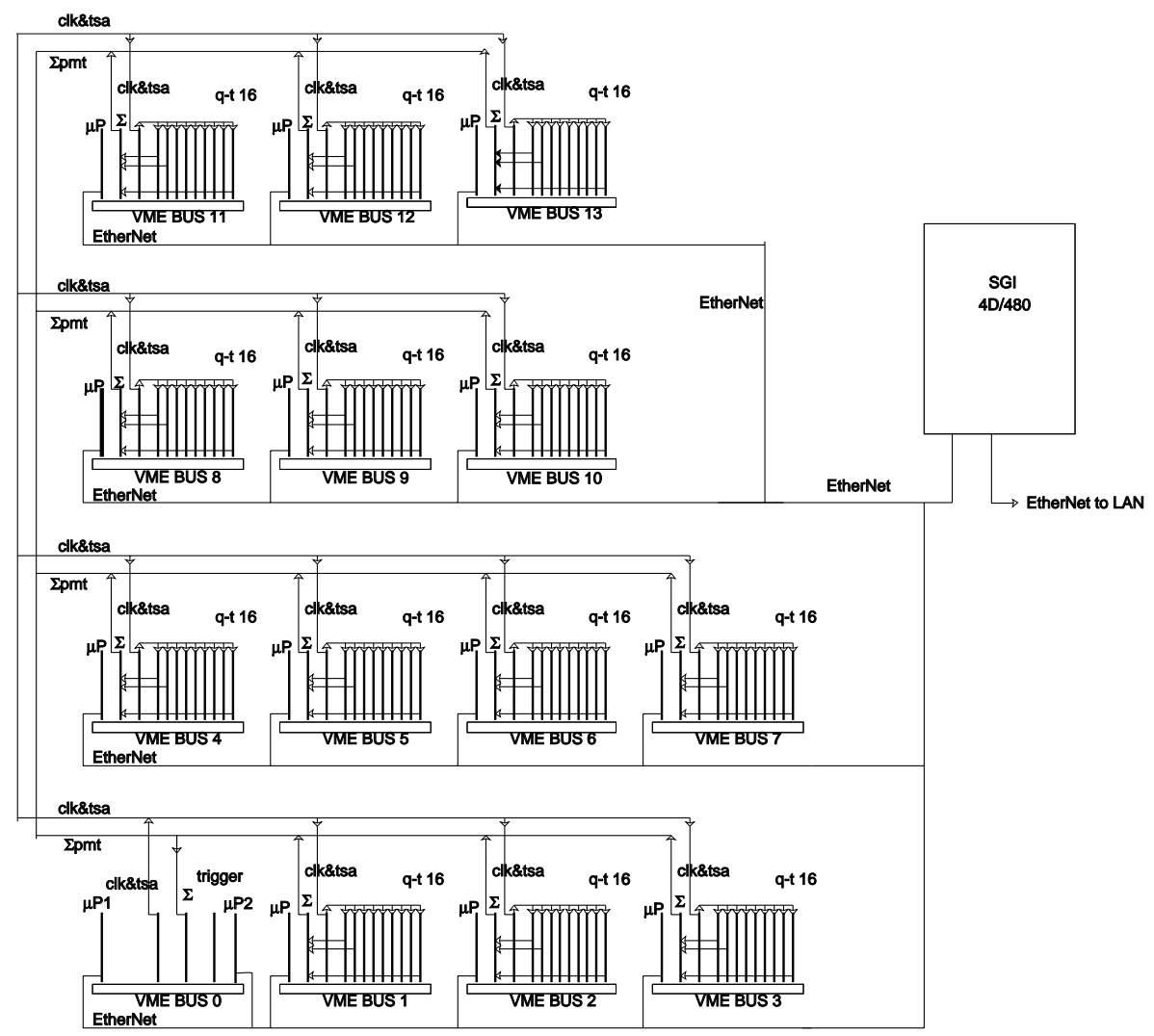

Figure 6.14: Layout of the MiniBooNE DAQ system. Shown are the arrangement of the $13 \mathrm{QT}$ data acquisition crates and the Trigger crate. 
and on buying new high voltage power supplies. (Typically six each, Bertan $310-01 \mathrm{~s} 0-3 \mathrm{kV}, 75 \mathrm{~mA}$ or equivalent units. The use of multiple units keeps the power density down and improves reliability.) The QT crates will have new cable assemblies and some connectors will be replaced. The trigger system will require that the Input card be designed and fabricated. This work is ongoing at present.

New code will be developed to handle the different types of event building we anticipate to use in MiniBooNE. LSND was multiplicity-trigger driven and built small time-window events of $500 \mathrm{~ns}$ width, whereas MiniBooNE will be triggered on the Booster extraction kicker signal, and a wide time window several tens of microseconds wide will be collected around this signal. We plan to redesign the initialization and control software that starts up and synchronizes the SBCs. The "real time Linux" system will be used for the DAQ.

\subsection{Beam Monitoring}

In order to correlate event data with beam data a system capable of recording beam timing data at the detector to nanosecond resolution will be necessary. To accomplish this there will be a straight-shot cable duct from the target service building to the detector which will be used to carry beam timing signals. In particular it will carry:

1. The Tevatron (accelerator) clock (for microsecond-level timing).

2. A "beam on target" signal (for nanosec-level timing).

The Tevatron clock with suitable electronics will be used to create a readout gate for the detector. The nominal $1.6 \mu$ s spill will be overlapped by a comfortable margin so that $\mu$ s-level timing is adequate. The "beam on target" signal will be used to determine when neutrinos from each RF bucket reach the detector and thus needs to be stable to the nanosecond level. A scheme is described in Appendix 6D can be used to calibrate (event by event) the cable delay and record the timing signal through dedicated QT-channels. The detector DAQ system records multiple data channels (approximately 1600 channels) simultaneously to subnanosecond resolution. This makes synchronizing the beam timing and detector-event timing relatively straightforward. The neutrino flight time can then be calibrated by using $\nu_{\mu} C \rightarrow N \mu$ events in the detector using data collected in month-by-month intervals.

Possible sources for the beam on target signal are:

1. Discriminating a beam line monitor (such as a BPM)

2. Extending the Booster Beam extraction signal to the detector. This signal comes out of the low level RF and tells the Booster extraction elements to begin extraction. Kickers, for example, begin a countdown of RF cycles 
to fire. (The countdown is around 17500 so that beam is extracted some $330 \mu \mathrm{s}$ after the signal). This signal is also encoded as a Tevatron clock event and is one of the events that would be used to derive a DAQ gate for the detector.

The exact design will depend upon the detailed properties of the available signals; but, as the above discussion illustrates, adequate information is available and the proposed system is capable of handling such signals.

Beam line information (magnet currents, BPM readings, SEED and multiwire profiles, etc.) will be collected and GPS timestamped in a separate beamline DAQ. This data will be sent to the detector DAQ computer via computer network messages and written to tape along with the detector data. The GPS timestamps will be used to synchronize the detector events with the beam information.

\subsection{DAQ Software}

This section discusses the software control of the transfer of data from the frontend electronics to the main acquisition computer. The SBCs in the QT crates transfer the photomultiplier tube pulses into local memory with a block DMA (direct memory access) transfer over the VME bus. Each SBC zero-suppresses the data. The zero-suppressed data is then handed to a routine which sends it over ethernet to the host computer. The host computer accepts data from all the SBCs. When data arrives it is stored in temporary buffers until the data from all the SBCs has arrived for a given window. Each time window is collected together and given to another process which does further analysis.

The SBCs are running a version of Linux as the operating system. The work load is divided into two threads of execution. One thread controls the VME bus DMA transfers and zero-suppression. The other task handles shipping the zero-suppressed data over ethernet. The decision to have two threads was made so that the DMA transfer and ethernet transmission can occur simultaneously. This is because the DMA transfer is controlled by the VME chip. The VME chip frees the processor for other tasks during this time.

The host computer also runs Linux. The collection routine is very simple. Sockets are opened to all the SBCs. This signals the SBCs that it is time to take data. Once all the sockets are open the collection routine waits for data with the Unix select system call. This way no time is wasted polling for data when there is none.

Tests on the existing LSND system have shown that a $10 \mu$ s wide window's worth of full detector data can be transfered to the host computer at the full $15 \mathrm{~Hz}$ rate. Planned improvements in both the software and the hardware data communications subsystems (e.g., the ethernet connections between each SBC and the host) should improve this substantially. However, the relevant point to note is that the MiniBooNE experiment can be done with the existing system. 


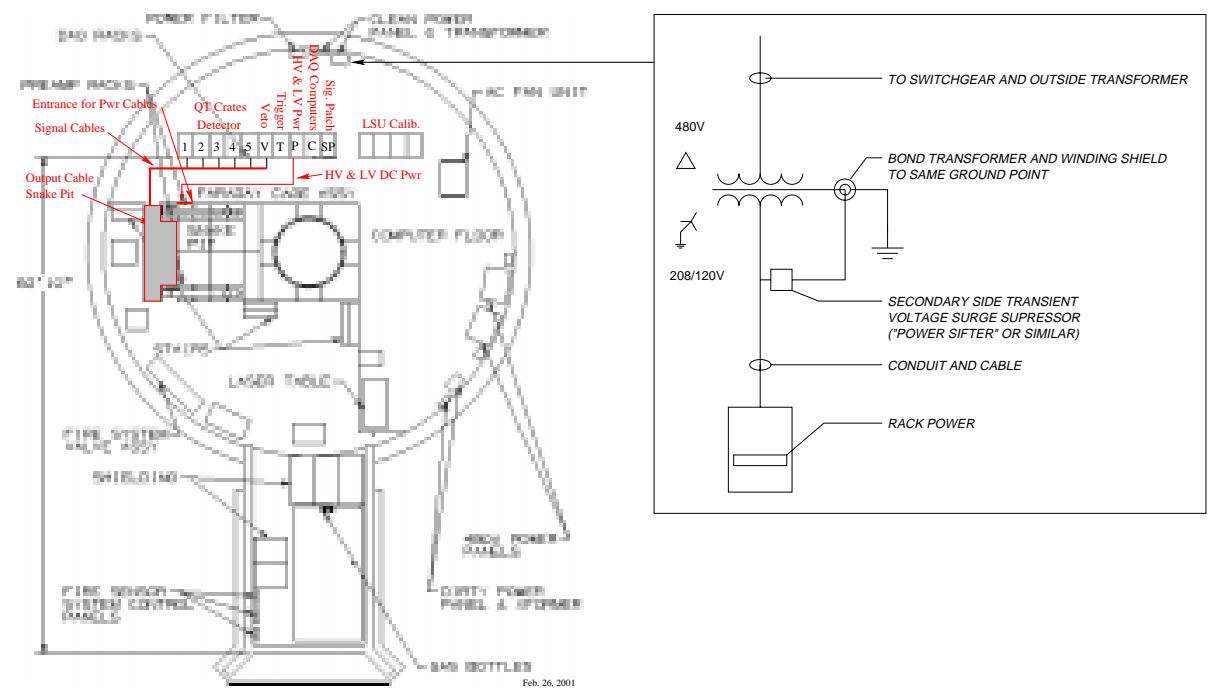

Figure 6.15: Clean and dirty electrical power and cable layout for the MiniBooNE enclosure.

\subsection{Electronics, Signal Paths, and Physical Plant}

The physical layout is shown in Fig. 6.15. The PMT signals run from the tubes to the preamplifiers, which are located above the tank. The amplified signals then travel from the preamps to the electronics area through cables routed in dedicated ducts mounted in the floor. All analog signals are physically separated and isolated from power and digital signal cables. The QT crates, trigger crate, host computer, tape drives, and related electronics are located in the electronics area of the MiniBooNE enclosure. Also located in the electronics area are the low voltage and high voltage power supplies. Power for this area is supplied from a separate transformer winding from the $480 \mathrm{~V}$ distribution transformer and connected to a dedicated 480-to-208/120 V transformer and a "Power Sifter" line conditioner. (The "Power Sifter" is a "TVSS", a transient voltage supression system.)

Chilled and dried air is supplied from the HVAC system through floor level ducts and is directed vertically up through the electronics racks. Fan trays mounted below each electronics crate force air up through the modules. Hot air is exhausted out of the top of the racks and returned through the room. A photograph of the LSND crate system, QT crates, fans, and power supplies, is shown in Fig. 6.16. 


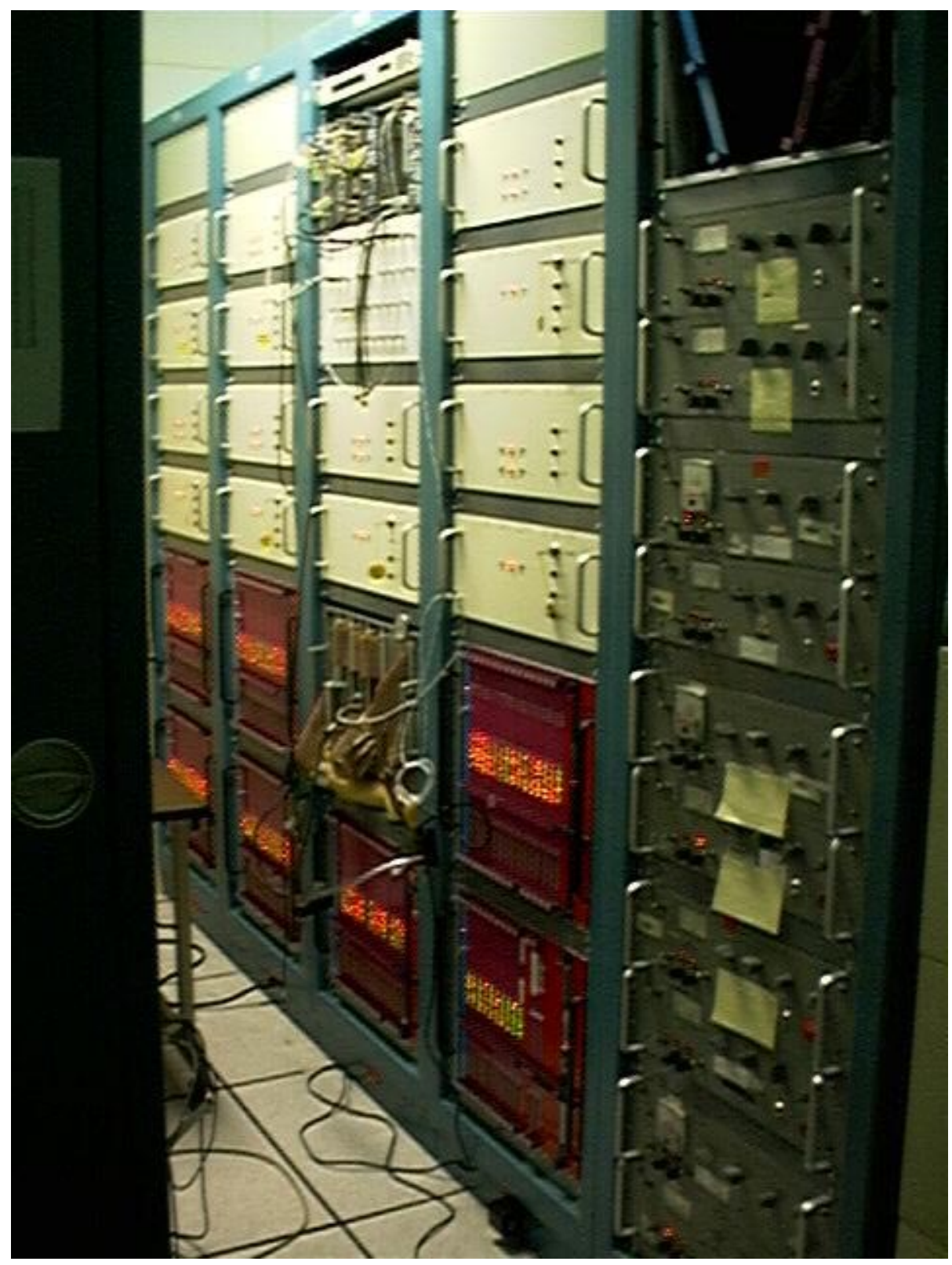

Figure 6.16: A photograph inside of the LSND electronics hut, showing the rack layout of the QT crates and their power supplies. 


\subsection{Appendix 6A: Photomultiplier Tube Bases and Preamplifier Electronics}

The photomultipler base provides the photomultiplier tube with the graded voltages for its dynode chain and couples the fast-current pulse from the anode to the coaxial cable to the front-end electronics. The base used for the LSND PMT, the Hamamatsu R1408 modified, is shown in Fig. 6.3. A simple resistorbased voltage divider provides the voltage taper recommended by Hamamatsu. These values were established after testing by Hamamatsu, SNO, and LSND to optimize gain, collection efficiency, transit time spread, noise, and charge resolution. The base was designed to be robust and stable, as it would be inaccessible for repairs. The design used 1 megohm, $1 / 4 \mathrm{~W}$, metal film resistors of good quality and $1 \%$ tolerance in the divider chain. The total chain resistance was 17 megohms, which draws $118 \mu \mathrm{A}$ when operated at 2,000 $\mathrm{V}$. The maximum voltage across any single resistor was kept below $300 \mathrm{~V}$ and well within power dissipation limits. The large voltage between the photocathode, the collection grids, and the first dynode was developed across a series of 5 and 3 seriesconnected resistors, respectively. The remaining chain is composed of 1 megohm resistors. High voltage ceramic capacitors, with values of $0.01 \mu \mathrm{F}$ and $3 \mathrm{kV}$ ratings, were used to hold voltages stable on the last three dynodes in the lower part of the chain. This provided for better pulse fidelity and gain stability for large amplitude pulses. The parts are mounted on printed circuit card 4.75 inches. in diameter. The traces are run with wide spacings and with layout appropriate for fast-pulse electronics. Plated through holes on the printed circuit card match onto the leads coming from the base of the PMT. The leads are passed through these holes and are soldered directly to the printed circuit card. A single RG-58 coaxial cable was soldered to the base and sealed with a heat shrinkable cable end dressing. This provided good fast-pulse connection and conservative high voltage insulation. The cable was strain relieved by two plastic cable ties threaded through holes in the printed circuit card and around the cable jacket. The base was permanently attached to the PMT at the time of acceptance testing and has stayed with the tube.

A tube and base combination that passed the acceptance testing then had its base and neck dipped into a conformal coating compound. Three coating dippings were applied. This sealed the electrical parts of the base and served to isolate the base from the mineral oil and to protect the mineral oil from the metallic parts of the base.

A similar base has been designed and used on the prototype bases for the Hamamatsu R5912 PMTs used in the "MILAGRO" cosmic ray experiment at Los Alamos and is shown in Fig. 6.4. (The MILAGRO tubes have 10 dynode stages.) The R5912 are available with different numbers of dynodes to offer different gains. MiniBooNE will use a few hundred of these newer tubes with 10 dynode stages. These tubes will use the same preamplifiers as used for the 

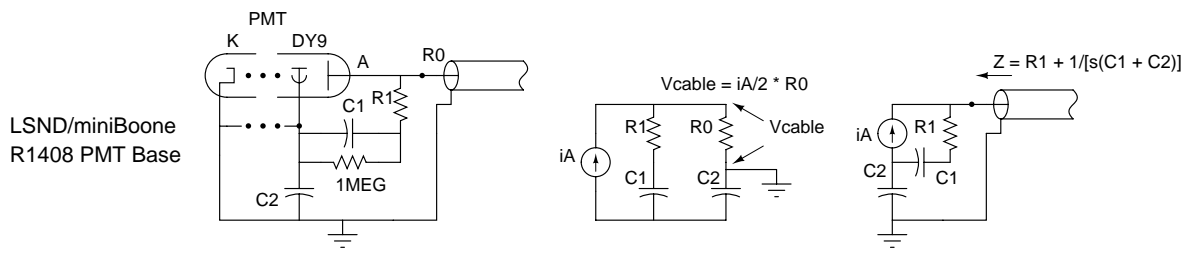

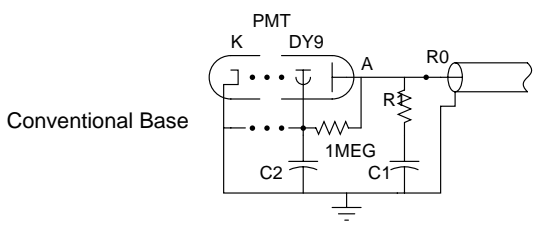

Anode Circuit

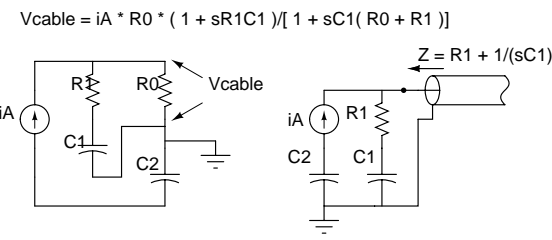

Signal Across Cable
Back Termination Impedance Seen by the Cable

Figure 6.17: The symmetrical back termination design used for the R1408 base. This is compared with a more conventional connection to back terminate a base operated with a single cable.

\section{R1408 tubes.}

The anode circuitry requires some comments. Because the tube is immersed in oil, the photocathode is operated at ground potential to minimize the effects of leakage currents. The tube's anode is therefore at positive high voltage. In order to minimize the amount of inert material in the tank and to simplify the cable plant, a single cable was used to supply both high voltage, typically $2,000 \mathrm{~V}$, and to extract the anode pulse. Approximately 100 feet of RG-58/U $50 \mathrm{ohm}$ coaxial cable connects each PMT to the high voltage distribution and preamplifier electronics. The PMT-end termination circuit is shown in Fig. 6.17. The high voltage to a given tube is set by a pair of resistors in series with the line to the PMT and the common high voltage power supply. The resistors were selected based on the gain verses voltage values determined at the time of the acceptance testing, and were soldered into a preamplifier channel dedicated to the particular tube.

The preamplifier electronics terminates the cable in $50 \mathrm{ohms}$ (in series with the high voltage blocking capacitor) and provides a gain of 20 to the signal before passing it on through another approximately $100 \mathrm{ft}$. of cable to the QT electronics. The PMTs are operated at gains of $4 \times 10^{6}$ to keep their dark count rate below 5,000 counts per second. At this gain the anode pulse corresponding to a single photoelectron is approximately $1.5 \mathrm{mV}$. The preamplifiers raise this to approximately $30 \mathrm{mV}$, which greatly improves the signal to noise in the leading edge discriminators and in the charge integrators in the QT electronics.

The QT charge integrators produce a convolution of the amplified PMT 
pulse with a $1 \mu$ s exponential. This signal is sampled by a FADC every 100 ns and written into the dual port circular buffer memory. Because of this the waveform at the integrator must be reasonably free of reflections between the base and the preamp-end termination. Due to the use of the cable to carry signal and high voltage, a blocking capacitor must appear in series with the terminating $50 \mathrm{ohm}$ resistor and the mismatch results in a small reflection. To reduce this to acceptable levels, the base is back terminated. Again, because of the high voltage on the cable, a network of a series resistor and capacitor is used for the termination. The MiniBooNE bases are configured to take advantage of the high impedance of the anode current source to transmit to the load at the preamp an undistorted image of the anode current pulse. This is illustrated in Fig. 6.17. The "trick" is to arrange the base decoupling capacitors to take advantage of the anode current source by using a symmetrical load of a pair of series resistor-capacitor networks. One of the pairs is the $50 \mathrm{ohm}$ resistor 0.01 $\mu \mathrm{F}$ capacitor that acts as the back termination. The other pair is composed from the $0.01 \mu \mathrm{F}$ decoupling capacitor and the $50 \mathrm{ohm}$ impedance of the cable itself. The common connection at dynode 9 produces this topology. This is illustrated in Fig. 6.17, which compares this symmetric connection to the more common connections of the decoupling capacitors to the common "ground".

(Note: A similar configuration may be employed at the termination end of the cable to mitigate the differentiating effects of the $\mathrm{RC}$ coupling. To analyze this situation, the end of the cable may be modeled as a voltage source of twice the signal voltage in series with its characteristic impedance, in this case 50 ohms. A network of a series capacitor followed by a resistor and another capacitor with value selected to create a symmetric voltage divide produces an undistorted image of the cable voltage at its output. This is illustrated in Fig. 6.18. These networks have the disadvantage of decreasing the value of the series capacitance because the capacitors are in series, reducing their effectiveness as terminations. In practice this is not a problem and the realization of an undistorted anode waveform at the termination end of the cable is a useful result. Eventually, the cable reflections due to the mismatch arising from the capacitors in the terminations corrupts the signal, but at a greatly reduced level and at a time two times the cable delay from when the original pulse arrived. A SPICE simulation confirms these observations.)

The preamp is built from fast current-feedback op amps (AD9617) that are well matched to driving $50 \mathrm{ohm}$ lines. The circuit is shown in Fig. 6.6. Each preamp is an independent amplifier, isolated from all other amplifiers by isolating the local ground plane from the system ground through $100 \mathrm{ohm}$ resistors. The PMTs are "floating" electrically in the tank (i.e., the shield on the cable at the PMT is not connected to the tank wall) and final connection to the "ground" is made at the QT electronics. (This is not a safety hazard, as the return paths for any high voltage is through the safety $100 \mathrm{ohm}$ isolating resistors and at no time are hazardous voltages present at connections that can be accessed by personnel in the QT electronics.) Protection from shorts or other 

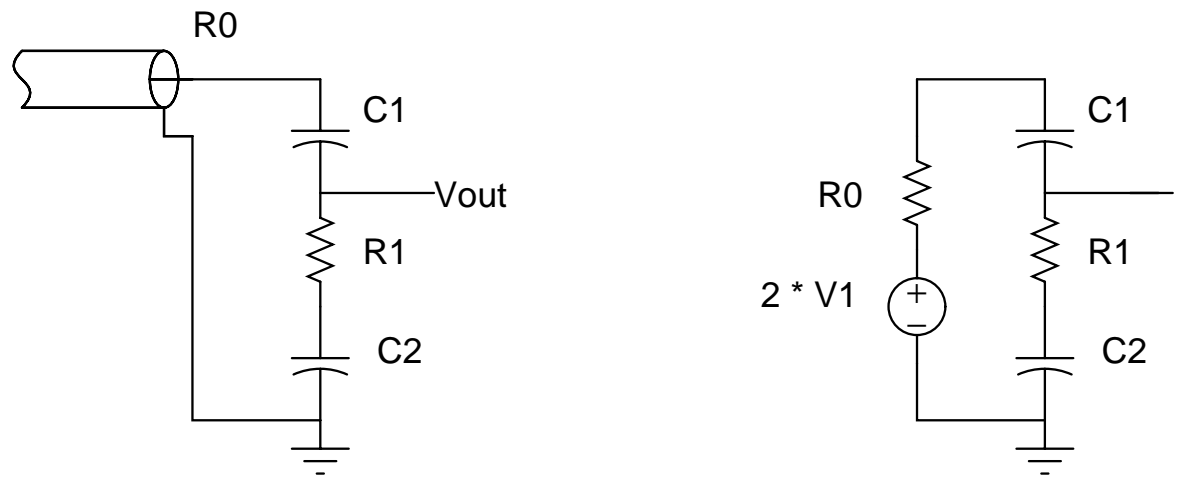

Figure 6.18: A symmetric preamp-end cable termination that avoids the effect of the RC differenation due to the blocking capacitor used in a single cable system.

cable faults that could produce a high voltage spark at the preamp is provided by the diode-resistor series clamps on the front end. The preamps have survived testing with a full charged cable discharged to ground while connected to the operating preamp. They have also survived 7 years of operation by personnel of various degrees of instrument operation experience.

The preamp system is packaged on $9 \mathrm{U}$ x $400 \mathrm{~mm}$ Eurocard format G10 epoxy fiberglass printed circuit cards. The gain setting resistors were soldered into the appropriate channels and the boards were coated with high voltage insulating varnish ("Glyptal"). A single card holds 24 preamp channels. All PMT signal connections are made from the backs of the mounting crates through connector blocks fabricated with SHV connectors for the PMT inputs and through "coaxicon" RF connectors in NIM style "blue connector" blocks. The high voltage connection from a fixed voltage supply is made through a SHV connector on the front panel. Ten preamp cards fit into a standard $9 \mathrm{U}$ crate. The complete system occupies 9 crates. The fixed supplies are connected to the cards through a distribution box located above the crates. This allow for simple apportionment of the load current amongst a bank of fixed output high voltage power supplies. (Typical power supplies include the Bertan 310R-03 fixed voltage supplies.) We plan on using 10 high voltage power supplies and loading them to less than half their maximum rated output current to insure good longevity and reliability.

\subsection{Appendix 6B: QT-Electronics}

The QT electronics provides the front-end PMT pulse processing that creates two analog outputs, one proportional to the integrated charge in the pulse as 
a function of time and the second a linear voltage ramp that begins at a fixed threshold (corresponding to between $1 / 5$ and $1 / 4$ of a photoelectron, the variations due to the gain mismatches amongst the tubes) on the leading edge of the PMT pulse. The time series illustrating these waveforms is shown in Fig. 6.9, the front-end circuit schematic is shown in Fig. 6.19, and the schematic of the digital and VME circuitry is shown in Fig. 6.20. The FADC samples at $10 \mathrm{MHz}$ to 8-bit accuracy. In the case of the time voltage ramp the eight bits resolves the analog signal into 255 channels and allowing for 5 channels of offset from zero and full scale gives a time resolution of $0.8 \mathrm{~ns}$. In the case of the charge, a calibration of $8 \mathrm{ADC}$ channels equals 1 photoelectron of charge constrains the dynamic range to be approximately 30 photoelectrons full scale.

The front-end encodes the data of interest to the experiment; namely, charge and fine time information, in analog voltages that are accurately digitized by the FADC. The system does not attempt to record the PMT waveform. A transient digitization would require a larger sampling rate to meet the Nyquist criteria for good pulse fidelity, and would present an enormous computational burden on the downstream DAQ computers. Our more modest approach gives us the charge ("Q") and time ("T") of a series of arriving PMT pulses in a time series spaced at $100 \mathrm{~ns}$ intervals.

Any PMT pulse arriving is terminated in $50 \mathrm{ohms}$ and buffered by an emitter follower. The input to any channel may be observed through the monitor which is connected to the input through a series $450 \mathrm{ohm}$ resistor. This port may also be used to inject a test pulse to test the particular channel of QT electronics. The buffered signal is sent to the charge integrator and the discriminator.

The integrator circuit is a common base stage. The PMT signal is applied to the gain determining resistor connected to the emitter of the $2 \mathrm{~N} 5770$ transistor. The PMT pulse current (equal to the PMT signal voltage divided by the value of the gain determining resistor, as the transistor's emitter may be considered as a virtual ground to this approximation) is transfered by the transistor to its collector circuit. The collector acts as a current source driving charge onto the $100 \mathrm{pF}$ integrating capacitor (a silver mica low leakage, low inductance capacitor). The voltage across the capacitor (called Vq in Fig. 6.9) is proportional to the charge in the PMT pulse. It is buffered by the 2N4416 FET (configured as a source follower). The FET's source voltage is amplified by an AD829 low noise bipolar op amp, which in turn drives the MC10319 FADC. (Note: These were the best parts available when this system was designed in 1992. Today, in 1999, we would take advantage of subsequent developments and realize improved performance with a smaller parts count. The system described here was built to meet the requirements of good speed and low noise for reasonable costs.) The integrating capacitor is in shunt with the $10 \mathrm{k} \mathrm{ohm}$ collector resistor, which creates an $\mathrm{RC}$ circuit with a time constant of $1 \mu$ sec. Properly speaking, the PMT pulse in convoluted in time with the RC. For sample times of order 100 ns the voltage is close to the integral of the pulse current. For longer times the capacitor discharges and re-zeros its baseline. In this way the digitized time 


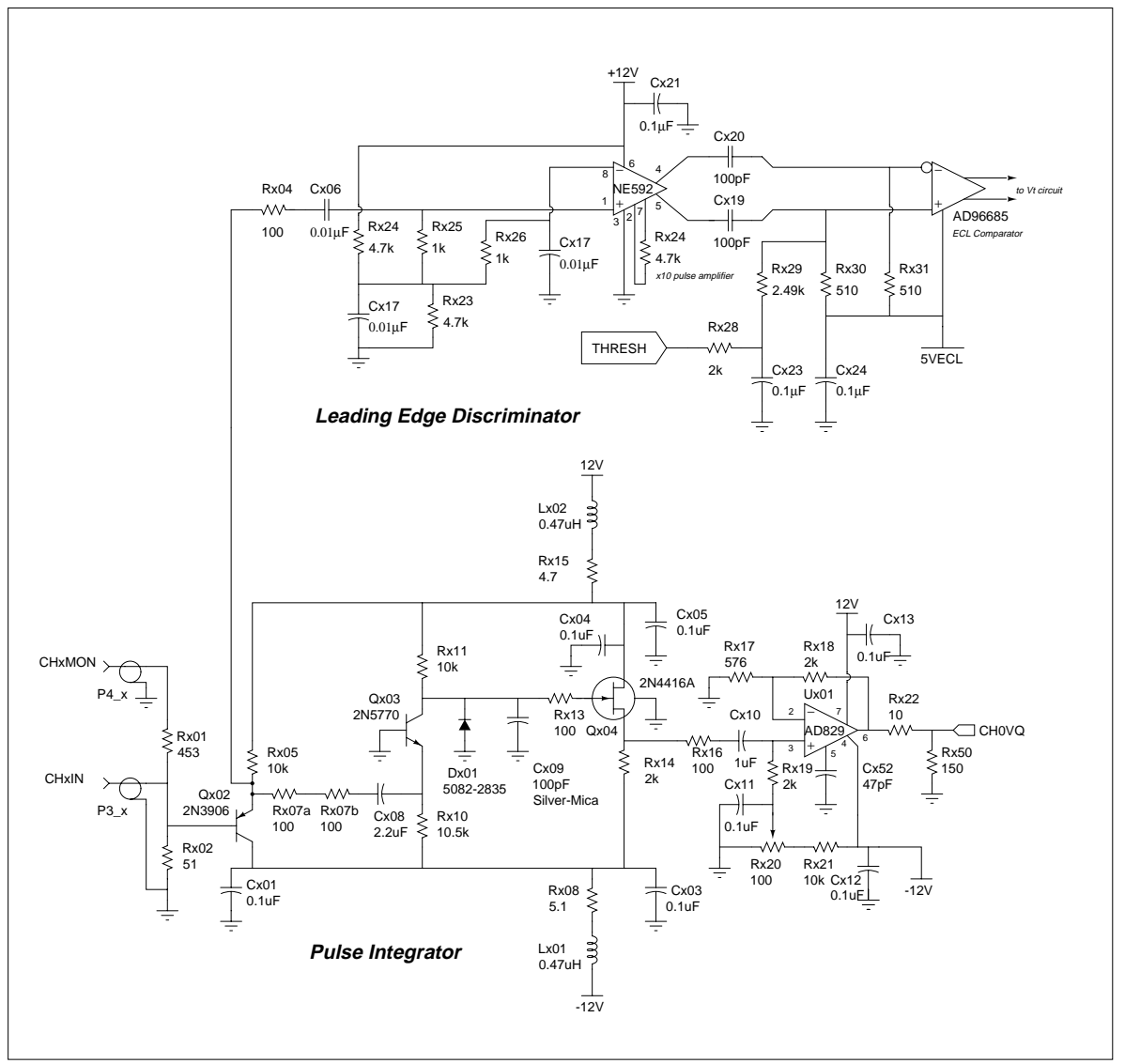

Figure 6.19: The front-end analog electronics for each PMT channel. The upper part of the schematic is the charge integrating circuit. It consists of a common base stage operating as a fast current sink connected to the integrating capacitor. The lower part shows the time interpolating circuit that starts a ramp on the leading edge of the discriminator pulse and resets the ramp on the second subsequent clock pulse. The discriminator threshold is typically set to fire for an input PMT pulse of $-7 \mathrm{mV}$. 


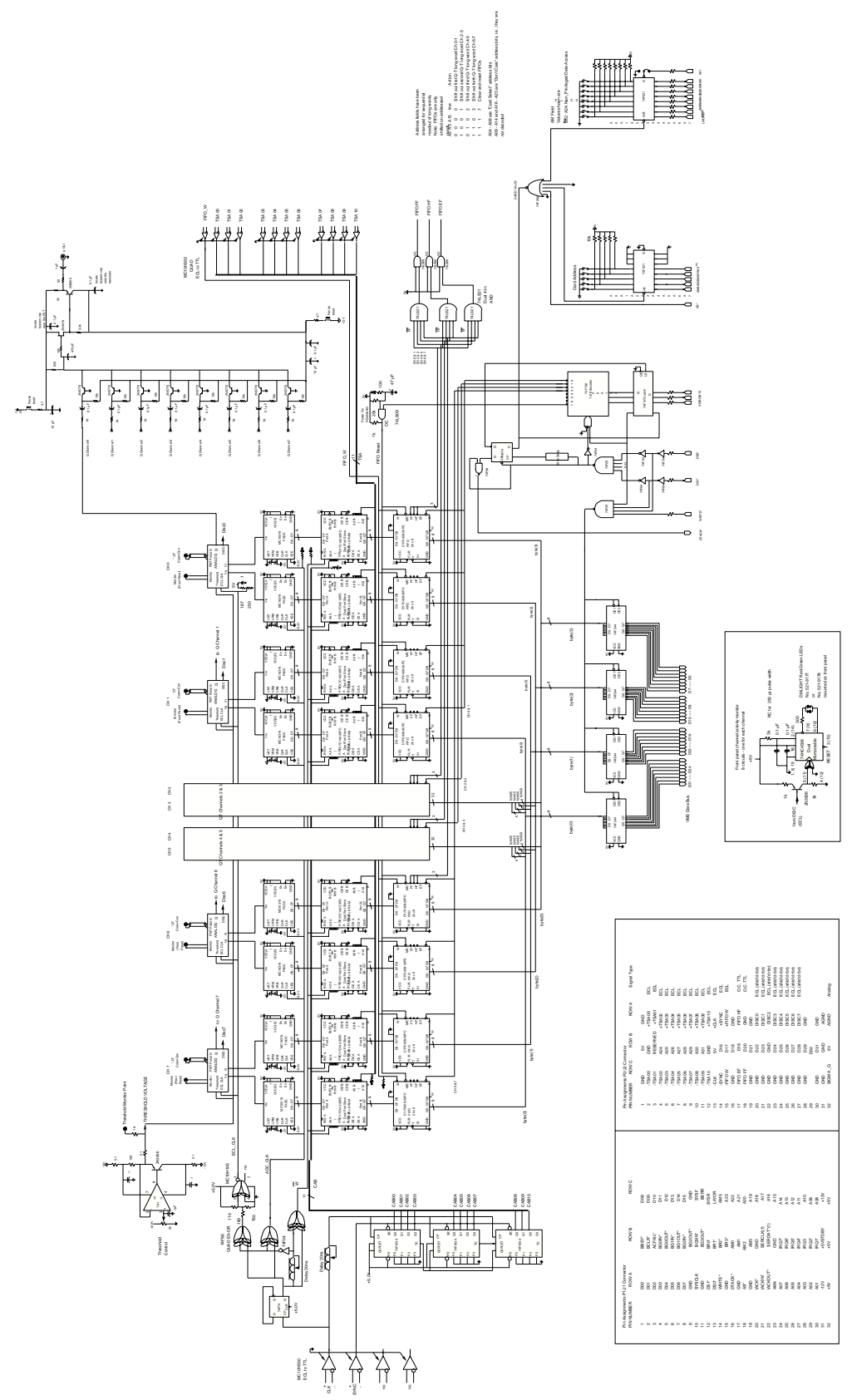

Figure 6.20: The front-end digital electronics on a QT card. The FADCs feed the dual-ported memories, which in turn feed the FIFOs with selected timestamp data. Shown at the bottom of the figure is the VME addres decoding and bus interface logic. 
series of Vq may be read to extract the charge as it flows onto the capacitor without having to gate or reset the integrator.

The discriminator consists of a video amplifier (NE592), a passive RC differenator, a fast ECL-output comparator (AD96685), a set of ECL D-flip-flops, and a stable current source. The PMT pulse is amplified and applied differentially to a fast comparator. Threshold control is provided by offsetting the comparator with a stable DC voltage, Vthreshold. If a pulse exceeds the threshold voltage the digital state is switched. The positive edge transition of this state flips the state of a D-flip-flop, which holds the state until cleared, making the discriminator blind to any subsequent comparator state changes until reset under known conditions.

A time ramp for interpolation of time between the coarse $100 \mathrm{~ns}$ clock ticks is generated from this D-flip-flop. The NPN transistor emitter follower output of the ECL flip-flop is connected to a constant current source with a value of approximately $1 \mathrm{~mA}$. (This value is adjustable by the calibration potentiometer and sets the full scale time range of the time-to-voltage converter.) Also connected to the output of the flip-flop is a $1500 \mathrm{pF}$ capacitor. The voltage across the capacitor is normally "high", which means approximately $+4.2 \mathrm{~V}$. When the flip flop is triggered the state would normally go to "low", which corresponds to $+3.4 \mathrm{~V}$; however, the capacitor holds the voltage up and reverse biases the emitter follower inside of the integrated circuit. This decouples the emitter follower and leaves the charged capacitor to be discharged by the constant current source. The voltage across the capacitor is a linear ramp, denoted by $\mathrm{Vt}$ in fig. 6.9. This voltage is buffered by a second op amp and digitized by a second FADC. The slope and offset are set such that an interval of two clock intervals (200 ns) corresponds to the $2 \mathrm{~V}$ dynamic range of the FADC.

The Vt ramp is started by the PMT pulse and it is stopped just after the second clock tick after the PMT pulse. The FADC samples the Vt at the clock ticks and records the time the pulse arrives with respect to the clock. The two tick window allows the ramp to settle. After the second tick following the pulse, the counter formed from two additional d flip-flops (which are clocked synchronously from the system clock) generates a clear and reset pulse, which resets the first flip-flop and enables the discriminator to accept another pulse. The follower connected to the timing capacitor goes "high" upon reset and pulls up the voltage on the capacitor very quickly (in less than $50 \mathrm{~ns}$ with the values used here). The discriminator can process pulses within 300 ns of one another. (A possible upgrade to the circuit would allow this interval to be reduced to $100 \mathrm{~ns}$ or less if a faster clock is used. It may be used in the future MiniBooNE electronics.)

The digitized $\mathrm{Vq}$ and $\mathrm{Vt}$ data (called $\mathrm{Q}$ and $\mathrm{T}$, respectively) are stored in the dual ported RAM (Cypress CY7C142). The write addresses are generated by a binary counter that counts the $10 \mathrm{MHz}$ system clock. This clock is generated on each QT card from the clock signal distributed on the backplane as a differential PECL signal and is driven by the RCVR card. The counters are synchronized 
with a pulse that resets the counters to zero every $2 \times 10^{12}$ counts. This also is derived from the common $10 \mathrm{MHz}$ clock, distributed to the RCVR cards from the clock in the trigger crate. The on-card binary counter's 11-bit output completes a cycle every 2048 counts or every $204.8 \mu$ sec.

The FADC-to-dualport loop runs continuously, independent of the trigger, and provides a $204.8 \mu$ s history of the PMT activity. The next loop to be described controls the transfer of data from the circular buffer to a FIFO. This loop is controlled by the broadcast card in the trigger crate. The broadcast card transmits FIFO write commands and memory addresses to the RCVR cards. The RCVR card contains a RCVR FIFO that records the time-stamp addresses and other header data and sends the write signal and addresses to the QT cards where they are connected to the dual-ported memories and coordinate the writing of the memory data to adjacent FIFOs (Cypress CY7C428). There it is held until read out by the crate's single board computer (SBC) (Motorola MVME167).

The crate SBC poles a status register in the RCVR card that registers the state of the RCVR FIFO's "empty flag". When the SBC detects that this FIFO is not empty it loops to a service routine that reads out the RCVR FIFO and the subsequent line of data in each of the QT FIFOs. The address space of the QT crate is so arranged that these addresses are sequential, allowing for efficient transfer of data organized with common time stamps. Once inside the SBC the data is processed for $\mathrm{Q}$ and $\mathrm{T}$ information, packaged into ethernet packets, and sent to the host computer.

The system is built in VME format and preserves the VME-Rev. C recommended practices and coding conventions. The boards will operate at significantly faster bus cycle times than the MVME167, which take 200ns per VME bus cycle.

\subsection{Appendix 6C: Commands and Data Struc- tures for the QT and Trigger Cards}

This Appendix contains a listing of the LSND Data Acquisition System Commands. The codes are the addresses of the various commands on the QT Cards, the RCVR Cards, the Broadcast Card, and the Trigger-Memory Card. By either reading from or writing to these addresses the listed function or action is executed. Do not use these commands without a full understanding of what you are doing or you may cause serious harm.

These commands may be executed by hand when in a VxWorks shell and logged on to a monoboard computer. (Current usage is to call these single board computers (SBC). Motorola has coined the term "monoboard computers" (MBC) and we use this interchangeably with the SBC name.) 


\subsubsection{RCVR Card Introduction}

The RCVR Card (receiver card) takes timestamps and event identification data off of the TSA bus, stacks the data on its own FIFO, and sends the TSA data to the QT Cards in the crate (i.e., it drives the local TSA backplane located on the P2/J2 connectors). For purposes of read out during data acquisition mode, only the reset command, "status register" read command and the FIFO read command are of interest. The other commands are for testing and diagnostic purposes. (The RCVR Card was designed to supply the necessary test signals to operate a single QT Crate on it own as a test stand to debug QT Cards and Sigma PMT Cards.)

\subsubsection{RCVR Card Operation}

The RCVR Card is designed to be the first card in a package of cards consisting of: MBC (MonoBoard Computer) (card -1, if you will), RCVR Card (card 0), QT Card 1, QT Card 2, . . ., QT Card N (usually 16), and the Sigma PMT Card (which terminates the TSA backplane and sums the DISC bits into an 8-bit wide binary sum). The RCVR Card and the bundle of QT Cards are arranged to be contiguous in their address space. This allows for efficient readout and minimizes both local bus and VME bus access time through the use of several modes of the VMS-CHIP2 in the MVME167 MBC.

The VME bus is set to operate as: D32: A24: Non-Privileged Data Access. The AM fields (Address Modifier fields) on the decoder logic on the RCVR and QT Cards are set to issue Card Valid signals for this class of address access only. However, to save circuit card real estate and costs, not all address bits in the A24 field are decoded. Address bits A02 - A08 and A15 are decoded. Bits A09 A14 and A16 - A23 are not decoded and if addressed, with the appropriate lower bits set, they will cause the same actions as if they were zero. The A15 bit is used to distinguish test and control commands from the more routine reading of the status register and the FIFO. This makes the definition of a "page" a little mixed up. In future versions we will decode the full address space. In practice, 16 QT Cards fill up a crate, so there is no real limitation in this somewhat sloppy usage.

The address modifier (AM) decoders on the cards are set to the following values for D32:A24:Non-Privileged VME bus transactions:

$\begin{array}{lll}\text { AM0 } & \text { H } & 1 \\ \text { AM1 } & \text { L } & 0 \\ \text { AM2 } & \text { L } & 0 \\ \text { AM3 } & \text { H } & 1 \\ \text { AM4 } & \text { H } & 1 \\ \text { AM5 } & \text { H } & 1\end{array}$




\subsubsection{RCVR Card Commands and Data Output Format}

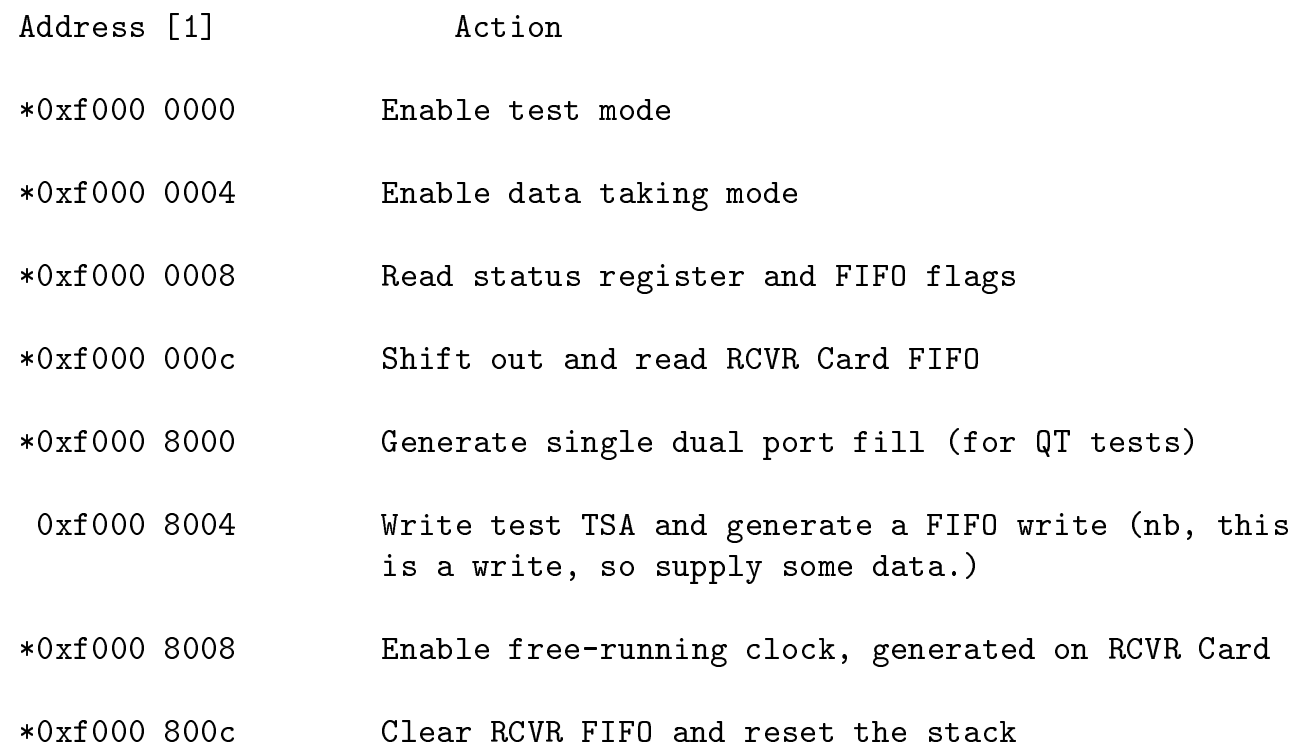

[1] The prefix 0xf000 XXXX is determined by the way the particular MBC's VME_CHIP2 is configured to map to the VME bus. The QT Crate MBCs are set for D32:A24:Non-privileged VME bus operation.

Example 1: To read the status register, the c code

$$
\mathrm{x}=* 0 \mathrm{xf000} 0008
$$

will return the contents of the status register in the variable $\mathrm{x}$. $\mathrm{x}$ should be a long (32-bit) unsigned integer.

Example 2:

The following c code will write a test TSA to the RCVR Card:

$$
* 0 \times f 0008004=0 x a
$$

This writes the bit pattern 0000000000001010 to the RCVR Card.

Data Formats

Issue a $* 0 x f 000000 \mathrm{c}$ to shift out and read RCVR Card FIFO puts data out on the VME bus in the following Long Word format: 


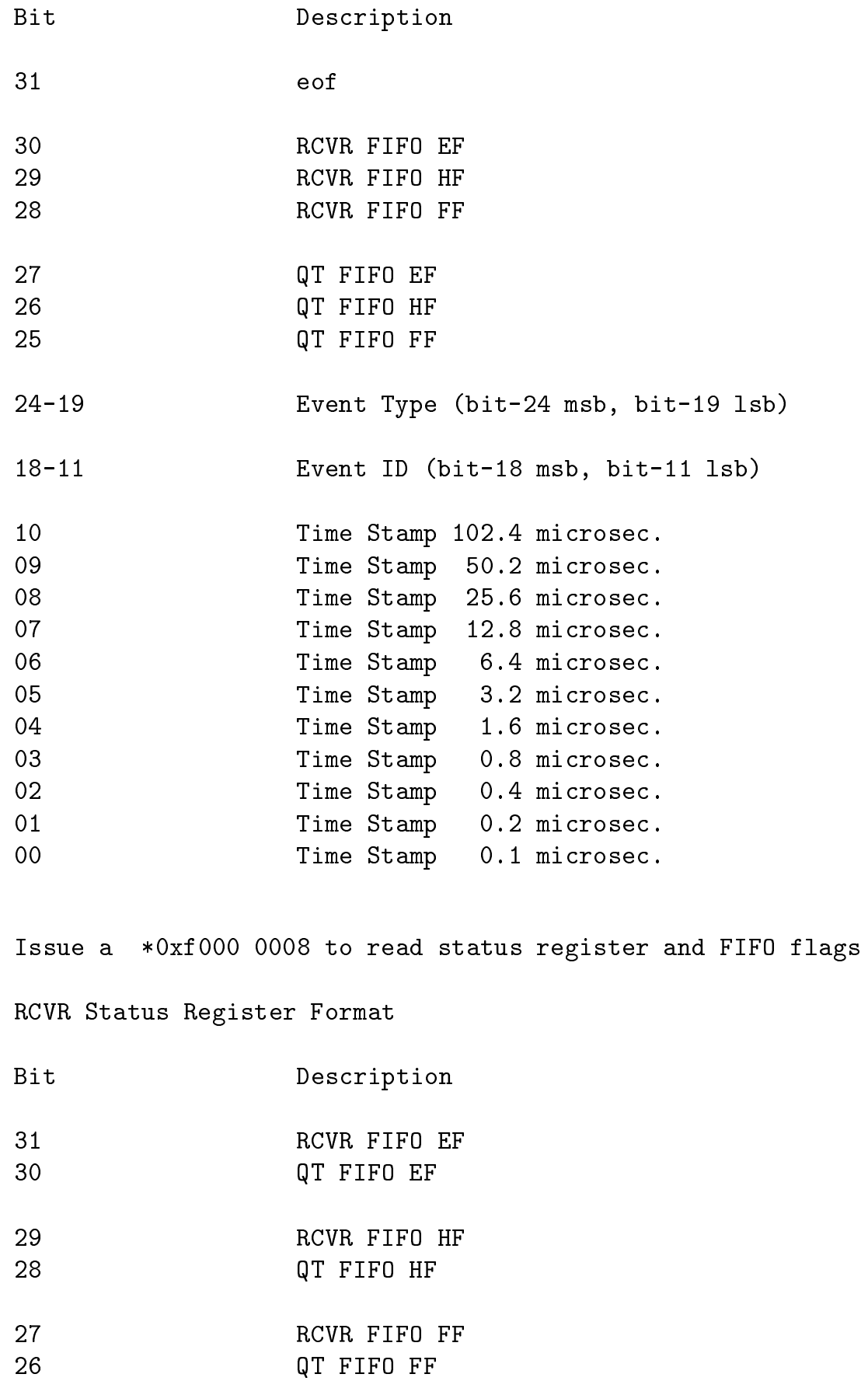


Issue a 0xf000 8004 to write test TSA and generate a FIFO write (nb, this is a write, so you must supply some data in the format shown below.) RCVR Card must be in Test mode to use this command.

$\begin{array}{ll}\text { Test TSA Write Data Format } \\ \text { (VME) Data Bit } & \text { Description } \\ & \\ \text { D00 } & \text { TSA 00 } \\ \text { D01 } & \text { TSA 01 } \\ \text { D02 } & \text { TSA 02 } \\ \text { D03 } & \text { TSA 03 } \\ \text { D04 } & \text { TSA 04 } \\ \text { D05 } & \text { TSA 05 } \\ \text { D06 } & \text { TSA 06 } \\ \text { D07 } & \text { TSA 07 } \\ \text { D08 } & \text { TSA 08 } \\ \text { D09 } & \text { TSA 09 } \\ \text { D10 } & \text { TSA } 10\end{array}$

\subsubsection{QT Card Commands}

The QT Cards use A04 - A08 as "Card Select Lines" and jumper fields on the card are set to select a particular combination to set the card's address. This provides for unique addresses for 1 to 16 cards. (Actually for 1 to 31 cards, since five bits are used.) Address lines A02, A03, and A15 are used to select the particular on-card function. These are defined below. In order to save money, but not grief, it was decided to use programming discipline and beg people not to address anything that would use address lines A09, A10, A11, A12, A13, A14, or A16 - A23. (Note: The QT Crates operate in the world of D32: A24: Non-Priv. Data Access.)

On Card Addresses:

Address Action 
|

I

ํ.

ก้อ

Iี

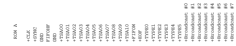

|

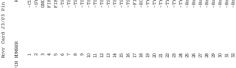
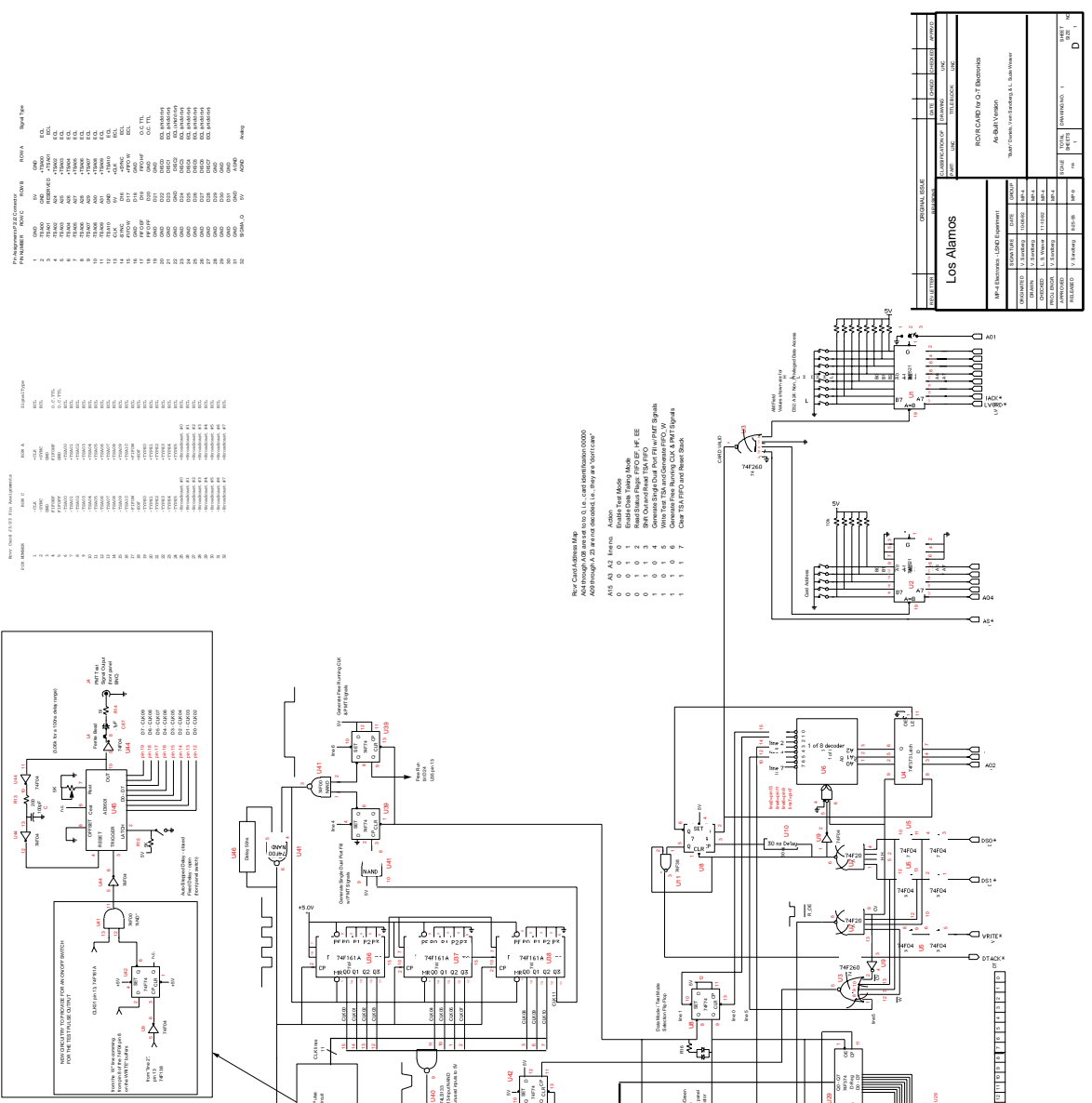

$+1$
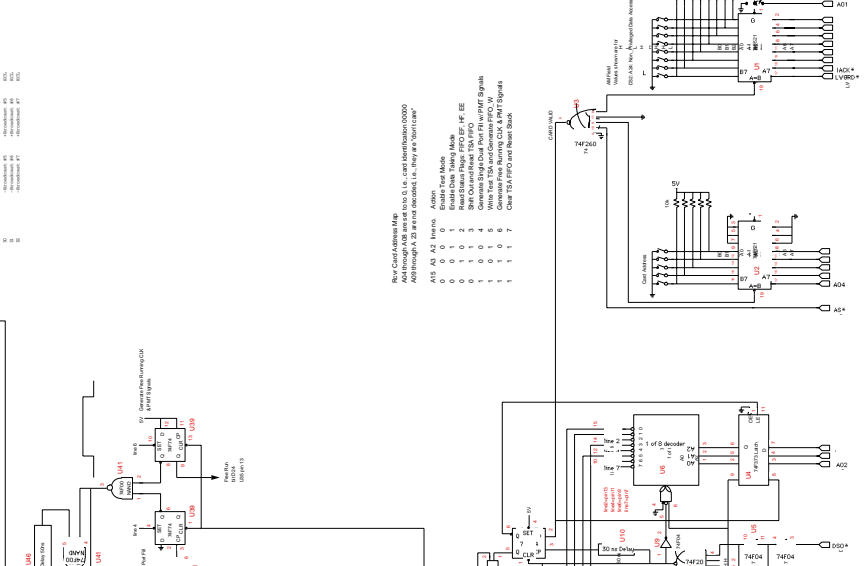

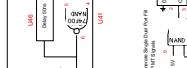

(1)
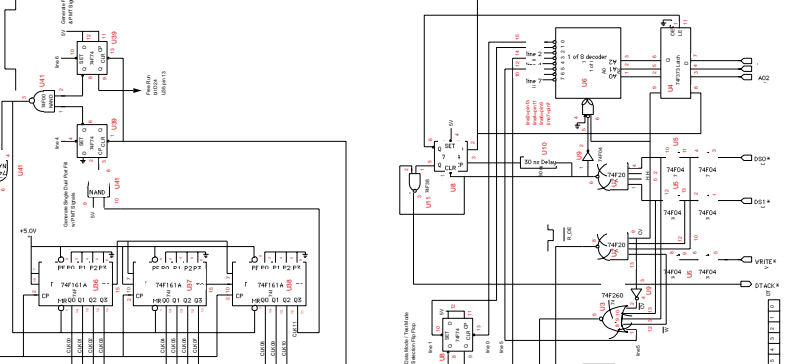

1.
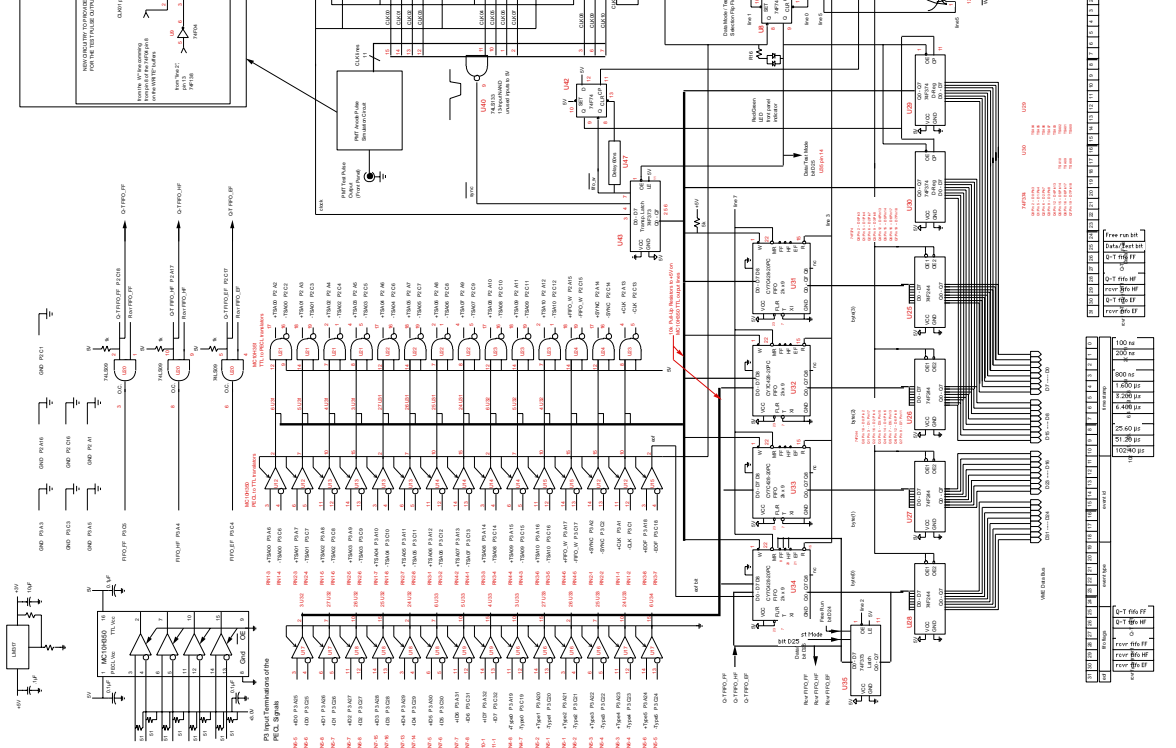

Figure 6.21: The LSND RCVR Card used to communicate between the QT Crate and the Trigger Broadcast Card. It distributes TSA signals and stacks the broadcast timestamps. 


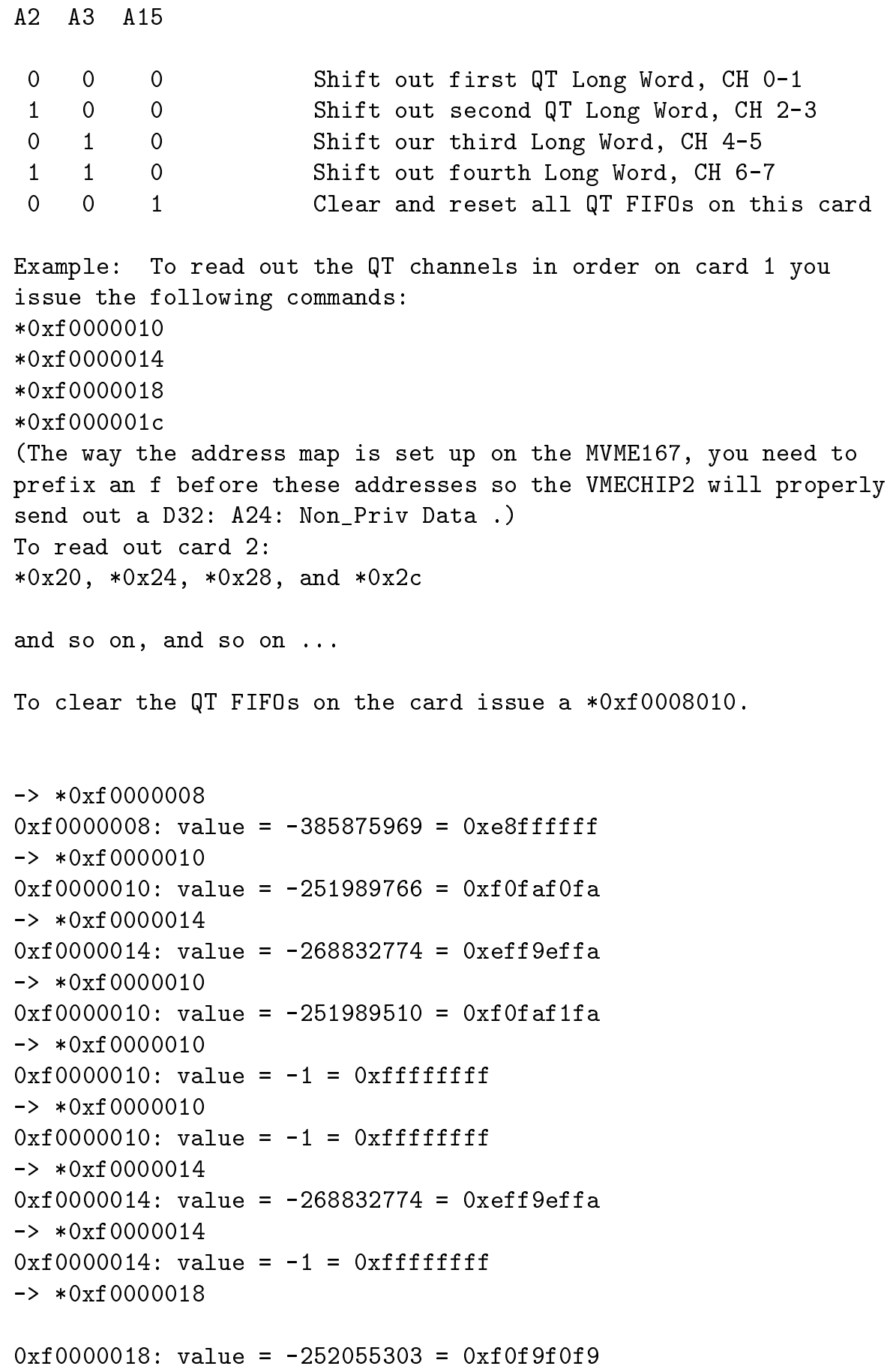




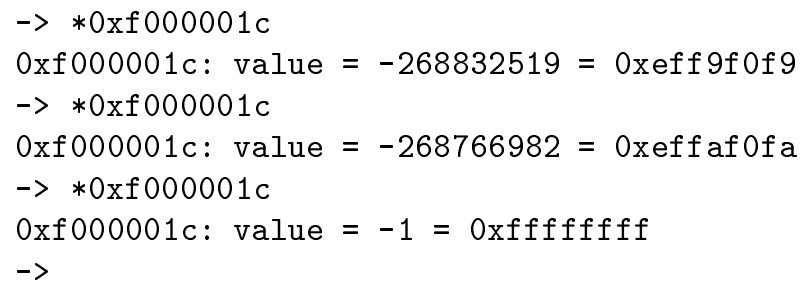

\subsubsection{Broadcast Card Introduction}

The Broadcast Card provides the interface between the trigger MBC and the RCVR Cards. The Broadcast card drives the TSA cable. Its primary purpose is to broadcast the time stamp addresses selected by the trigger MBC. It receives the base TSA and the number of sequential TSAs to be broadcast from the trigger MBC. It then sends out a series of sequential TSAs along the TSA Cable. Upon transmission of the final TSA, an EOF bit is sent and the state of the trigger's binary clock is recorded in a separate FIFO in the Broadcast Card (called the diagnostic FIFO). The trigger MBC should read this FIFO to determine when the last TSA in a molecule was sent to determine if the data was stale.

As with the RCVR Card, the VME bus transactions are D32: A24: NonPrivileged Data. The Broadcast Card decodes the full A24 address space.

\subsubsection{Broadcast Card Commands and Data Formats}

Commands

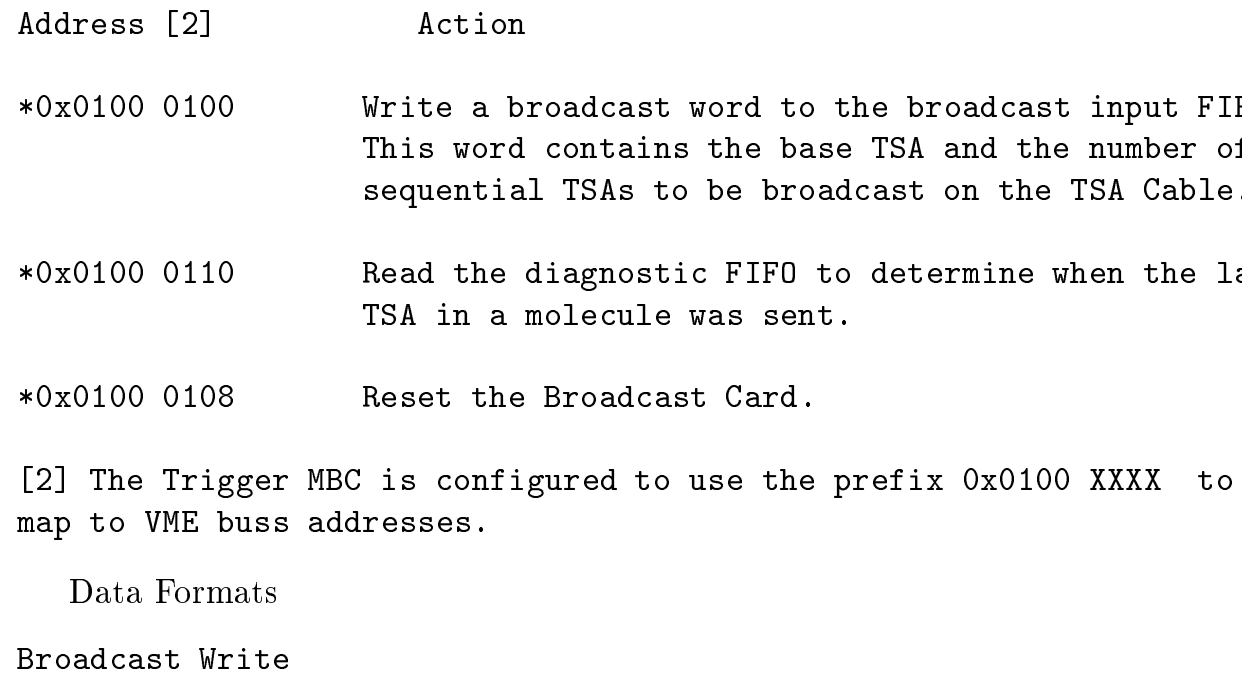




\begin{tabular}{|c|c|}
\hline Bit & Description \\
\hline 31 & Broadcast Identification 7 (msb) \\
\hline 30 & Broadcast Identification 6 \\
\hline 29 & Broadcast Identification 5 \\
\hline 28 & Broadcast Identification 4 \\
\hline 27 & Broadcast Identification 3 \\
\hline 26 & Broadcast Identification 2 \\
\hline 25 & Broadcast Identification 1 \\
\hline 24 & Broadcast Identification 0 (lsb) \\
\hline 23 & Event Type 5 (msb) \\
\hline 22 & Event Type 4 \\
\hline 21 & Event Type 3 \\
\hline 20 & Event Type 2 \\
\hline 19 & Event Type 1 \\
\hline 18 & Event Type 0 (lsb) \\
\hline 17 & not used \\
\hline 16 & not used \\
\hline 15 & Window size N4 (msb) \\
\hline 14 & Window size N3 \\
\hline 13 & Window size N2 \\
\hline 12 & Window size N1 \\
\hline 11 & Window size NO (lsb) \\
\hline 10 & TSA 10 (msb) \\
\hline 09 & TSA 9 \\
\hline 08 & TSA 8 \\
\hline 07 & TSA 7 \\
\hline 06 & TSA 6 \\
\hline 05 & TSA 5 \\
\hline 04 & TSA \\
\hline 03 & TSA 3 \\
\hline 02 & TSA 2 \\
\hline 01 & TSA 1 \\
\hline 00 & TSA 0 (lsb) \\
\hline
\end{tabular}

Diagnostic Read

Bit

Description 


\begin{tabular}{|c|c|}
\hline 31 & Broadcast Identification 7 (msb) \\
\hline 30 & Broadcast Identification 6 \\
\hline 29 & Broadcast Identification 5 \\
\hline 28 & Broadcast Identification 4 \\
\hline 27 & Broadcast Identification 3 \\
\hline 26 & Broadcast Identification 2 \\
\hline 25 & Broadcast Identification 1 \\
\hline 24 & Broadcast Identification 0 (lsb) \\
\hline 23 & $80 \mathrm{msec}$. bit (note: GPS data are in BCD) \\
\hline 22 & GPS Clk $40 \mathrm{msec}$. bit \\
\hline 21 & GPS Clk 20 msec. bit \\
\hline 20 & GPS Clk $10 \mathrm{msec}$. bit \\
\hline 19 & $8 \mathrm{msec}$. bit \\
\hline 18 & $4 \mathrm{msec}$. bit \\
\hline 17 & $2 \mathrm{msec}$. bit \\
\hline 16 & $1 \mathrm{msec}$. bit \\
\hline 15 & Binary Clk 3276.8 microsec. bit \\
\hline 14 & Binary Clk 1638.4 microsec. bit \\
\hline 13 & Binary Clk 819.2 microsec. bit \\
\hline 12 & Binary Clk 409.6 microsec. bit \\
\hline 11 & Binary Clk 204.8 microsec. bit \\
\hline 10 & Binary Clk 102.4 microsec. bit \\
\hline 09 & Binary Clk 51.2 microsec. bit \\
\hline 08 & 25.6 microsec. bit \\
\hline 07 & 12.8 microsec. bit \\
\hline 06 & 6.4 microsec. bit \\
\hline 05 & 3.2 microsec. bit \\
\hline 04 & 1.6 microsec. bit \\
\hline 03 & 0.8 microsec. bit \\
\hline 02 & 0.4 microsec. bit \\
\hline 01 & 0.2 microsec. bit \\
\hline 00 & 0.1 microsec. bit \\
\hline
\end{tabular}

\subsection{Appendix 6D: Communication Cable Tim- ing Measurements}

\subsubsection{Introduction}

The logging of the timing of the beam extraction from the Booster must be recorded by the MiniBooNE DAQ QT electronics to coordinate beam and detector data. The physical separation of the MiniBooNE electronics area from the 
Booster extraction system requires the use of relatively long cables. In order to achieve this synchronization of Booster beam extraction time (or the time when the beam hits the target) with events in the detector, we need to accurately measure the propagation delay in the cable connecting the beam electronics to the MiniBooNE electronics. The cable connecting the MiniBooNE electronics to the Booster signal generating electronics will be $500 \mathrm{~m}$ to $1 \mathrm{~km}$ long and have a propagation delay on the order of microseconds. The propagation delay must be measured and logged along with the times of arrivals of the accelerator and beam extraction signals. Ideally, the cable delay and the timing signal measurements would take place in the same time window.

The cables will necessarily be located in an outdoor environment and changes in temperature will affect a cable's propagation time, and not necessarily in ways you might at first imagine. The most obvious effect is the change in length with temperature. This effect has a positive temperature coefficient; namely, for a positive change in temperature there is an increase in length of cable and a corresponding increase in delay time. But, there is also a reduction in the velocity of a pulse in the cable due to the change in dielectric constant with temperature and this effect has a negative temperature coefficient; namely, for an increase in temperature the delay is reduced, the pulse velocity increases, and the propagation delay decreases. The effect of the dielectric is usually larger than the increase in length due to thermal expansion. The main lesson to be learned is: MEASURE THE PROPAGATION DELAY AT THE TIME YOU MEASURE THE TIMING PULSE.

\subsubsection{Proposed Solution}

The circuit described in this Appendix records the times of arrival of the Booster extraction and target monitoring pulses at the MiniBooNE electronics and measures the propagation delays in the cable carrying these pulses. The scheme takes advantage of the multi-hit capability of the LSND/MiniBooNE DAQ system by using the reflection of a pulse from a shorted cable termination to record the round trip transit time along the cable. Recorded in the event record are the times of arrival for two pulses, the primary timing pulse and its reflection. The time of the primary pulse is the time the beam was extracted plus cable propagation delay. The time interval between the primary and the reflected pulse is equal to twice the sum of the cable propagation delay and a 100ns offset. Both the primary and reflected pulse times are recorded in the MiniBooNE QT electronics and are available for off-line analysis.

In operation, a time signal at the Booster or the target triggers a current source which drives a long cable between the Booster and the MiniBooNE electronics. A short stub of cable that is shorted at its opposite end is connected in parallel with the main cable. The stub inverts and reflects the pulse. The pulse width and the stub length are selected to produce a clean bipolar pulse with a positive and a negative components, as shown in Fig. 6.22. This pulse travels 
along the long cable to the MiniBooNE electronics, where it is picked off by a high impedance amplifier. The pulse continues down a similar stub of cable to another shorted termination, where it is again reflected and inverted. The width of the pulse and stub are adjusted so that the reflected inverted pulse overlaps with the original pulse and doubles the pulse voltage at the pickoff point. The pulse then continues on down the transmission cable to the accelerator end, where it is reflected and inverted and travels back again to the MiniBooNE electronics. On the return a similar sequence of events takes place; the pulse is reflected and inverted, and the pulses overlap at the pickoff giving a pulse of twice the amplitude.

The losses in the cable greatly reduce the pulse amplitude. This loss effectively limits the number of bounces to two. At the MiniBooNE electronics the signal at the pickoff is amplified, discriminated, and fed into a QT channel for recording. The scheme is simple, records the critical beam timing data, and collects cable propagation time delay information. The use of short circuit clipping stubs produces bipolar signals and leaves no net DC or low frequency backgrounds on the cable.

A simple SPICE model of the idea is shown in Fig. 6.23. A fast current source (i.e., a high output impedance source) is connected to two coaxial cables $\mathrm{T} 1$ and $\mathrm{T} 2$. The pulse width of the current pulse is set to twice the electrical length of cable T1. Pulses begin to travel down cables T1 and T2. After 100ns the pulse in $\mathrm{T} 1$ reaches the shorted end of $\mathrm{T} 1$ and reflects inverted in polarity back towards cable T2. After 200ns from the initial pulse this pulse returns to the T1-T2 junction, where it continues on along T2 towards the T2-T3 junction. The current source has a minimal effect on the pulses due to its high impedance. The voltages at the junctions of T1-T2 and T2-T3 are shown in Fig. 6.25 and Fig. 6.26, respectively. Note that the pulse width and length of $\mathrm{T} 1$ have been arranged to produce a bipolar pulse propagating towards the T2-T3 junction.

At the far end of $\mathrm{T} 3$ the pulse is again reflected by the shorted termination and travels back down the cables towards T1. The voltage seen at the T2T3 junction is a double bumped bipolar pulse. The pulse travels back down $\mathrm{T} 2$ to $\mathrm{T} 1$, is again reflected back towards $\mathrm{T} 3$, and after a delay of the travel time down and back along $\mathrm{T} 2$ and $\mathrm{T} 1$, it appears again at the T2-T3 junction. The interval between these pulses at $\mathrm{T} 2-\mathrm{T} 3$ is just the propagation delay along T2 (aside from the correction due to the shorting stub T1). In this SPICE simulation ideal cables were assumed and the pulses appear to bounce back and forth forever. In practice cable dispersion and resistive losses limit the number of bounces to two.

\subsubsection{Practical Realization}

The system may be realized with conventional NIM modules (e.g., LeCroy or Phillips discriminators with current source outputs). A simple design for the cable plant which is reliable and maintainable is of great importance for critical 


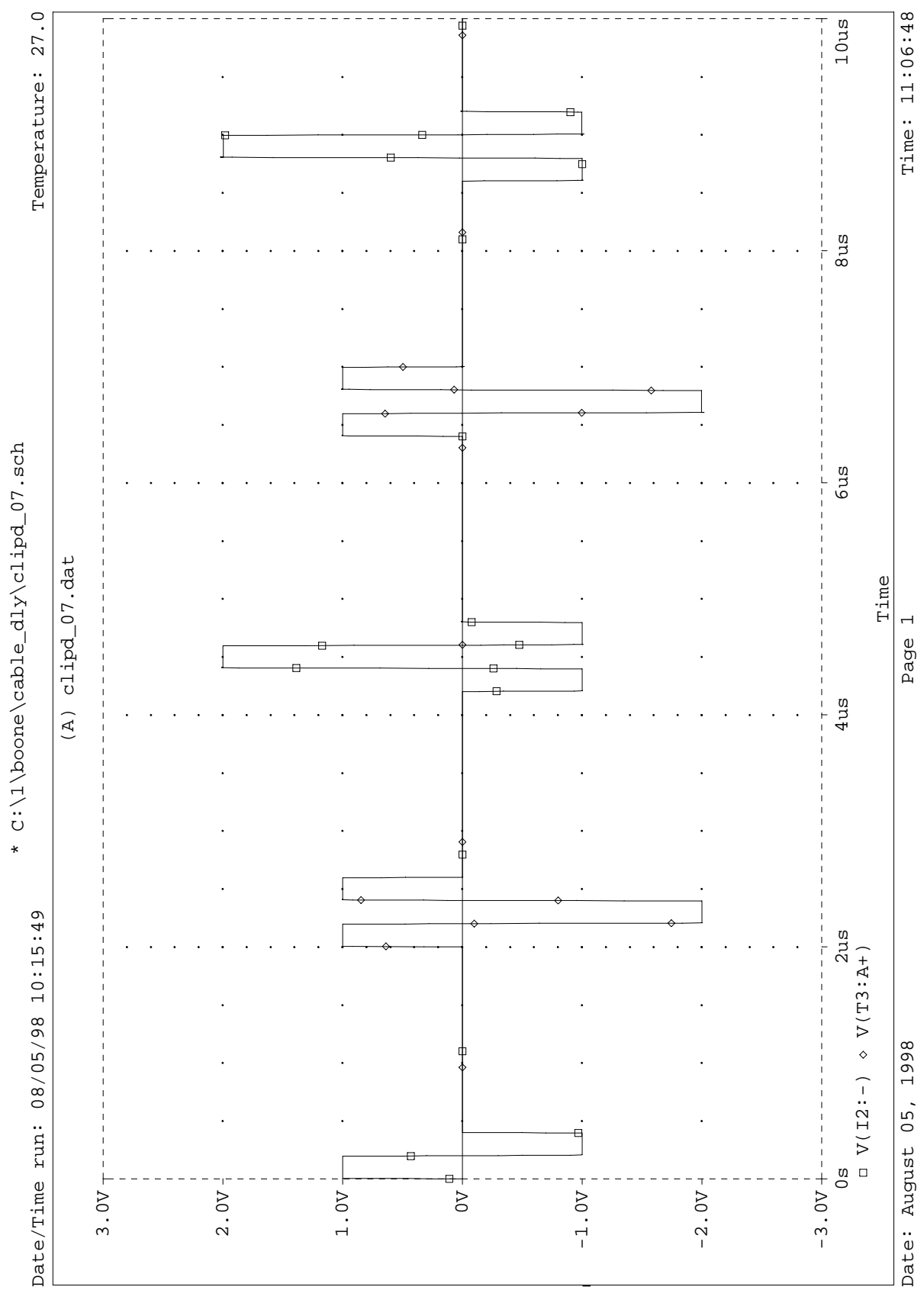

Figure 6.22: Plot of the SPICE simulation of the cable delay timing scheme. Voltages at the T1-T2 and T2-T3 junctions are shown on the traces marked with squares and diamonds, respectively. The plot is shown in landscape mode to allow better resolution of the waveform. 


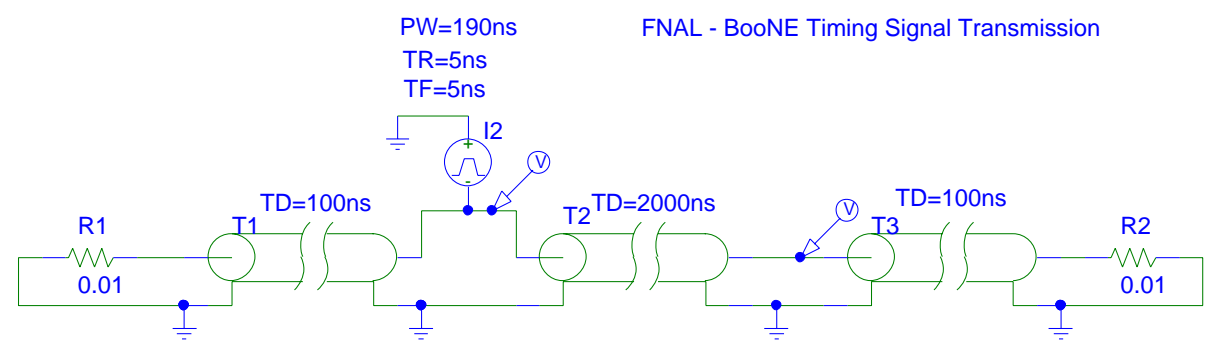

Figure 6.23: schematic of the SPICE simulation.

signals such as the beam timing information. We suggest the use RG-8 or similar high quality coaxial cable for the actual cable runs between accelerator and MiniBooNE electronics. Air core or similar cable is expensive and is not needed here because we are not concerned with the size of the propagation delay, only its measurement and stability.

A model of the proposed system was setup as shown in Fig. 6.24, using $320 \mathrm{~m}$ of RG-223, (which produced a total delay of $1.5 \mu \mathrm{s}$ ) driven by a LRS discriminator. Oscilloscope traces of the pulse trains at the driving point and the pickoff point are shown in Figs. 6.25 and 6.26, respectively. The effects of the cable on the pulse is clear; however, the second pulse is of sufficient amplitude $(100 \mathrm{mV})$ to be reliably detected and discriminated.

\subsubsection{Notes}

Interesting Note 1: The cable clip produces the bipolar pulse and may be used to control the length and height of the pulse as seen at the cable termination points. The driving pulse width can be adjusted to equal twice the delay stub's length (in time). The inverted polarity reflected pulse will sum with the primary pulse and produce an almost symmetrical pulse in the opposite polarity. (The asymmetry arises from the losses and spreading of the pulse by the cable.) At the receiving end, if the receiving end's stub is adjusted to the proper length, then the first part of the arriving pulse will be reflected and superimpose with the second part of the arriving pulse. The net effect is an increase in the pulse amplitude of the second part of the pulse. The increase is not a full doubling, however. The losses and spreading of the pulse as it propagates along the cable produce distortions that reduce the usefulness of this amplitude increase in a practical application.

Interesting Note 2: The main source of pulse spreading and dispersion of pulses in coaxial cables is due to the skin effect of high frequency currents on the outside edge of the inner conductor. The lower the frequency the deeper into the copper cylinder (the center conductor) the current penetrates. Higher frequencies penetrate less deeply. The effective cross sectional area and hence 


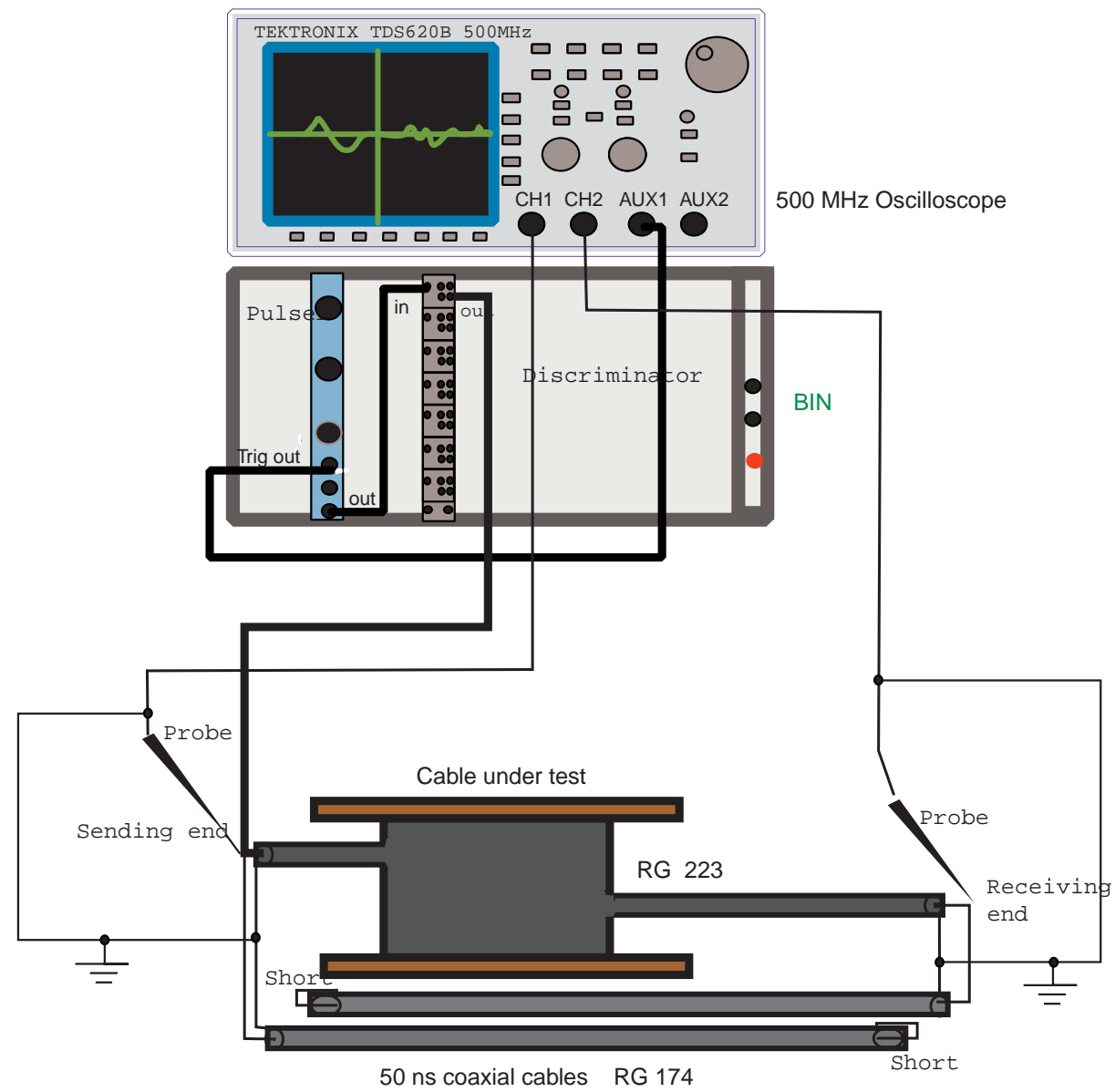

Figure 6.24: Illustration of the test setup. The setup used 320m of RG-223 coaxial cable and 100ns long termination cable stubs at either end. 


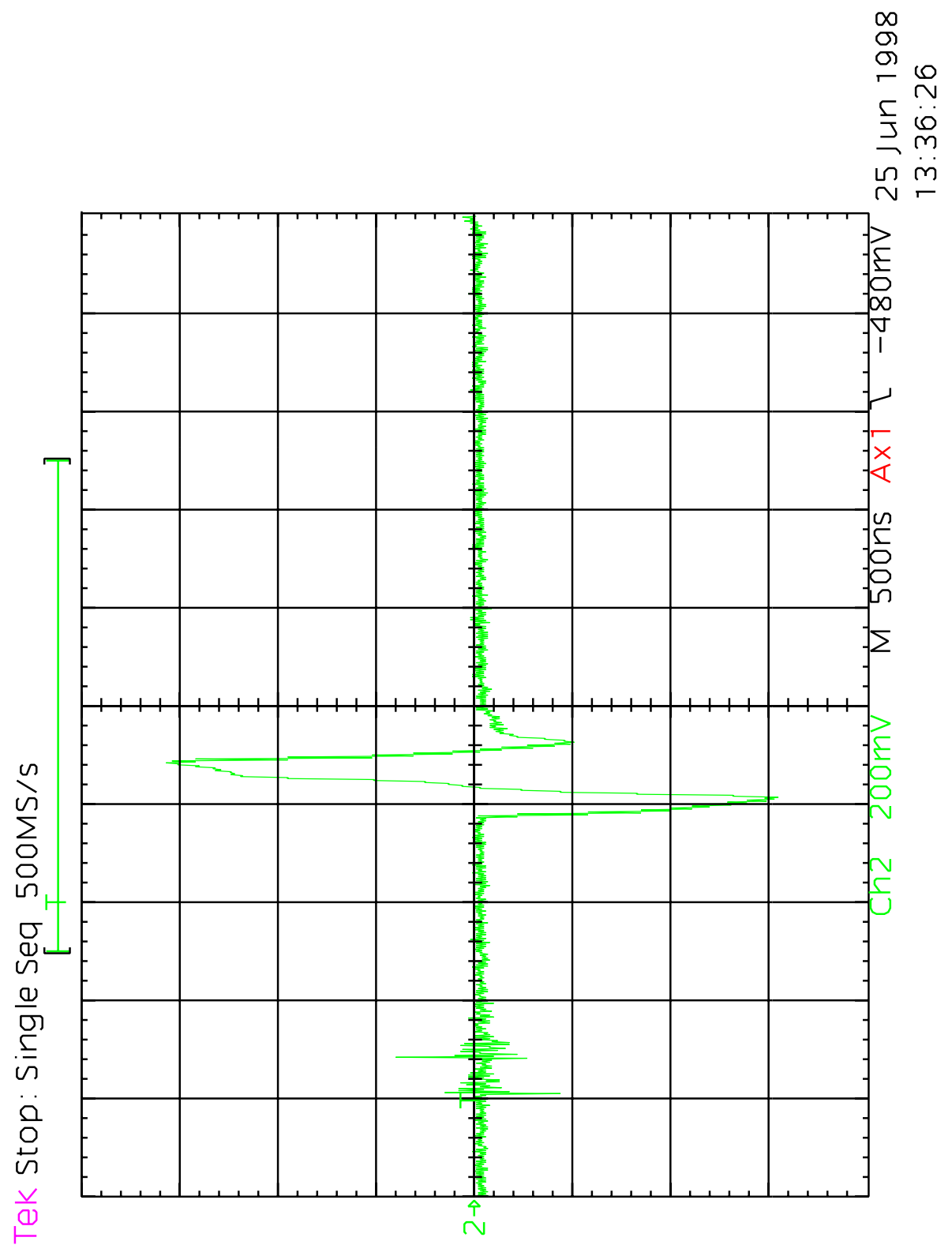

Figure 6.25: Tektronix 620 DSO trace at the driving point (T1-T2 junction). 


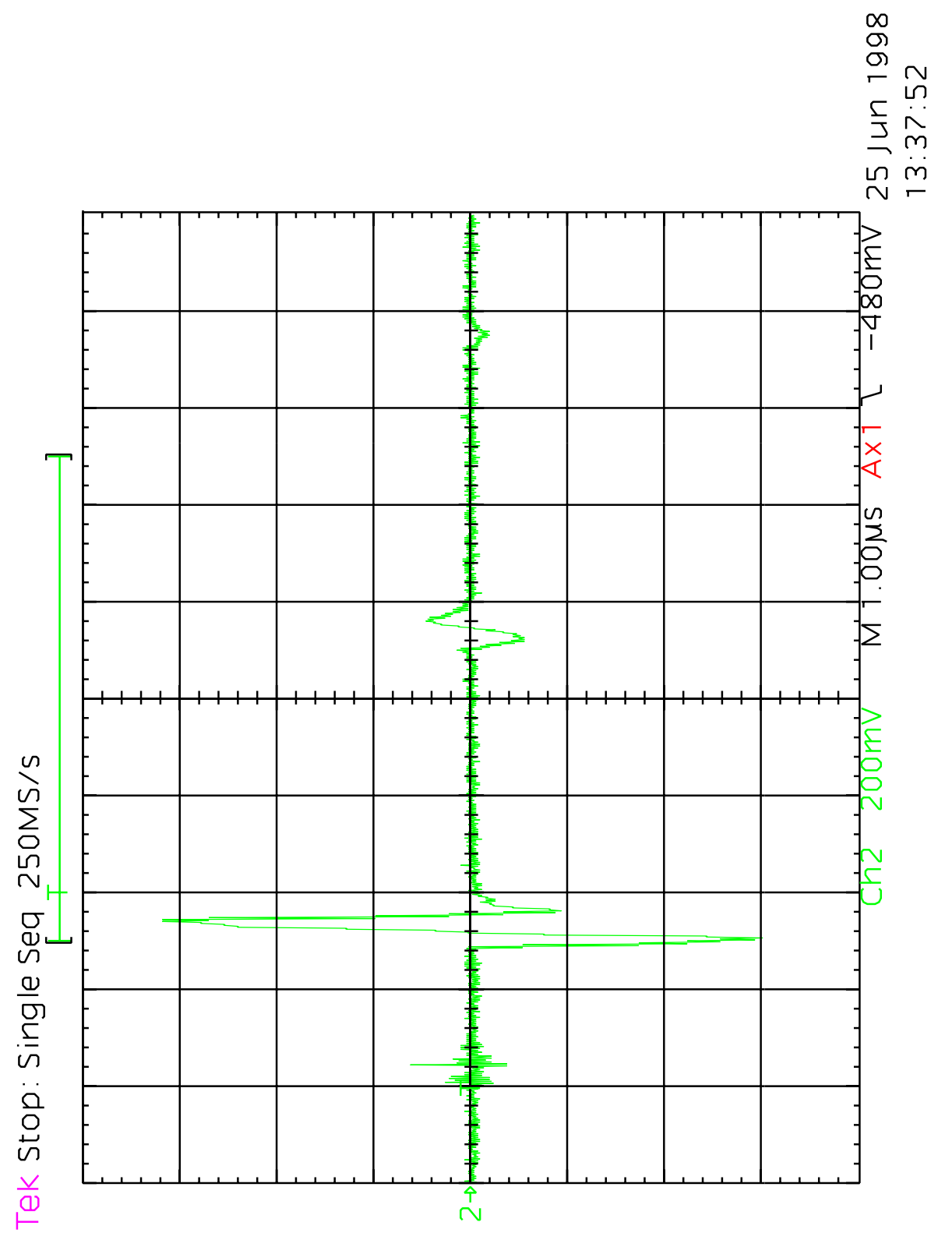

Figure 6.26: Tektronix 620 DSO trace at the pickoff point (T2-T3 junction). This is the signal that provides the timing and cable propagation delay information to the MiniBooNE DAQ system. 
the resistance seen by that particular frequency of current is frequency dependent. This leads to dispersion of a pulse propagating along the cable. The effect is reduced by making the center conductor larger. That is why larger cable, such as RG-8 (a.k.a, RG-213) has better fast-pulse characteristics than a small cable, such as RG-174. There are additional contributions to the dispersion and loss from the dielectric and resistance skin effect in the outer conductor, but these are usually small when compared to the effect of the inner conductor. 


\section{Chapter 7}

\section{Safety Considerations}

\subsection{Fermilab ES\&H Requirements}

We will follow the Fermilab ES\&H Manual (FESHM), which delineates laboratory policy regarding personnel and line management responsibilities and provides technical standards for control of hazards which are peculiar to Fermilab. As discussed in chapter 2010 of the FESHM, MiniBooNE will draft a Safety Assessment Document (SAD) and adhere to the requirements of that chapter.

In addition, the MiniBooNE collaboration will comply with the Particle Physics Division "Procedures for Experiments" (RD-2) for identifying and characterizing hazards in the experiment and reviewing the engineered and administrative controls developed to mitigate these hazards. Experimenter and Spokesperson responsibilities during the life cycle of the experiment are also specified.

The cost and schedule implications of all ES\&H systems and protocols required by the experiment are included in the MiniBooNE Cost and Schedule Plan.

\subsection{Description of Hazards}

The MiniBooNE detector presents a small set of well defined personnel hazards and a negligible environmental hazard. Common workplace hazards such as working at elevated heights are addressed by adherence to OSHA, NEC, and NFPA. Other unusual hazards and their associated controls are summarized in the following sections. 


\subsubsection{Mechanical Hazards}

The most significant mechanical hazard concerns the scaffolding required for phototube mounting inside the tank. The scaffolding is described in chapter 4 . All mechanical systems comply with OSHA.

\subsubsection{Electrical Hazards}

There are numerous high and low voltage supplies which power the phototubes and readout electronics. These are commercial supplies that are matched appropriately to the rating of the power distribution systems. All electrical systems comply with NEC and FESHM.

\subsubsection{Fire Hazards}

The 807 tons of mineral oil in the detector tank poses a possible fire hazard. This is largely mitigated by the high flash point $\left(>340^{\circ} \mathrm{F}\right)$ of oil and by having the oil in a nitrogen environment. The MiniBooNE enclosures are provided with fire detection.

\subsubsection{Laser Hazards}

A pulsed laser will be used for calibration and monitoring. The optical paths are shielded to prevent accidental exposure. Also, access to the laser requires adherence to laser lock-out tag-out procedures mandated by the FESHM.

\subsection{Summary}

Physical hazards associated with the MiniBooNE detector are similar to those encountered in an industrial setting. Administrative controls are defined to ensure that the detector is constructed and operated safely. The only conceivable environmental hazard would be airborne hydrocarbons in the unlikely event of a fire. 


\section{Bibliography}

[1] E. Church et al., "A proposal for an experiment to measure $\nu_{\mu} \rightarrow \nu_{e}$ oscillations and $\nu_{\mu}$ disappearance at the Fermilab Booster: BooNE", LA-UR98-352, Fermilab experiment 898.

[2] C. Athanassopoulos et al. , Phys. Rev. Lett. 75, 2650 (1995); C. Athanassopoulos et al. , Phys. Rev. Lett. 77, 3082 (1996); C. Athanassopoulos et al. , Phys. Rev. C. 54, 2685 (1996); C. Athanassopoulos et al., Phys. Rev. Lett. 81, 1774 (1998); C. Athanassopoulos et al. , Phys. Rev. C. 58, 2489 (1998).

[3] R. Becker-Szendy et al. , Phys. Rev. D46, 3720 (1992).

[4] K. S. Hirata et al. , Phys. Lett. B280, 146 (1992); Y. Fukuda et al. , Phys. Lett. B335, 237 (1994).

[5] Y. Fukuda et al., Phys. Rev. Lett. 81, 1562 (1998).

[6] C. Athanassopoulos et al., NIM A388, 149 (1997).

[7] Hamamatsu Photonics K.K., Electron Tube Division, 314-5, Shimokanzo, Toyooka-village, Iwata-gun, Shizuoka-ken, 438-01 Japan; US sales office: Hamamatsu Corporation, 360 Foothill Road, P. O. Box 6910, Bridgewater, N. J. 08807-1910 Telephone (908) 231-0960.

[8] These results are based on early test results of the MILAGRO collaboration, which has purchased approximately 800 R5912s. The Milagro tubes were not Hamamatsu's standard production models; instead they were "seconds" and do not represent the full quality of the current R5912. Private communication, 1996. 